\title{
Canonical Forms of $3 \times 3$ Strongly and Nonstrictly Hyperbolic Systems with Complex Constant Coefficients
}

\author{
By \\ Yorimasa Oshime*
}

\section{§1. Introduction}

Consider an $m \times m$ system of differential equations

$$
\frac{\partial u}{\partial t}=\sum_{i=1}^{n} A_{i} \frac{\partial u}{\partial x_{i}}
$$

where $u$ is an $m$-vector and $A_{i}$ are complex constant $m \times m$ matrix coefficients. Here the independent variables $x_{i}(i=1, \ldots, n)$ and $t$ are real.

It was Yamaguti and Kasahara [4], [9] who gave the definition and a criterion for the system (1.1) to be strongly hyperbolic (see Theorem 2.4 below for their criterion). Later, Strang [8] proved that (1.1) is strongly hyperbolic if and only if its initial value problem is $L^{2}$-wellposed. However, few attempts have been made to find out all the canonical forms of strongly hyperbolic systems (1.1). It is perhaps because the criterion of Yamaguti and Kasahara is stated in terms of the linear combinations of $A_{1}, A_{2}, \ldots, A_{n}$ and seems difficult to verify directly. The only exception is the case of $m=2(2 \times 2$ systems). In fact, Strang [8] proved that every strongly $2 \times 2$ system can be reduced to a symmetric system (see Definition 2.5). However, the case $m \geq 3$ is much more delicate.

In a previous paper [6], the present author classified the strongly hyperbolic $3 \times 3$ systems with real constant coefficients, using the above mentioned criterion of Yamaguti and Kasahara (see also [7]). The purpose of this paper is to study the same problem for the $3 \times 3$ systems with complex constant coefficients, limiting ourselves to nonstrictly hyperbolic systems (see Definition 2.6 below).

Communicated by S. Matsuura, August 7, 1990. Revised July 4, 1991.

1991 Mathematics Subject Classifications: 35 L40

* Junior College of Economics, Wakayama University, Wakayama, 640.

Present address: Faculty of Engineering, Doshisha University, Kyoto, 602, Japan. 
All the results of this paper shall be summarized in the last section in terms of matrix families.

\section{§2. Definitions}

Throughout this paper, we consider only complex square (actually $3 \times 3$ ) matrices and their linear combinations with real coefficients. We usually denote real constants by lower case greek letters, complex constants by lower case roman letters, matrices by upper case roman letters unless otherwise indicated.

Definition 2.1. Let $A_{j}(j=1,2, \ldots, n)$ be $m \times m$ complex matrices. The set of all their linear combinations

$$
A(\xi)=A\left(\xi_{1}, \xi_{2}, \ldots, \xi_{n}\right)=\sum_{j=1}^{n} \xi_{j} A_{j} \quad \text { with } \quad \xi_{j} \in \mathbb{R}(j=1,2, \ldots, n)
$$

is said to be the matrix family spanned by $A_{1}, A_{2}, \ldots, A_{n}$ and is denoted by $\left\langle A_{1}, A_{2}, \ldots, A_{n}\right\rangle$.

Definition 2.2. A matrix family $\left\langle A_{1}, A_{2}, \ldots, A_{n}\right\rangle$ is called real-diagonalizable if for every $A(\xi) \in\left\langle A_{1}, A_{2}, \ldots, A_{n}\right\rangle$, there exists a matrix $S(\xi)$ (called a diagonalizer) such that

$$
S(\xi)^{-1} A(\xi) S(\xi)
$$

is a real diagonal matrix.

Definition 2.3. A matrix family $\left\langle A_{1}, A_{2}, \ldots, A_{n}\right\rangle$ is called uniformly realdiagonalizable if it is real-diagonalizable and there is a diagonalizer $S(\xi)$ such that there exists a constant $M>0$ independent of $\xi$ for which

$$
\|S(\xi)\|,\left\|S(\xi)^{-1}\right\| \leq M
$$

when $\xi$ runs over $\mathbb{R}^{n}$. Similarly, a matrix family is called non-uniformly realdiagonalizable if any diagonalizer is unbounded when $\xi$ runs over $\mathbb{R}^{n}$.

We quote here the most fundamental theorem concerning the equation (1.1).

Theorem 2.4 (Yamaguti-Kasahara [9]). Equation (1.1) is strongly hyperbolic if and only if the matrix family $\left\langle A_{1}, A_{2}, \ldots, A_{n}\right\rangle$ is uniformly realdiagonalizable.

Remark. As mentioned in Introduction, strong hyperbolicity is equivalent to $L^{2}$-wellposedness in the case of constant coefficients. For the proof of Theorem 2.4, see Yamaguti-Kasahara [9], Kasahara-Yamaguti [4] ( $B^{\infty}$-theory), or Strang $[8]\left(\mathbb{L}^{2}\right.$-theory).

We now introduce the most important subclass of the real-diagonalizable matrix families. 
Definition 2.5. A matrix family $\left\langle A_{1}, A_{2}, \ldots, A_{n}\right\rangle$ is called hermitian if all of $A_{1}, A_{2}, \ldots, A_{n}$ are hermitian. In addition, equation (1.1) with those $A_{j}$ 's is called a symmetric hyperbolic system.

Remark. In this case, as a diagonalizer $S(\xi)$, we can take a unitary matrix depending on $\xi$. Consequently, any hermitian family is uniformly realdiagonalizable.

The following is the very class of the $3 \times 3$ matrix families we shall classify.

Defimition 2.6. A matrix family $\left\langle A_{1}, A_{2}, \ldots, A_{n}\right\rangle$ is said to have multiple eigenvalues if some $A(\xi) \in\left\langle A_{1}, A_{2}, \ldots, A_{n}\right\rangle$ with $\xi \neq(0, \ldots, 0)$ does. In addition, a strongly hyperbolic system (1.1) is said to be strongly and nonstrictly hyperbolic if $\left\langle A_{1}, A_{2}, \ldots, A_{n}\right\rangle$ has multiple eigenvalues.

Let us now consider what kind of equivalence relation should be introduced for matrix families. It is easy to see the following three operations $\left\langle A_{1}, \ldots, A_{n}\right\rangle \rightarrow\left\langle B_{1}, \ldots, B_{n^{\prime}}\right\rangle$ do not affect the real-diagonalizability (uniform or not) of matrix families.

a) Change of basis.

$$
\begin{aligned}
& B_{1}=m_{11} A_{1}+m_{12} A_{2}+\cdots+m_{1 n} A_{n} \\
& B_{2}=m_{21} A_{1}+m_{22} A_{2}+\cdots+m_{2 n} A_{n} \\
& B_{n}=m_{n 1} A_{1}+m_{n 2} A_{2}+\cdots+m_{n n} A_{n}
\end{aligned}
$$

where $M=\left(m_{i j}\right)$ is a real nonsingular $n \times n$ matrix.

b) Addition of scalar multiples of identity.

$$
\begin{array}{cc}
B_{1}=A_{1}+\mu_{1} I \\
B_{2}=A_{2}+\mu_{2} I \\
\vdots & \vdots \\
B_{n}= & A_{n}+\mu_{n} I
\end{array}
$$

where $I$ is the identity matrix and $\mu_{i}(1 \leq i \leq n)$ are reals.

c) Similarity transformation.

$$
\begin{array}{cc}
B_{1}= & T^{-1} A_{1} T \\
B_{2} & =T^{-1} A_{2} T \\
\vdots & \vdots \\
B_{n}= & T^{-1} A_{n} T
\end{array}
$$

where $T$ is some complex nonsingular $m \times m$ matrix arbitrarily fixed. 
Let us consider how the above three operations transform the original differential equation (1.1). First, a) corresponds to a change of the space variables:

$$
\left(\tilde{x}_{1}, \tilde{x}_{2}, \ldots, \tilde{x}_{n}\right)^{\mathbb{T}}=M\left(x_{1}, x_{2}, \ldots, x_{n}\right)^{\mathbb{T}} .
$$

Second, b) corresponds to a change of the time-space variables of the type:

$$
\tilde{x}_{i}=x_{i}-\mu_{i} t \quad(1 \leq i \leq n) .
$$

Note that if some space variables disappear from (1.1) by these operations, they can be regarded as parameters of the initial data for the reduced equation. Finally, c) corresponds to a change of the unknowns:

$$
\left(\tilde{u}_{1}, \tilde{u}_{2}, \ldots, \tilde{u}_{n}\right)^{\mathbb{T}}=T^{-1}\left(u_{1}, u_{2}, \ldots, u_{n}\right)^{\mathbb{T}} .
$$

Combining the above operations a), b) and c), we can define the equivalence relation among matrix families as follows.

Definition 2.7. Matrix families $\left\langle A_{1}, A_{2}, \ldots, A_{n}\right\rangle$ and $\left\langle B_{1}, B_{2}, \ldots, B_{n^{\prime}}\right\rangle$ are called equivalent if there exist a nonsingular matrix $T$ and $\mu_{j} \in \mathbb{R}(j=1,2, \ldots, n)$ such that

$$
\left\langle T^{-1} A_{1} T-\mu_{1} I, T^{-1} A_{2} T-\mu_{2} I, \ldots, T^{-1} A_{n} T-\mu_{n} I\right\rangle=\left\langle B_{1}, B_{2}, \ldots, B_{n^{\prime}}\right\rangle .
$$

And we denote this equivalence relation by

$$
\left\langle A_{1}, A_{2}, \ldots, A_{n}\right\rangle \sim\left\langle B_{1}, B_{2}, \ldots, B_{n^{\prime}}\right\rangle .
$$

By using the above operations a) and b), it is easy to see that any matrix family is equivalent to some $\left\langle B_{1}, \ldots, B_{n}\right\rangle$ where $B_{1}, B_{2}, \ldots, B_{n}$ are linearly independent and none of their nonzero linear combinations is equal to a scalar multiple of identity. Let us define a word indicating this property for later convenience.

Definition 2.8. A matrix family $\left\langle A_{1}, A_{2}, \ldots, A_{n}\right\rangle$ is called nondegenerate if $I, A_{1}, A_{2}, \ldots, A_{n}$ are linearly independent over the field of real numbers.

Note that the definitions in this section are valid for the square matrices of an arbitrary size, although we limit ourselves to study $3 \times 3$ matrix families which are uniformly or non-uniformly real-diagonalizable. And we shall treat the problem purely as that in the matrix theory and shall not refer to the differential equation (1.1) any more.

\section{§3. Preliminaries}

In this paper, we study exclusively real-diagonalizable $3 \times 3$ matrix families such that at least one of their nonzero members has a multiple eigenvalue. For such a family $\left\langle A_{1}, A_{2}, \ldots, A_{n}\right\rangle$, changing the basis if necessary, we may assume 
$A_{1}$ has a multiple (real) eigenvalue. If this multiple eigenvalue is triple, the $3 \times 3$ matrix $A_{1}$ must be a real multiple of identity and we may ignore this case (see Definition 2.7). So we may assume $A_{1}$ has a double eigenvalue. Multiplying $A_{1}$ by a suitable real and adding to it a suitable real scalar multiple of identity, we may assume the eigenvalues of $A_{1}$ are $1,0,0$. Hence, by use of the similarity transformation diagonalizing $A_{1}$, we may further assume

$$
A_{1}=\left[\begin{array}{lll}
1 & 0 & 0 \\
0 & 0 & 0 \\
0 & 0 & 0
\end{array}\right] \text {. }
$$

To investigate the property of an arbitrary $B \in\left\langle A_{1}, A_{2}, \ldots, A_{n}\right\rangle$, let us quote the following lemma from [7].

Lemma 3.1. Let $f(\lambda, \xi)$ be a cubic polynomial of the form

$$
f(\lambda, \xi) \equiv \lambda^{3}+a_{1} \lambda^{2}+a_{2} \lambda+a_{3}+\xi\left(b_{0} \lambda^{2}+b_{1} \lambda+b_{2}\right)
$$

where $a_{1}, a_{2}, a_{3}, b_{0} \neq 0, b_{1}, b_{2}$ are real constants and $\xi$ is a real parameter. Then the cubic equation

$$
f(\lambda, \xi)=0
$$

has only real roots for any $\xi \in \mathbb{R}$ if and only if

$$
\lambda^{3}+a_{1} \lambda^{2}+a_{2} \lambda+a_{3}=0
$$

and

$$
b_{0} \lambda^{2}+b_{1} \lambda+b_{2}=0
$$

have only real roots, say, $\alpha_{1} \leq \alpha_{2} \leq \alpha_{3}$ for the first equation and $\beta_{1} \leq \beta_{2}$ for the second, and the inequality

$$
\alpha_{1} \leq \beta_{1} \leq \alpha_{2} \leq \beta_{2} \leq \alpha_{3}
$$

holds.

The following lemma will be also useful in the sequel.

Lemma 3.2. Let $\sigma_{j}(\eta), \tilde{\sigma}_{j}(\eta), \sigma_{k}(\eta), \tilde{\sigma}_{k}(\eta)$ be polynomials in $\eta=\left(\eta_{1}, \eta_{2}, \ldots, \eta_{n}\right)$ with real coefficients. Suppose that either

$$
\left\{\sigma_{j}(\eta)+i \tilde{\sigma}_{j}(\eta)\right\}\left\{\sigma_{k}(\eta)+i \tilde{\sigma}_{k}(\eta)\right\} \quad(i=\sqrt{-1})
$$

is real and positive or

$$
\sigma_{j}(\eta)+i \tilde{\sigma}_{j}(\eta)=\sigma_{k}(\eta)+i \tilde{\sigma}_{k}(\eta)=0
$$

holds for any choice of $\eta \in \mathbb{R}^{n}$. Then there exist polynomials $\mu(\eta), \tilde{\mu}(\eta), \varphi(\eta)$, $\psi(\eta)$ with real coefficients which satisfy 


$$
\begin{aligned}
\sigma_{j}(\eta) \equiv \mu(\eta) \varphi(\eta), & & \tilde{\sigma}_{j}(\eta) \equiv \tilde{\mu}(\eta) \varphi(\eta) \\
\sigma_{k}(\eta) \equiv \mu(\eta) \psi(\eta), & & \tilde{\sigma}_{k}(\eta) \equiv-\tilde{\mu}(\eta) \psi(\eta)
\end{aligned}
$$

and

1) $\mu(\eta)$ and $\tilde{\mu}(\eta)$ have no common factors other than nonzero constants,

2) $\operatorname{sgn} \varphi(\eta)=\operatorname{sgn} \psi(\eta)$ for all $\eta \in \mathbb{R}^{n}$ unless $\mu(\eta)=\tilde{\mu}(\eta)=0$. Especially,

$$
\begin{aligned}
& \operatorname{sgn} \sigma_{j}(\eta)=\operatorname{sgn} \sigma_{k}(\eta), \\
& \operatorname{sgn} \tilde{\sigma}_{j}(\eta)=-\operatorname{sgn} \tilde{\sigma}_{k}(\eta)
\end{aligned}
$$

hold for all $\eta \in \mathbb{R}^{n}$.

Proof. Because the imaginary part of

$$
\left\{\sigma_{j}(\eta)+i \tilde{\sigma}_{j}(\eta)\right\}\left\{\sigma_{k}(\eta)+i \tilde{\sigma}_{k}(\eta)\right\}
$$

always vanishes for any $\eta \in \mathbb{R}^{n}$, we have

$$
\sigma_{j}(\eta) \tilde{\sigma}_{k}(\eta) \equiv-\sigma_{k}(\eta) \tilde{\sigma}_{j}(\eta)
$$

We may assume that neither $\sigma_{j}(\eta)$ nor $\tilde{\sigma}_{j}(\eta)$ is identically zero because otherwise we have $\sigma_{j}(\eta) \equiv \tilde{\sigma}_{j}(\eta) \equiv 0, \sigma_{j}(\eta) \equiv \sigma_{k}(\eta) \equiv 0$ or $\tilde{\sigma}_{j}(\eta) \equiv \tilde{\sigma}_{k}(\eta) \equiv 0$ and the conclusion is immediate. Put

$$
\sigma_{j}(\eta) \equiv \mu(\eta) \varphi(\eta), \quad \tilde{\sigma}_{j}(\eta) \equiv \tilde{\mu}(\eta) \varphi(\eta),
$$

where $\mu(\eta)$ and $\tilde{\mu}(\eta)$ have no common factors. So (3.6) implies

$$
\mu(\eta) \tilde{\sigma}_{k}(\eta) \equiv-\tilde{\mu}(\eta) \sigma_{k}(\eta)
$$

Because $\mu(\eta)$ and $\tilde{\mu}(\eta)$ have no common factors, we further obtain

$$
\sigma_{k}(\eta) \equiv \mu(\eta) \psi(\eta), \quad \tilde{\sigma}_{k}(\eta) \equiv-\tilde{\mu}(\eta) \psi(\eta)
$$

where $\psi(\eta)$ is a certain polynomial with real coefficients. By (3.7) and (3.8), the assumption of the lemma becomes as follows: Either

$$
\left\{\sigma_{j}(\eta)+i \tilde{\sigma}_{j}(\eta)\right\}\left\{\sigma_{k}(\eta)+i \tilde{\sigma}_{k}(\eta)\right\} \equiv \varphi(\eta) \psi(\eta)\left\{(\mu(\eta))^{2}+(\tilde{\mu}(\eta))^{2}\right\}
$$

is positive or

$$
\sigma_{j}(\eta)+i \tilde{\sigma}_{j}(\eta) \equiv \varphi(\eta)\{\mu(\eta)+i \tilde{\mu}(\eta)\}=0
$$

and

$$
\sigma_{k}(\eta)+i \tilde{\sigma}_{k}(\eta) \equiv \psi(\eta)\{\mu(\eta)+i \tilde{\mu}(\eta)\}=0
$$

hold simultaneously, for any choice of $\eta \in \mathbb{R}^{n}$. This fact implies

$$
\operatorname{sgn} \varphi(\eta)=\operatorname{sgn} \psi(\eta)
$$


unless

$$
\mu(\eta)=\tilde{\mu}(\eta)=0
$$

Thus the proof is complete.

Applying Lemma 3.1 to the characteristic equation of $\xi A_{1}+B$, we have the following lemma.

Lemma 3.3. Suppose that $\left\langle A_{1}, A_{2}, \ldots, A_{n}\right\rangle$ is a real-diagonalizable family with

$$
A_{1}=\left[\begin{array}{lll}
1 & 0 & 0 \\
0 & 0 & 0 \\
0 & 0 & 0
\end{array}\right]
$$

Suppose also that

$$
B=\left[\begin{array}{lll}
b_{11} & b_{12} & b_{22} \\
b_{21} & b_{22} & b_{23} \\
b_{31} & b_{32} & b_{33}
\end{array}\right]
$$

is an arbitrary member of $\left\langle A_{1}, A_{2}, \ldots, A_{n}\right\rangle$. Then the right-lower submatrix of $B$ :

$$
\left[\begin{array}{ll}
b_{22} & b_{23} \\
b_{32} & b_{33}
\end{array}\right]
$$

has only real eigenvalues and $b_{11}$ is real.

Proof. Because $\xi A_{1}+B \in\left\langle A_{1}, A_{2}, \ldots, A_{n}\right\rangle$ is real-diagonalizable and has only real eigenvalues, its characteristic equation turns out to be

$$
\begin{aligned}
\operatorname{det}\left(-\lambda I+\xi A_{1}+B\right) & \equiv \operatorname{det}\left[\begin{array}{ccc}
-\lambda+\xi+b_{11} & b_{12} & b_{22} \\
b_{21} & -\lambda+b_{22} & b_{23} \\
b_{31} & b_{32} & -\lambda+b_{33}
\end{array}\right] \\
& \equiv \operatorname{det}(-\lambda I+B)+\xi \operatorname{det}\left[\begin{array}{cc}
-\lambda+b_{22} & b_{23} \\
b_{32} & -\lambda+b_{33}
\end{array}\right]=0
\end{aligned}
$$

Therefore, from Lemma 3.1, we know that

$$
\left[\begin{array}{ll}
b_{22} & b_{23} \\
b_{32} & b_{33}
\end{array}\right]
$$

has only real eigenvalues. Consequently, its trace

$$
b_{22}+b_{33}
$$

is real. On the other hand, the real-diagonalizability of $B$ assures that its trace 


$$
b_{11}+b_{22}+b_{33}
$$

is also real. Thus $b_{11}$ is real as the difference of these two real numbers.

It is clear that the right-lower $2 \times 2$ submatrices of the members of $\left\langle A_{1}, A_{2}, \ldots, A_{n}\right\rangle$ form a $2 \times 2$ matrix family. Thus the above Lemma 3.3 asserts that this $2 \times 2$ matrix family has only real eigenvalues. Now we can proceed just in the same way as in Strang [8] and conclude that the $2 \times 2$ matrix family is equivalent to a hermitian family or an upper-triangular family. Using another similarity transformation with a diagonal matrix, if necessary, this $2 \times 2$ matrix family is reduced to one of the following 1), 2), $\ldots, 8)$.
1) $\left\langle\left[\begin{array}{ll}0 & 0 \\ 0 & 0\end{array}\right]\right\rangle$.
2) $\left\langle\left[\begin{array}{rr}1 & 0 \\ 0 & -1\end{array}\right]\right\rangle$.
3) $\left\langle\left[\begin{array}{rr}1 & 0 \\ 0 & -1\end{array}\right],\left[\begin{array}{ll}0 & 1 \\ 1 & 0\end{array}\right]\right\rangle$.
4) $\left\langle\left[\begin{array}{rr}1 & 0 \\ 0 & -1\end{array}\right],\left[\begin{array}{ll}0 & 1 \\ 1 & 0\end{array}\right],\left[\begin{array}{rr}0 & -i \\ i & 0\end{array}\right]\right\rangle$.
5) $\left\langle\left[\begin{array}{ll}0 & 1 \\ 0 & 0\end{array}\right]\right\rangle$.
6) $\left\langle\left[\begin{array}{ll}0 & 1 \\ 0 & 0\end{array}\right],\left[\begin{array}{ll}0 & i \\ 0 & 0\end{array}\right]\right\rangle$.
7) $\left\langle\left[\begin{array}{rr}1 & 0 \\ 0 & -1\end{array}\right],\left[\begin{array}{ll}0 & 1 \\ 0 & 0\end{array}\right]\right\rangle$.
8) $\left\langle\left[\begin{array}{rr}1 & 0 \\ 0 & -1\end{array}\right],\left[\begin{array}{ll}0 & 1 \\ 0 & 0\end{array}\right],\left[\begin{array}{ll}0 & i \\ 0 & 0\end{array}\right]\right\rangle$.

Let us denote by $\tilde{T}$ the transformation (in the form of $2 \times 2$ matrix ) for this reduction. Now let us turn to the $3 \times 3$ matrix family. Note that the similarity transformation with

$$
T=\left[\begin{array}{ll}
1 & 0 \\
0 & \tilde{T}
\end{array}\right],
$$

and some change of basis and a certain addition of real multiples of identities reduce the right-lower submatrix family to the above 1$), 2), \ldots, 8$ ). Note also that the similarity transformation with $\tilde{T}$ does not affect the real $(1,1)$-entry of each $A(\xi) \in\left\langle A_{1}, A_{2}, \ldots, A_{n}\right\rangle$. Thus the $3 \times 3$ matrix family can be reduced to one of the following 1$), 2), \ldots, 8)$. 
1) $\left.\left\langle\left[\begin{array}{lll}1 & 0 & 0 \\ 0 & 0 & 0 \\ 0 & 0 & 0\end{array}\right],\left[\begin{array}{ccc}0 & b_{1} & b_{2} \\ b_{3} & 0 & 0 \\ b_{4} & 0 & 0\end{array}\right], \ldots\right\rangle\right\rangle$

2) $\left.\left\langle\left[\begin{array}{lll}1 & 0 & 0 \\ 0 & 0 & 0 \\ 0 & 0 & 0\end{array}\right],\left[\begin{array}{ccc}0 & b_{1} & b_{2} \\ b_{3} & 1 & 0 \\ b_{4} & 0 & -1\end{array}\right],\left[\begin{array}{ccc}0 & c_{1} & c_{2} \\ c_{3} & 0 & 0 \\ c_{4} & 0 & 0\end{array}\right], \ldots\right\rangle\right\rangle$,

3) $\left.\left\langle\left[\begin{array}{lll}1 & 0 & 0 \\ 0 & 0 & 0 \\ 0 & 0 & 0\end{array}\right],\left[\begin{array}{ccc}0 & b_{1} & b_{2} \\ b_{3} & 1 & 0 \\ b_{4} & 0 & -1\end{array}\right],\left[\begin{array}{ccc}0 & c_{1} & c_{2} \\ c_{3} & 0 & 1 \\ c_{4} & 1 & 0\end{array}\right],\left[\begin{array}{ccc}0 & d_{1} & d_{2} \\ d_{3} & 0 & 0 \\ d_{4} & 0 & 0\end{array}\right], \ldots\right\rangle\right\rangle$,

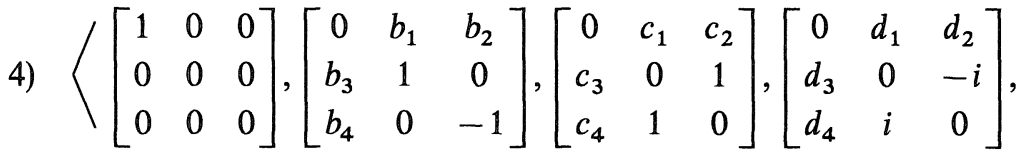

$$
\left.\left[\begin{array}{ccc}
0 & e_{1} & e_{2} \\
e_{3} & 0 & 0 \\
e_{4} & 0 & 0
\end{array}\right], \ldots\right\rangle
$$

5) $\left.\left\langle\left[\begin{array}{lll}1 & 0 & 0 \\ 0 & 0 & 0 \\ 0 & 0 & 0\end{array}\right],\left[\begin{array}{ccc}0 & b_{1} & b_{2} \\ b_{3} & 0 & 1 \\ b_{4} & 0 & 0\end{array}\right],\left[\begin{array}{ccc}0 & c_{1} & c_{2} \\ c_{3} & 0 & 0 \\ c_{4} & 0 & 0\end{array}\right], \ldots\right\rangle\right\rangle$,

6) $\left.\left\langle\left[\begin{array}{lll}1 & 0 & 0 \\ 0 & 0 & 0 \\ 0 & 0 & 0\end{array}\right],\left[\begin{array}{ccc}0 & b_{1} & b_{2} \\ b_{3} & 0 & 1 \\ b_{4} & 0 & 0\end{array}\right],\left[\begin{array}{ccc}0 & c_{1} & c_{2} \\ c_{3} & 0 & i \\ c_{4} & 0 & 0\end{array}\right],\left[\begin{array}{ccc}0 & d_{1} & d_{2} \\ d_{3} & 0 & 0 \\ d_{4} & 0 & 0\end{array}\right], \ldots\right\rangle\right\rangle$,

7) $\left.\left\langle\left[\begin{array}{lll}1 & 0 & 0 \\ 0 & 0 & 0 \\ 0 & 0 & 0\end{array}\right],\left[\begin{array}{ccc}0 & b_{1} & b_{2} \\ b_{3} & 1 & 0 \\ b_{4} & 0 & -1\end{array}\right],\left[\begin{array}{ccc}0 & c_{1} & c_{2} \\ c_{3} & 0 & 1 \\ c_{4} & 0 & 0\end{array}\right],\left[\begin{array}{ccc}0 & d_{1} & d_{2} \\ d_{3} & 0 & 0 \\ d_{4} & 0 & 0\end{array}\right], \ldots\right\rangle\right\rangle$,

8) $\left\langle\left[\begin{array}{lll}1 & 0 & 0 \\ 0 & 0 & 0 \\ 0 & 0 & 0\end{array}\right],\left[\begin{array}{ccc}0 & b_{1} & b_{2} \\ b_{3} & 1 & 0 \\ b_{4} & 0 & -1\end{array}\right],\left[\begin{array}{ccc}0 & c_{1} & c_{2} \\ c_{3} & 0 & 1 \\ c_{4} & 0 & 0\end{array}\right],\left[\begin{array}{ccc}0 & d_{1} & d_{2} \\ d_{3} & 0 & i \\ d_{4} & 0 & 0\end{array}\right]\right.$,

$$
\left.\left[\begin{array}{ccc}
0 & e_{1} & e_{2} \\
e_{3} & 0 & 0 \\
e_{4} & 0 & 0
\end{array}\right], \ldots\right\rangle
$$

where $b_{1}, b_{2}, \ldots, e_{3}, e_{4}$ are certain complex constants. We shall consider each case separately in the sequel. 


\section{\$4. Families Spanned by Two Matrices}

The discussions in this section is almost the same as that in Section 3 of [6]. So we shall omit most of the proofs.

Proposition 4.1. Put

$$
A=\left[\begin{array}{lll}
1 & 0 & 0 \\
0 & 0 & 0 \\
0 & 0 & 0
\end{array}\right], \quad B=\left[\begin{array}{ccc}
0 & b_{1} & b_{2} \\
b_{3} & 0 & 0 \\
b_{4} & 0 & 0
\end{array}\right] \neq 0
$$

where $b_{j}(j=1, \ldots, 4)$ are complex constants. Then the matrix family $\langle A, B\rangle$ spanned by the above $A, B$ is real-diagonalizable if and only if $b_{1} b_{3}+b_{2} b_{4}$ is real and positive:

$$
b_{1} b_{3}+b_{2} b_{4}>0
$$

Proof. The same argument as in Proposition 3.2 of [6] is valid if we take some care about the complexity of $b_{j}$. So we omit the detail.

Proposition 4 .2. Let the matrix family $\langle A, B\rangle$ spanned by

$$
A=\left[\begin{array}{lll}
1 & 0 & 0 \\
0 & 0 & 0 \\
0 & 0 & 0
\end{array}\right], \quad B=\left[\begin{array}{ccc}
0 & b_{1} & b_{2} \\
b_{3} & 0 & 0 \\
b_{4} & 0 & 0
\end{array}\right] \neq 0
$$

be real-diagonalizable. Then there exists a certain nonsingular $T$ such that $T^{-1} A T$ and $T^{-1} B T$ are simultaneously hermitian. Moreover

$$
T^{-1} A T=\left[\begin{array}{lll}
1 & 0 & 0 \\
0 & 0 & 0 \\
0 & 0 & 0
\end{array}\right], \quad T^{-1} B T=\alpha\left[\begin{array}{lll}
0 & 1 & 0 \\
1 & 0 & 0 \\
0 & 0 & 0
\end{array}\right]
$$

Here $\alpha>0$ is some real constant.

Proof. From Proposition 4.1, we have

$$
b_{1} b_{3}+b_{2} b_{4}>0 \text {. }
$$

By putting

$$
\begin{aligned}
& \alpha=\sqrt{b_{1} b_{3}+b_{2} b_{4}}, \\
& \mathbb{T}=\left[\begin{array}{ccc}
1 & 0 & 0 \\
0 & b_{3} / \alpha & -b_{2} / \alpha \\
0 & b_{4} / \alpha & b_{1} / \alpha
\end{array}\right],
\end{aligned}
$$

we obtain the conclusion. 
Proposition 4.3. Put

$$
A=\left[\begin{array}{lll}
1 & 0 & 0 \\
0 & 0 & 0 \\
0 & 0 & 0
\end{array}\right], \quad B=\left[\begin{array}{ccc}
0 & b_{1} & b_{2} \\
b_{3} & 1 & 0 \\
b_{4} & 0 & -1
\end{array}\right]
$$

where $b_{j}(j=1, \ldots, 4)$ are complex constants. Then the matrix family $\langle A, B\rangle$ spanned by the above $A, B$ is real-diagonalizable if and only if both of the following 1) and 2) are satisfied.

1) $b_{1} b_{3}>0$ or $b_{1}=b_{3}=0$.

2) $b_{2} b_{4}>0$ or $b_{2}=b_{4}=0$.

Proof. The same argument as in Proposition 3.4 of [6] is valid if we take some care about the complexity of $b_{j}$. So we omit the detail again.

Proposition 4.4. Let the matrix family $\langle A, B\rangle$ spanned by

$$
A=\left[\begin{array}{lll}
1 & 0 & 0 \\
0 & 0 & 0 \\
0 & 0 & 0
\end{array}\right], \quad B=\left[\begin{array}{ccc}
0 & b_{1} & b_{2} \\
b_{3} & 1 & 0 \\
b_{4} & 0 & -1
\end{array}\right]
$$

be real-diagonalizable. Then it is simultaneously symmetrized by some $T$ as follows.

$$
T^{-1} A T=\left[\begin{array}{lll}
1 & 0 & 0 \\
0 & 0 & 0 \\
0 & 0 & 0
\end{array}\right], \quad T^{-1} B T=\left[\begin{array}{rrr}
0 & \alpha & \beta \\
\alpha & 1 & 0 \\
\beta & 0 & -1
\end{array}\right]
$$

where $\alpha, \beta$ are some real constants.

Proof. From Proposition 4.3, we have

$$
b_{1} b_{3}>0 \quad \text { or } \quad b_{1}=b_{3}=0
$$

and

$$
b_{2} b_{4}>0 \quad \text { or } \quad b_{2}=b_{4}=0 \text {. }
$$

By putting

$$
\begin{gathered}
\alpha=\sqrt{b_{1} b_{3}}, \quad \beta=\sqrt{b_{2} b_{4}}, \\
u \begin{cases}=\alpha / b_{1} & \left(\text { if } b_{1} b_{3}>0\right) \\
=1 & \text { (if } \left.b_{1}=b_{3}=0\right)\end{cases} \\
v \begin{cases}=\beta / b_{2} & \text { (if } \left.b_{2} b_{4}>0\right) \\
=1 & \text { (if } \left.b_{2}=b_{4}=0\right)\end{cases}
\end{gathered}
$$




$$
T=\left[\begin{array}{lll}
1 & 0 & 0 \\
0 & u & 0 \\
0 & 0 & v
\end{array}\right]
$$

we have the desired result.

Let us now consider the case where the $2 \times 2$ submatrix family contains a non-diagonalizable member.

Proposition 4.5. Put

$$
A=\left[\begin{array}{lll}
1 & 0 & 0 \\
0 & 0 & 0 \\
0 & 0 & 0
\end{array}\right], \quad B=\left[\begin{array}{ccc}
0 & b_{1} & b_{2} \\
b_{3} & 0 & b_{5} \\
b_{4} & 0 & 0
\end{array}\right] \quad\left(b_{5} \neq 0\right)
$$

where $b_{j}(j=1, \ldots, 5)$ are complex constants. Then the matrix family $\langle A, B\rangle$ is real-diagonalizable if and only if one of the following holds.

1) $b_{1} b_{3}>0$ and $b_{4}=0$.

2) $b_{2} b_{4}>0$ and $b_{1}=0$.

Proof. The same argument as in Proposition 3.6 of [6] is valid if we take some care about the complexity of $b_{j}$. So we omit the proof.

Proposition 4.6. The following holds.

1) Let $b_{1} b_{3}>0$ and $b_{5} \neq 0$. Then

$$
\left\langle\left[\begin{array}{lll}
1 & 0 & 0 \\
0 & 0 & 0 \\
0 & 0 & 0
\end{array}\right],\left[\begin{array}{ccc}
0 & b_{1} & b_{2} \\
b_{3} & 0 & b_{5} \\
0 & 0 & 0
\end{array}\right]\right\rangle \sim\left\langle\left[\begin{array}{lll}
1 & 0 & 0 \\
0 & 0 & 0 \\
0 & 0 & 0
\end{array}\right],\left[\begin{array}{lll}
0 & 1 & 0 \\
1 & 0 & 1 \\
0 & 0 & 0
\end{array}\right]\right\rangle
$$

2) Let $b_{2} b_{4}>0$ and $b_{5} \neq 0$. Then

$$
\left\langle\left[\begin{array}{lll}
1 & 0 & 0 \\
0 & 0 & 0 \\
0 & 0 & 0
\end{array}\right],\left[\begin{array}{ccc}
0 & 0 & b_{2} \\
b_{3} & 0 & b_{5} \\
b_{4} & 0 & 0
\end{array}\right]\right\rangle \sim\left\langle\left[\begin{array}{lll}
1 & 0 & 0 \\
0 & 0 & 0 \\
0 & 0 & 0
\end{array}\right],\left[\begin{array}{lll}
0 & 1 & 0 \\
1 & 0 & 0 \\
0 & 1 & 0
\end{array}\right]\right\rangle
$$

And both matrix families are non-uniformly real-diagonalizable.

Proof. We begin with 1). By putting

$$
\begin{aligned}
\alpha & =\sqrt{b_{1} b_{3}}, \\
T & =\left[\begin{array}{ccc}
1 / b_{3} & 0 & 0 \\
0 & 1 / \alpha & -b_{2} / b_{1} b_{5} \\
0 & 0 & 1 / b_{5}
\end{array}\right],
\end{aligned}
$$

we obtain 


$$
\begin{gathered}
T^{-1}\left[\begin{array}{lll}
1 & 0 & 0 \\
0 & 0 & 0 \\
0 & 0 & 0
\end{array}\right] T=\left[\begin{array}{lll}
1 & 0 & 0 \\
0 & 0 & 0 \\
0 & 0 & 0
\end{array}\right], \\
T^{-1}\left[\begin{array}{ccc}
0 & b_{1} & b_{2} \\
b_{3} & 0 & b_{5} \\
0 & 0 & 0
\end{array}\right] T=\alpha\left[\begin{array}{lll}
0 & 1 & 0 \\
1 & 0 & 1 \\
0 & 0 & 0
\end{array}\right] .
\end{gathered}
$$

On the other hand, the case 2) can be reduced to the transpose of 1) because

$$
\begin{gathered}
{\left[\begin{array}{lll}
1 & 0 & 0 \\
0 & 0 & 1 \\
0 & 1 & 0
\end{array}\right]^{-1}\left[\begin{array}{lll}
1 & 0 & 0 \\
0 & 0 & 0 \\
0 & 0 & 0
\end{array}\right]\left[\begin{array}{lll}
1 & 0 & 0 \\
0 & 0 & 1 \\
0 & 1 & 0
\end{array}\right]=\left[\begin{array}{lll}
1 & 0 & 0 \\
0 & 0 & 0 \\
0 & 0 & 0
\end{array}\right]} \\
{\left[\begin{array}{lll}
1 & 0 & 0 \\
0 & 0 & 1 \\
0 & 1 & 0
\end{array}\right]^{-1}\left[\begin{array}{ccc}
0 & 0 & b_{2} \\
b_{3} & 0 & b_{5} \\
b_{4} & 0 & 0
\end{array}\right]\left[\begin{array}{lll}
1 & 0 & 0 \\
0 & 0 & 1 \\
0 & 1 & 0
\end{array}\right]=\left[\begin{array}{ccc}
0 & b_{2} & 0 \\
b_{4} & 0 & 0 \\
b_{3} & b_{5} & 0
\end{array}\right] .}
\end{gathered}
$$

To complete the proof, we have only to show the real-diagonalizability of

$$
\xi\left[\begin{array}{lll}
1 & 0 & 0 \\
0 & 0 & 0 \\
0 & 0 & 0
\end{array}\right]+\eta\left[\begin{array}{lll}
0 & 1 & 0 \\
1 & 0 & 1 \\
0 & 0 & 0
\end{array}\right] \quad(\xi, \eta \in \mathbf{R})
$$

is not uniform. For this purpose, it suffices to calculate its three eigenvectors and construct a diagonalizer. See Kasahara-Yamaguti [4] for the detail.

The results obtained in this section are summerized as follows.

Theorem 4.7. Let $\left\langle A_{1}, A_{2}\right\rangle$ be a nondegenerate $3 \times 3$ matrix family. Then the following holds.

1) Suppose $\left\langle A_{1}, A_{2}\right\rangle$ has multiple eigenvalues and is uniformly real-diagonalizable. Then $\left\langle A_{1}, A_{2}\right\rangle$ is equivalent to a hermitian family.

2) The family $\left\langle A_{1}, A_{2}\right\rangle$ is non-uniformly real-diagonalizable (consequently, $\left\langle A_{1}, A_{2}\right\rangle$ must have multiple eigenvalues) if and only if $\left\langle A_{1}, A_{2}\right\rangle$ is equivalent to either

$$
\left\langle\left[\begin{array}{lll}
1 & 0 & 0 \\
0 & 0 & 0 \\
0 & 0 & 0
\end{array}\right],\left[\begin{array}{lll}
0 & 1 & 0 \\
1 & 0 & 1 \\
0 & 0 & 0
\end{array}\right]\right\rangle
$$

or its transpose,

$$
\left\langle\left[\begin{array}{lll}
1 & 0 & 0 \\
0 & 0 & 0 \\
0 & 0 & 0
\end{array}\right],\left[\begin{array}{lll}
0 & 1 & 0 \\
1 & 0 & 0 \\
0 & 1 & 0
\end{array}\right]\right\rangle .
$$




\section{§5. Families Spanmed by Three Matrices}

In this section, we study nondegenerate real-diagonalizable families, say, $\langle A, B, C\rangle$, spanned by three matrices. From the arguments of Section 4 , we may assume that $\langle A, B, C\rangle$ is one of the following three types.

$$
\begin{aligned}
& \left\langle\left[\begin{array}{lll}
1 & 0 & 0 \\
0 & 0 & 0 \\
0 & 0 & 0
\end{array}\right],\left[\begin{array}{lll}
0 & 1 & 0 \\
1 & 0 & 0 \\
0 & 0 & 0
\end{array}\right], C\right\rangle . \\
& \left\langle\left[\begin{array}{lll}
1 & 0 & 0 \\
0 & 0 & 0 \\
0 & 0 & 0
\end{array}\right],\left[\begin{array}{ccc}
0 & \beta_{1} & \beta_{2} \\
\beta_{1} & 1 & 0 \\
\beta_{2} & 0 & -1
\end{array}\right], C\right\rangle \text { with } \beta_{1}, \beta_{2} \text { reals } . \\
& \left\langle\left[\begin{array}{lll}
1 & 0 & 0 \\
0 & 0 & 0 \\
0 & 0 & 0
\end{array}\right],\left[\begin{array}{lll}
0 & 1 & 0 \\
1 & 0 & 1 \\
0 & 0 & 0
\end{array}\right], C\right\rangle .
\end{aligned}
$$

We begin with the first two cases, i.e., the families each of whose members has a right-lower $2 \times 2$ submatrix similar to a real diagonal one. Changing the basis if necessary, such a matrix family must be equivalent to one of the following types.

$$
\begin{aligned}
& \left\langle\left[\begin{array}{lll}
1 & 0 & 0 \\
0 & 0 & 0 \\
0 & 0 & 0
\end{array}\right],\left[\begin{array}{lll}
0 & 1 & 0 \\
1 & 0 & 0 \\
0 & 0 & 0
\end{array}\right],\left[\begin{array}{ccc}
0 & c_{1} & c_{2} \\
c_{3} & 0 & 0 \\
c_{4} & 0 & 0
\end{array}\right]\right\rangle, \\
& \left\langle\left[\begin{array}{lll}
1 & 0 & 0 \\
0 & 0 & 0 \\
0 & 0 & 0
\end{array}\right],\left[\begin{array}{ccc}
0 & \beta_{1} & \beta_{2} \\
\beta_{1} & 1 & 0 \\
\beta_{2} & 0 & -1
\end{array}\right],\left[\begin{array}{ccc}
0 & c_{1} & c_{2} \\
c_{3} & 0 & 0 \\
c_{4} & 0 & 0
\end{array}\right]\right\rangle, \\
& \left\langle\left[\begin{array}{rrr}
1 & 0 & 0 \\
0 & 0 & 0 \\
0 & 0 & 0
\end{array}\right],\left[\begin{array}{rrr}
0 & \alpha & \beta \\
\alpha & 1 & 0 \\
\beta & 0 & -1
\end{array}\right],\left[\begin{array}{lll}
0 & a & b \\
c & 0 & e \\
d & f & 0
\end{array}\right]\right\rangle \quad(e f>0) \text {. }
\end{aligned}
$$

The property ef $>0$ of (5.3) is derived as follows. Since every member of (5.3) must have a right-lower $2 \times 2$ matrix similar to a real diagonal one, we have $e=f=0$ or $e f>0$. In the first case, however, (5.3) reduces to (5.2). So we may assume ef $>0$. Furthermore, we can reduce (5.3) by the similarity transformation with

$$
T=\left[\begin{array}{ccc}
1 & 0 & 0 \\
0 & \sqrt{f / e} & 0 \\
0 & 0 & \sqrt{e / f}
\end{array}\right],
$$

to 


$$
\left\langle\left[\begin{array}{lll}
1 & 0 & 0 \\
0 & 0 & 0 \\
0 & 0 & 0
\end{array}\right],\left[\begin{array}{ccc}
0 & b_{1} & b_{2} \\
b_{3} & 1 & 0 \\
b_{4} & 0 & -1
\end{array}\right],\left[\begin{array}{ccc}
0 & c_{1} & c_{2} \\
c_{3} & 0 & 1 \\
c_{4} & 1 & 0
\end{array}\right]\right\rangle .
$$

We shall treat (5.1), (5.2), (5.3') separately.

Proposition 5.1. The nondegenerate matrix family $\langle A, B, C\rangle$ spanned by

$$
A=\left[\begin{array}{lll}
1 & 0 & 0 \\
0 & 0 & 0 \\
0 & 0 & 0
\end{array}\right], \quad B=\left[\begin{array}{lll}
0 & 1 & 0 \\
1 & 0 & 0 \\
0 & 0 & 0
\end{array}\right], \quad C=\left[\begin{array}{ccc}
0 & c_{1} & c_{2} \\
c_{3} & 0 & 0 \\
c_{4} & 0 & 0
\end{array}\right]
$$

is real-diagonalizable if and only if $\langle A, B, C\rangle$ is equivalent either to

$$
\left\langle\left[\begin{array}{lll}
1 & 0 & 0 \\
0 & 0 & 0 \\
0 & 0 & 0
\end{array}\right],\left[\begin{array}{lll}
0 & 1 & 0 \\
1 & 0 & 0 \\
0 & 0 & 0
\end{array}\right],\left[\begin{array}{ccc}
0 & -c+\frac{1}{c} & c+\frac{1}{c} \\
c-\frac{1}{c} & 0 & 0 \\
c+\frac{1}{c} & 0 & 0
\end{array}\right]\right\rangle
$$

where $c \neq 0$ is an arbitrary complex constant, or to

$$
\left\langle\left[\begin{array}{lll}
1 & 0 & 0 \\
0 & 0 & 0 \\
0 & 0 & 0
\end{array}\right],\left[\begin{array}{lll}
0 & 1 & 0 \\
1 & 0 & 0 \\
0 & 0 & 0
\end{array}\right],\left[\begin{array}{rrr}
0 & -i & -i \\
i & 0 & 0 \\
0 & 0 & 0
\end{array}\right]\right\rangle
$$

or to the transpose of the last;

$$
\left\langle\left[\begin{array}{lll}
1 & 0 & 0 \\
0 & 0 & 0 \\
0 & 0 & 0
\end{array}\right],\left[\begin{array}{lll}
0 & 1 & 0 \\
1 & 0 & 0 \\
0 & 0 & 0
\end{array}\right],\left[\begin{array}{rrr}
0 & -i & 0 \\
i & 0 & 0 \\
i & 0 & 0
\end{array}\right]\right\rangle .
$$

In every of these cases. $\langle A, B, C\rangle$ is uniformly real-diagonalizable.

Proof. Let us first prove the necessity. Clearly, the subfamily

$$
\langle A, \eta B+C\rangle=\left\langle\left[\begin{array}{lll}
1 & 0 & 0 \\
0 & 0 & 0 \\
0 & 0 & 0
\end{array}\right],\left[\begin{array}{ccc}
0 & \eta+c_{1} & c_{2} \\
\eta+c_{3} & 0 & 0 \\
c_{4} & 0 & 0
\end{array}\right]\right\rangle
$$

is real-diagonalizable for an arbitrarily fixed $\eta$. By applying Proposition 4.1, we have

$$
\left(\eta+c_{1}\right)\left(\eta+c_{3}\right)+c_{2} c_{4} \equiv \eta^{2}+\left(c_{1}+c_{3}\right) \eta+c_{1} c_{3}+c_{2} c_{4}>0 .
$$

Because this inequality holds for an arbitrary $\eta \in \mathbb{R}$,

$$
c_{1}+c_{3}
$$


is real. So, replacing $C$ by

$$
C-\frac{1}{2}\left(c_{1}+c_{3}\right) B
$$

we may further assume

$$
c_{3}=-c_{1} \text {. }
$$

From (5.4) and (5.5), we have

$$
-c_{1}^{2}+c_{2} c_{4}>0 \text {. }
$$

Let us first assume

$$
c_{2} c_{4} \neq 0 \text {. }
$$

Then, replacing $C$ by its appropriate real scalar multiple, we may also assume

$$
-c_{1}^{2}+c_{2} c_{4}=4 \text {. }
$$

Now considering a suitable similarity transformation with

$$
\left[\begin{array}{lll}
1 & 0 & 0 \\
0 & 1 & 0 \\
0 & 0 & d
\end{array}\right] \quad(d \neq 0 \text { : complex })
$$

we may further assume

$$
c_{2}=c_{4}
$$

and we have, from (5.7),

$$
-c_{1}^{2}+c_{2}^{2}=4
$$

Now putting

$$
c_{1}=-\left(c-\frac{1}{c}\right)
$$

with some complex $c \neq 0$, we can solve (5.9) with respect to $c_{2}$ and get

$$
c_{2}= \pm\left(c+\frac{1}{c}\right) \text {. }
$$

From (5.5), (5.8), (5.10), (5.11), we have

$$
\langle A, B, C\rangle \sim\left\langle\left[\begin{array}{lll}
1 & 0 & 0 \\
0 & 0 & 0 \\
0 & 0 & 0
\end{array}\right],\left[\begin{array}{lll}
0 & 1 & 0 \\
1 & 0 & 0 \\
0 & 0 & 0
\end{array}\right],\left[\begin{array}{ccc}
0 & -c+\frac{1}{c} & c+\frac{1}{c} \\
c-\frac{1}{c} & 0 & 0 \\
c+\frac{1}{c} & 0 & 0
\end{array}\right]\right\rangle
$$


or

$$
\langle A, B, C\rangle \sim\left\langle\left[\begin{array}{lll}
1 & 0 & 0 \\
0 & 0 & 0 \\
0 & 0 & 0
\end{array}\right],\left[\begin{array}{lll}
0 & 1 & 0 \\
1 & 0 & 0 \\
0 & 0 & 0
\end{array}\right],\left[\begin{array}{ccc}
0 & -c+\frac{1}{c} & -c-\frac{1}{c} \\
c-\frac{1}{c} & 0 & 0 \\
-c-\frac{1}{c} & 0 & 0
\end{array}\right]\right\rangle
$$

However, the second case reduces to the first if we use the similarity transformation with

$$
\left[\begin{array}{rrr}
1 & 0 & 0 \\
0 & 1 & 0 \\
0 & 0 & -1
\end{array}\right]
$$

Thus we have the first family of the requirement.

Let us now consider the case

$$
c_{2} c_{4}=0 \text {. }
$$

From (5.6), we know that $c_{1}$ is purely imaginary. So replacing $C$ by its appropriate real multiple, we have

$$
c_{1}=-i
$$

We may further assume $c_{2} \neq 0$ or $c_{4} \neq 0$ because if $c_{2}=c_{4}=0$ the matrix family is equivalent to (5.12) with $c=i$. Now, using an appropriate similarity transformation with

$$
T=\left[\begin{array}{lll}
1 & 0 & 0 \\
0 & 1 & 0 \\
0 & 0 & d
\end{array}\right] \quad(d \neq 0: \text { complex })
$$

$\langle A, B, C\rangle$ can be reduced to one of the last two families of the requirement.

Let us now prove the uniform real-diagonalizability of the matrix families just obtained. It suffices to consider

$$
\left\langle\left[\begin{array}{lll}
1 & 0 & 0 \\
0 & 0 & 0 \\
0 & 0 & 0
\end{array}\right],\left[\begin{array}{lll}
0 & 1 & 0 \\
1 & 0 & 0 \\
0 & 0 & 0
\end{array}\right],\left[\begin{array}{ccc}
0 & c_{1} & c_{2} \\
-c_{1} & 0 & 0 \\
c_{4} & 0 & 0
\end{array}\right]\right\rangle
$$

where

$$
-c_{1}{ }^{2}+c_{2} c_{4}>0 \text {. }
$$

Put 


$$
\begin{aligned}
\varphi & =\varphi(\eta, \zeta)=\left[\eta^{2}+\left(-c_{1}{ }^{2}+c_{2} c_{4}\right) \zeta^{2}\right]^{1 / 2} \\
S(\eta, \zeta) & =\left[\begin{array}{ccc}
1 & 0 & 0 \\
0 & \left(\eta-c_{1} \zeta\right) / \varphi & -c_{2} \zeta / \varphi \\
0 & c_{4} \zeta / \varphi & \left(\eta+c_{1} \zeta\right) / \varphi
\end{array}\right] \text { for }(\eta, \zeta) \neq(0,0) \\
S(0,0) & =I=\left[\begin{array}{lll}
1 & 0 & 0 \\
0 & 1 & 0 \\
0 & 0 & 1
\end{array}\right] .
\end{aligned}
$$

Thus we obtain the uniformity of $S(\eta, \zeta)$ and $S(\eta, \zeta)^{-1}$ as well as

$$
S(\eta, \zeta)^{-1}\{\xi A+\eta \mathbb{B}+\zeta C\} S(\eta, \zeta)=\left[\begin{array}{ccc}
\xi & \varphi(\eta, \zeta) & 0 \\
\varphi(\eta, \zeta) & 0 & 0 \\
0 & 0 & 0
\end{array}\right]
$$

Thus $\xi A+\eta B+\zeta C$ is uniformly real-symmetrized. Therefore $\langle A, B, C\rangle$ is uniformly real-diagonalizable.

Lemma 5.2. The following matrix families are not equivalent to any hermitian family:

$$
\text { 1) }\left\langle\left[\begin{array}{lll}
1 & 0 & 0 \\
0 & 0 & 0 \\
0 & 0 & 0
\end{array}\right],\left[\begin{array}{lll}
0 & 1 & 0 \\
1 & 0 & 0 \\
0 & 0 & 0
\end{array}\right],\left[\begin{array}{ccc}
0 & -c+\frac{1}{c} & c+\frac{1}{c} \\
c-\frac{1}{c} & 0 & 0 \\
c+\frac{1}{c} & 0 & 0
\end{array}\right]\right\rangle
$$

where the constant $c \neq 0$ satisfies $|c| \neq 1$;

$$
\begin{aligned}
& \text { 2) }\left\langle\left[\begin{array}{lll}
1 & 0 & 0 \\
0 & 0 & 0 \\
0 & 0 & 0
\end{array}\right],\left[\begin{array}{lll}
0 & 1 & 0 \\
1 & 0 & 0 \\
0 & 0 & 0
\end{array}\right],\left[\begin{array}{rrr}
0 & -i & -i \\
i & 0 & 0 \\
0 & 0 & 0
\end{array}\right]\right\rangle \text {; } \\
& \left.2^{\prime}\right\rangle\left\langle\left[\begin{array}{lll}
1 & 0 & 0 \\
0 & 0 & 0 \\
0 & 0 & 0
\end{array}\right],\left[\begin{array}{lll}
0 & 1 & 0 \\
1 & 0 & 0 \\
0 & 0 & 0
\end{array}\right],\left[\begin{array}{rrr}
0 & -i & 0 \\
i & 0 & 0 \\
i & 0 & 0
\end{array}\right]\right\rangle .
\end{aligned}
$$

Proof. In order to prove the lemma by contradiction, we assume that there exists $T$ such that

$$
\mathbb{T}^{-1} A T, \quad T^{-1} B T, \quad T^{-1} C T
$$

are simultaneously hermitian. So we can diagonalize $\mathbb{T}^{-1} A \mathbb{T}$ by a unitary $U$ as following. 


$$
U^{-1} T^{-1} A T U=\left[\begin{array}{lll}
1 & 0 & 0 \\
0 & 0 & 0 \\
0 & 0 & 0
\end{array}\right]=A .
$$

This means that $A$ and $T U$ commute. Hence replacing $T U$ by its appropriate complex scalar multiple if necessary, it has the following form:

$$
T U=\left[\begin{array}{lll}
1 & 0 & 0 \\
0 & a & b \\
0 & c & d
\end{array}\right] \quad(a d-b c \neq 0) .
$$

we define another unitary matrix $U_{1}$ by

$$
U_{1}=\left[\begin{array}{ccc}
1 & 0 & 0 \\
0 & \frac{\bar{a}}{\sqrt{|a|^{2}+|b|^{2}}} & \frac{-b}{\sqrt{|a|^{2}+|b|^{2}}} \\
0 & \frac{\bar{b}}{\sqrt{|a|^{2}+|b|^{2}}} & \frac{a}{\sqrt{|a|^{2}+|b|^{2}}}
\end{array}\right] .
$$

Then $T_{1}=T U U_{1}$ has the following form:

$$
T_{1}=\left[\begin{array}{ccc}
1 & 0 & 0 \\
0 & \alpha & 0 \\
0 & c^{\prime} & d^{\prime}
\end{array}\right]
$$

where $\alpha>0$ is a real, $c^{\prime}$ and $d^{\prime}$ are complex constants. Since

$$
T_{1}^{-1} B T_{1}=\left(U U_{1}\right)^{-1} T^{-1} B T\left(U U_{1}\right)
$$

is hermitian, its $(2,1)$ - and $(1,2)$ - entries are complex conjugate and so are its $(3,1)$ - and $(1,3)$ - entries, namely,

$$
\begin{aligned}
& 1 / \alpha=\alpha>0, \\
& -c^{\prime} / \alpha d^{\prime}=0 .
\end{aligned}
$$

From this we have $\alpha=1$ and $c^{\prime}=0$, that is, $T_{1}$ has the following form;

$$
T_{1}=\left[\begin{array}{lll}
1 & 0 & 0 \\
0 & 1 & 0 \\
0 & 0 & d^{\prime}
\end{array}\right] \quad\left(d^{\prime} \neq 0: \text { complex }\right)
$$

On the other hand,

$$
T_{1}^{-1} C T_{1}=\left(U U_{1}\right)^{-1} T^{-1} C T\left(U U_{1}\right)
$$

must also be hermitian. However, its $(2,1)$ - and $(1,2)$ - entries (or its $(3,1)$ - and $(1,3)$ - entries) are not complex conjugate in each of the cases 1), 2), 2'). This 
fact can be verified by a straightforward calculation. We are thus led to a contradiction.

Now we investigate the families of the form:

$$
\left\langle\left[\begin{array}{lll}
1 & 0 & 0 \\
0 & 0 & 0 \\
0 & 0 & 0
\end{array}\right],\left[\begin{array}{rrr}
1 & * & * \\
* & 1 & 0 \\
* & 0 & -1
\end{array}\right],\left[\begin{array}{lll}
0 & * & * \\
* & 0 & 0 \\
* & 0 & 0
\end{array}\right]\right\rangle
$$

where each $*$ stands for a certain complex constant. Let us prove that they are equivalent to a hermitian family, in a generalized form.

Proposition 5.3. Let a nondegenerate matrix family $\left\langle A, B, C_{1}, \ldots, C_{n}\right\rangle(n \geq 1)$ be spanned by

$$
\begin{aligned}
A & =\left[\begin{array}{lll}
1 & 0 & 0 \\
0 & 0 & 0 \\
0 & 0 & 0
\end{array}\right], \\
B & =\left[\begin{array}{ccc}
0 & \beta_{1}+i \tilde{\beta}_{1} & \beta_{2}+i \tilde{\beta}_{2} \\
\beta_{3}+i \tilde{\beta}_{3} & 1 & 0 \\
\beta_{4}+i \tilde{\beta}_{4} & 0 & -1
\end{array}\right], \\
C_{j} & =\left[\begin{array}{ccc}
0 & \gamma_{j 1}+i \tilde{\gamma}_{j 1} & \gamma_{j 2}+i \tilde{\gamma}_{j 2} \\
\gamma_{j 3}+i \tilde{\gamma}_{j 3} & 0 & 0 \\
\gamma_{j 4}+i \tilde{\gamma}_{j 4} & 0 & 0
\end{array}\right] \quad(j=1, \ldots, n),
\end{aligned}
$$

where $\beta_{k}, \tilde{\beta}_{k}, \gamma_{j k}, \tilde{\gamma}_{j k}(j=1, \ldots, n ; k=1, \ldots, 4)$ are arbitrary real constants. The matrix family $\left\langle A, B, C_{1}, \ldots, C_{n}\right\rangle$ is real-diagonalizable if and only if it is equivalent to a hermitian family.

Proof. It suffices to prove the only-if part. For any fixed $\eta=\left(\eta_{1}, \ldots, \eta_{n}\right) \in$ $\mathbb{R}^{n}$, we have

$$
\begin{aligned}
\langle A, B & \left.+\sum_{j=1}^{n} \eta_{j} C_{j}\right\rangle \\
& =\left\langle\left[\begin{array}{lll}
1 & 0 & 0 \\
0 & 0 & 0 \\
0 & 0 & 0
\end{array}\right],\left[\begin{array}{ccc}
0 & \sigma_{1}(\eta)+i \tilde{\sigma}_{1}(\eta) & \sigma_{2}(\eta)+i \tilde{\sigma}_{2}(\eta) \\
\sigma_{3}(\eta)+i \tilde{\sigma}_{3}(\eta) & 1 & 0 \\
\sigma_{4}(\eta)+i \tilde{\sigma}_{4}(\eta) & 0 & -1
\end{array}\right]\right\rangle
\end{aligned}
$$

is real-diagonalizable where

$$
\begin{array}{ll}
\sigma_{k}(\eta) \equiv\left(\sum_{j=1}^{n} \gamma_{j k} \eta_{j}\right)+\beta_{k} & (k=1, \ldots, 4), \\
\tilde{\sigma}_{k}(\eta) \equiv\left(\sum_{j=1}^{n} \tilde{\gamma}_{j k} \eta_{j}\right)+\tilde{\beta}_{k} & (k=1, \ldots, 4) .
\end{array}
$$

So, from Proposition 4.3, 


$$
\left\{\sigma_{1}(\eta)+i \tilde{\sigma}_{1}(\eta)\right\}\left\{\sigma_{3}(\eta)+i \tilde{\sigma}_{3}(\eta)\right\}>0
$$

or

$$
\sigma_{1}(\eta)+i \tilde{\sigma}_{1}(\eta)=\sigma_{3}(\eta)+i \tilde{\sigma}_{3}(\eta)=0
$$

holds for any arbitrarily fixed $\eta \in \mathbb{R}^{n}$. Similarly,

$$
\left\{\sigma_{2}(\eta)+i \tilde{\sigma}_{2}(\eta)\right\}\left\{\sigma_{4}(\eta)+i \tilde{\sigma}_{4}(\eta)\right\}>0
$$

or

$$
\sigma_{2}(\eta)+i \tilde{\sigma}_{2}(\eta)=\sigma_{4}(\eta)+i \tilde{\sigma}_{4}(\eta)=0
$$

holds for any $\eta \in \mathbb{R}^{n}$. Therefore Lemma 3.2 is applicable. Let us begin with $\sigma_{1}(\eta)+i \tilde{\sigma}_{1}(\eta)$ and $\sigma_{3}(\eta)+i \tilde{\sigma}_{3}(\eta)$. From the last assertion of Lemma 3.2, we have

$$
\operatorname{sgn} \sigma_{1}(\eta) \equiv \operatorname{sgn} \sigma_{3}(\eta), \quad \operatorname{sgn} \tilde{\sigma}_{1}(\eta) \equiv-\operatorname{sgn} \tilde{\sigma}_{3}(\eta) .
$$

Since the polynomials are all linear, these equalities mean that

$$
\sigma_{3}(\eta) \equiv \alpha_{1} \sigma_{1}(\eta), \quad \tilde{\sigma}_{3}(\eta) \equiv-\tilde{\alpha}_{1} \tilde{\sigma}_{1}(\eta)
$$

where $\alpha_{1}>0$ and $\tilde{\alpha}_{1}>0$ are positive constants. Let us show $\tilde{\alpha}_{1}=\alpha_{1}$. We may assume that neither $\sigma_{1}(\eta)$ nor $\tilde{\sigma}_{1}(\eta)$ is identically zero, because otherwise we can clearly equate $\alpha_{1}>0$ and $\tilde{\alpha}_{1}>0$. Recall now that

$$
\begin{aligned}
\left\{\sigma_{1}(\eta)\right. & \left.+i \tilde{\sigma}_{1}(\eta)\right\}\left\{\sigma_{3}(\eta)+i \tilde{\sigma}_{3}(\eta)\right\} \\
& \equiv\left\{\sigma_{1}(\eta)+i \tilde{\sigma}_{1}(\eta)\right\}\left\{\alpha_{1} \sigma_{1}(\eta)-i \tilde{\alpha}_{1} \tilde{\sigma}_{3}(\eta)\right\} \\
& \equiv \alpha_{1}\left\{\sigma_{1}(\eta)\right\}^{2}+\tilde{\alpha}_{1}\left\{\tilde{\sigma}_{1}(\eta)\right\}^{2}+i\left(\alpha_{1}-\tilde{\alpha}_{1}\right) \sigma_{1}(\eta) \tilde{\sigma}_{1}(\eta)
\end{aligned}
$$

must be real for all $\eta \in \mathbb{R}^{n}$. Therefore we must have $\tilde{\alpha}_{1}=\alpha_{1}$. This means

$$
\sigma_{3}(\eta) \equiv \alpha_{1} \sigma_{1}(\eta), \quad \tilde{\sigma}_{3}(\eta) \equiv-\alpha_{1} \tilde{\sigma}_{1}(\eta) \quad\left(\alpha_{1}>0\right)
$$

We can proceed in the same way for the pair $\sigma_{2}(\eta)+i \tilde{\sigma}_{2}(\eta)$ and $\sigma_{4}(\eta)+i \tilde{\sigma}_{4}(\eta)$, and we find a positive constant $\alpha_{2}>0$ such that

$$
\sigma_{4}(\eta) \equiv \alpha_{2} \sigma_{2}(\eta), \quad \tilde{\sigma}_{4}(\eta) \equiv-\alpha_{2} \tilde{\sigma}_{2}(\eta)
$$

From (5.13) and (5.14), we obtain

$$
\begin{array}{ll}
\beta_{3}=\alpha_{1} \beta_{1}, & \tilde{\beta}_{3}=-\alpha_{1} \tilde{\beta}_{1}, \\
\beta_{4}=\alpha_{2} \beta_{2}, & \tilde{\beta}_{4}=-\alpha_{2} \tilde{\beta}_{2}, \\
\gamma_{j 3}=\alpha_{1} \gamma_{j 1}, & \tilde{\gamma}_{j 3}=-\alpha_{1} \tilde{\gamma}_{j 1}, \\
\gamma_{j 4}=\alpha_{2} \gamma_{j 2}, & \tilde{\gamma}_{j 4}=-\alpha_{2} \tilde{\gamma}_{j 2}
\end{array}
$$


with

$$
\alpha_{1}>0, \quad \alpha_{2}>0
$$

Therefore $\left\langle A, B, C_{1}, \ldots, C_{n}\right\rangle$ is equivalent to a certain hermitian family through the similarity transformation with

$$
T=\left[\begin{array}{ccc}
1 & 0 & 0 \\
0 & \sqrt{\alpha_{1}} & 0 \\
0 & 0 & \sqrt{\alpha_{2}}
\end{array}\right]
$$

Thus the proof is completed.

Let us now investigate the families of the form:

$$
\left\langle\left[\begin{array}{lll}
1 & 0 & 0 \\
0 & 0 & 0 \\
0 & 0 & 0
\end{array}\right],\left[\begin{array}{rrr}
0 & * & * \\
* & 1 & 0 \\
* & 0 & -1
\end{array}\right],\left[\begin{array}{ccc}
0 & * & * \\
* & 0 & 1 \\
* & 1 & 0
\end{array}\right]\right\rangle
$$

where each $*$ stands for a certain complex constant.

Proposition 5.4. Let a nondegenerate matrix family $\left\langle A, B_{1}, B_{2}\right\rangle$ be spanned by

$$
\begin{aligned}
A & =\left[\begin{array}{lll}
1 & 0 & 0 \\
0 & 0 & 0 \\
0 & 0 & 0
\end{array}\right], \\
B_{1} & =\left[\begin{array}{ccc}
0 & \beta_{11}+i \tilde{\beta}_{11} & \beta_{12}+i \tilde{\beta}_{12} \\
\beta_{13}+i \tilde{\beta}_{13} & 1 & 0 \\
\beta_{14}+i \tilde{\beta}_{14} & 0 & -1
\end{array}\right], \\
B_{2} & =\left[\begin{array}{ccc}
0 & \beta_{21}+i \tilde{\beta}_{21} & \beta_{22}+i \tilde{\beta}_{22} \\
\beta_{23}+i \tilde{\beta}_{23} & 0 & 1 \\
\beta_{24}+i \tilde{\beta}_{24} & 1 & 0
\end{array}\right],
\end{aligned}
$$

where $\beta_{j k}, \quad \tilde{\beta}_{j k}(j=1,2, k=1,2,3,4)$ are arbitrary real constants. Then $\left\langle A, B_{1}, B_{2}\right\rangle$ is real-diagonalizable if and only if it is either equivalent to

$$
\left\langle\left[\begin{array}{lll}
1 & 0 & 0 \\
0 & 0 & 0 \\
0 & 0 & 0
\end{array}\right],\left[\begin{array}{ccc}
0 & \alpha & -i(\alpha-2 \gamma) \delta \\
\alpha & 1 & 0 \\
i\left(\alpha-2 \gamma^{\prime}\right) \delta & 0 & -1
\end{array}\right],\left[\begin{array}{ccc}
0 & \beta-i \gamma \delta & \gamma-i \beta \delta \\
\beta^{\prime}+i \gamma^{\prime} \delta & 0 & 1 \\
\gamma^{\prime}+i \beta^{\prime} \delta & 1 & 0
\end{array}\right]\right\rangle
$$

where the real constants $\alpha, \beta, \beta^{\prime}, \gamma, \gamma^{\prime}$ and $\delta$ satisfy

$$
\alpha>0, \quad \gamma>\frac{1}{2}\left(\alpha+\frac{\beta^{2}}{\alpha}\right), \quad \gamma^{\prime}>\frac{1}{2}\left(\alpha+\frac{\beta^{2}}{\alpha}\right)
$$


or equivalent to a hermitian family. And in both of these cases, $\left\langle A, B_{1}, B_{2}\right\rangle$ is uniformly real-diagonalizable.

The proof of Proposition 5.4 is somewhat lengthy. So we split it into several lemmas.

Lemma 5.5. Let $A, B_{1}, B_{2}$ be the same as in Proposition 5.4. Let also the polynomial with real coefficients, $\sigma_{1}(\eta), \tilde{\sigma}_{1}(\eta), \sigma_{3}(\eta), \tilde{\sigma}_{3}(\eta)$ be defined as

$$
\begin{aligned}
& \sigma_{1}(\eta) \equiv\left(\eta_{1}{ }^{2}-\eta_{2}{ }^{2}\right)\left(\beta_{11} \eta_{1}+\beta_{12} \eta_{2}\right)+2 \eta_{1} \eta_{2}\left(\beta_{21} \eta_{1}+\beta_{22} \eta_{2}\right), \\
& \tilde{\sigma}_{1}(\eta) \equiv\left(\eta_{1}{ }^{2}-\eta_{2}{ }^{2}\right)\left(\tilde{\beta}_{11} \eta_{1}+\tilde{\beta}_{12} \eta_{2}\right)+2 \eta_{1} \eta_{2}\left(\tilde{\beta}_{21} \eta_{1}+\tilde{\beta}_{22} \eta_{2}\right), \\
& \sigma_{3}(\eta) \equiv\left(\eta_{1}{ }^{2}-\eta_{2}{ }^{2}\right)\left(\beta_{13} \eta_{1}+\beta_{14} \eta_{2}\right)+2 \eta_{1} \eta_{2}\left(\beta_{23} \eta_{1}+\beta_{24} \eta_{2}\right), \\
& \tilde{\sigma}_{3}(\eta) \equiv\left(\eta_{1}{ }^{2}-\eta_{2}{ }^{2}\right)\left(\tilde{\beta}_{13} \eta_{1}+\tilde{\beta}_{14} \eta_{2}\right)+2 \eta_{1} \eta_{2}\left(\tilde{\beta}_{23} \eta_{1}+\tilde{\beta}_{24} \eta_{2}\right) .
\end{aligned}
$$

Suppose further that $\left\langle A, B_{1}, B_{2}\right\rangle$ is real-diagonalizable and is not equivalent to any hermitian family. Then $\sigma_{1}(\eta), \tilde{\sigma}_{1}(\eta), \sigma_{3}(\eta), \tilde{\sigma}_{3}(\eta)$ are factorized as follows:

$$
\begin{array}{ll}
\sigma_{1}(\eta) \equiv \mu(\eta) \varphi(\eta) ; & \tilde{\sigma}_{1}(\eta) \equiv \tilde{\mu}(\eta) \varphi(\eta) ; \\
\sigma_{3}(\eta) \equiv \mu(\eta) \psi(\eta) ; & \tilde{\sigma}_{3}(\eta) \equiv-\tilde{\mu}(\eta) \psi(\eta) .
\end{array}
$$

Here $\varphi(\eta)$ and $\psi(\eta)$ are positive definite quadratic forms without common factors. Further, $\mu(\eta)$ and $\tilde{\mu}(\eta)$ are homogeneous linear polynomials at least one of which does not vanish identically.

Proof. Consider

$$
\left(\eta_{1}^{2}-\eta_{2}{ }^{2}\right) B_{1}+2 \eta_{1} \eta_{2} B_{2} \equiv\left[\begin{array}{ccc}
0 & \rho_{1}(\eta)+i \tilde{\rho}_{1}(\eta) & \rho_{2}(\eta)+i \tilde{\rho}_{2}(\eta) \\
\rho_{3}(\eta)+i \tilde{\rho}_{3}(\eta) & \eta_{1}{ }^{2}-\eta_{2}{ }^{2} & 2 \eta_{1} \eta_{2} \\
\rho_{4}(\eta)+i \tilde{\rho}_{4}(\eta) & 2 \eta_{1} \eta_{2} & -\eta_{1}{ }^{2}+\eta_{2}{ }^{2}
\end{array}\right]
$$

with any fixed $\eta=\left(\eta_{1}, \eta_{2}\right) \in \mathbb{R}^{2} \backslash\{(0,0)\}$. Here

$$
\begin{aligned}
& \rho_{j}(\eta) \equiv \beta_{1 j}\left(\eta_{1}{ }^{2}-\eta_{2}{ }^{2}\right)+2 \beta_{2 j} \eta_{1} \eta_{2}, \\
& \tilde{\rho}_{j}(\eta) \equiv \tilde{\beta}_{1 j}\left(\eta_{1}{ }^{2}-\eta_{2}{ }^{2}\right)+2 \tilde{\beta}_{2 j} \eta_{1} \eta_{2}
\end{aligned}
$$

for $j=1,2,3,4$. Note that

$$
\left\langle A,\left(\eta_{1}{ }^{2}-\eta_{2}^{2}\right) B_{1}+2 \eta_{1} \eta_{2} B_{2}\right\rangle \subset\left\langle A, B_{1}, B_{2}\right\rangle
$$

is clearly real-diagonalizable. Define a real-orthogonal matrix $V(\eta)$ by

$$
V(\eta) \equiv\left[\begin{array}{ccc}
1 & 0 & 0 \\
0 & \eta_{1} /\|\eta\| & -\eta_{2} /\|\eta\| \\
0 & \eta_{2} /\|\eta\| & \eta_{1} /\|\eta\|
\end{array}\right]
$$

where 


$$
\|\eta\|=\left(\eta_{1}{ }^{2}+\eta_{2}{ }^{2}\right)^{1 / 2} .
$$

By the similarity transformation with this $V(\eta)$,

$$
\left\langle A,\left(\eta_{1}{ }^{2}-\eta_{2}{ }^{2}\right) B_{1}+2 \eta_{1} \eta_{2} B_{2}\right\rangle
$$

is equivalent to

$$
\left\langle\left[\begin{array}{lll}
1 & 0 & 0 \\
0 & 0 & 0 \\
0 & 0 & 0
\end{array}\right],\|\eta\|^{-1} \cdot\left[\begin{array}{ccc}
0 & \sigma_{1}(\eta)+i \tilde{\sigma}_{1}(\eta) & \sigma_{2}(\eta)+i \tilde{\sigma}_{2}(\eta) \\
\sigma_{3}(\eta)+i \tilde{\sigma}_{3}(\eta) & \|\eta\|^{3} & 0 \\
\sigma_{4}(\eta)+i \tilde{\sigma}_{4}(\eta) & 0 & -\|\eta\|^{3}
\end{array}\right]\right\rangle
$$

where

$$
\begin{aligned}
& \sigma_{1}(\eta) \equiv\left(\eta_{1}^{2}-\eta_{2}^{2}\right)\left(\beta_{11} \eta_{1}+\beta_{12} \eta_{2}\right)+2 \eta_{1} \eta_{2}\left(\beta_{21} \eta_{1}+\beta_{22} \eta_{2}\right) \\
& \tilde{\sigma}_{1}(\eta) \equiv\left(\eta_{1}{ }^{2}-\eta_{2}{ }^{2}\right)\left(\tilde{\beta}_{11} \eta_{1}+\tilde{\beta}_{12} \eta_{2}\right)+2 \eta_{1} \eta_{2}\left(\tilde{\beta}_{21} \eta_{1}+\tilde{\beta}_{22} \eta_{2}\right) \\
& \sigma_{2}(\eta) \equiv\left(\eta_{1}{ }^{2}-\eta_{2}^{2}\right)\left(\beta_{12} \eta_{1}-\beta_{11} \eta_{2}\right)+2 \eta_{1} \eta_{2}\left(\beta_{22} \eta_{1}-\beta_{21} \eta_{2}\right) \\
& \tilde{\sigma}_{2}(\eta) \equiv\left(\eta_{1}{ }^{2}-\eta_{2}{ }^{2}\right)\left(\tilde{\beta}_{12} \eta_{1}-\tilde{\beta}_{11} \eta_{2}\right)+2 \eta_{1} \eta_{2}\left(\tilde{\beta}_{22} \eta_{1}-\tilde{\beta}_{21} \eta_{2}\right) \\
& \sigma_{3}(\eta) \equiv\left(\eta_{1}{ }^{2}-\eta_{2}{ }^{2}\right)\left(\beta_{13} \eta_{1}+\beta_{14} \eta_{2}\right)+2 \eta_{1} \eta_{2}\left(\beta_{23} \eta_{1}+\beta_{24} \eta_{2}\right) \\
& \tilde{\sigma}_{3}(\eta) \equiv\left(\eta_{1}{ }^{2}-\eta_{2}{ }^{2}\right)\left(\tilde{\beta}_{13} \eta_{1}+\tilde{\beta}_{14} \eta_{2}\right)+2 \eta_{1} \eta_{2}\left(\tilde{\beta}_{23} \eta_{1}+\tilde{\beta}_{24} \eta_{2}\right) \\
& \sigma_{4}(\eta) \equiv\left(\eta_{1}{ }^{2}-\eta_{2}{ }^{2}\right)\left(\beta_{14} \eta_{1}-\beta_{13} \eta_{2}\right)+2 \eta_{1} \eta_{2}\left(\beta_{24} \eta_{1}-\beta_{23} \eta_{2}\right) \\
& \tilde{\sigma}_{4}(\eta) \equiv\left(\eta_{1}{ }^{2}-\eta_{2}{ }^{2}\right)\left(\tilde{\beta}_{14} \eta_{1}-\tilde{\beta}_{13} \eta_{2}\right)+2 \eta_{1} \eta_{2}\left(\tilde{\beta}_{24} \eta_{1}-\tilde{\beta}_{23} \eta_{2}\right) .
\end{aligned}
$$

Note that

$$
\begin{array}{ll}
\sigma_{2}\left(\eta_{1}, \eta_{2}\right) \equiv \sigma_{1}\left(\eta_{2},-\eta_{1}\right), & \tilde{\sigma}_{2}\left(\eta_{1}, \eta_{2}\right) \equiv \tilde{\sigma}_{1}\left(\eta_{2},-\eta_{1}\right) \\
\sigma_{4}\left(\eta_{1}, \eta_{2}\right) \equiv \sigma_{3}\left(\eta_{2},-\eta_{1}\right), & \tilde{\sigma}_{4}\left(\eta_{1}, \eta_{2}\right) \equiv \tilde{\sigma}_{3}\left(\eta_{2},-\eta_{1}\right) .
\end{array}
$$

From Proposition 4.3, either

$$
\left\{\sigma_{1}(\eta)+i \tilde{\sigma}_{1}(\eta)\right\}\left\{\sigma_{3}(\eta)+i \tilde{\sigma}_{3}(\eta)\right\}>0
$$

or

$$
\sigma_{1}(\eta)+i \tilde{\sigma}_{1}(\eta)=\sigma_{3}(\eta)+i \tilde{\sigma}_{3}(\eta)=0
$$

holds for any $\eta \in \mathbb{R}^{2}$. So Lemma 3.2 is applicable and we have

$$
\begin{array}{ll}
\sigma_{1}(\eta) \equiv \mu(\eta) \varphi(\eta), & \tilde{\sigma}_{1}(\eta) \equiv \tilde{\mu}(\eta) \varphi(\eta), \\
\sigma_{3}(\eta) \equiv \mu(\eta) \psi(\eta), & \tilde{\sigma}_{3}(\eta) \equiv-\tilde{\mu}(\eta) \psi(\eta)
\end{array}
$$

where $\mu(\eta), \tilde{\mu}(\eta), \varphi(\eta), \psi(\eta)$ are homogeneous polynomials with real coefficients such that

$$
\operatorname{sgn} \varphi(\eta)=\operatorname{sgn} \psi(\eta)
$$


for all $\eta \in \mathbb{R}^{2}$. The last fact is a consequence of the following. The polynomials $\mu(\eta)$ and $\tilde{\mu}(\eta)$ have no common (nontrivial) real zero points because they are homogeneous polynomials with two variables that have no common factors. Note also that $\varphi(\eta)$ and $\psi(\eta)$ are of the same degree equal to or less than three (see (5.15) and (5.16) and observe that $\sigma_{1}(\eta)$ and $\sigma_{3}(\eta)$ are both cubic).

Before proceeding further, let us prove if $\varphi(\eta)$ is a positive constant multiple of $\psi(\eta)$ then $\left\langle A, B_{1}, B_{2}\right\rangle$ is equivalent to a certain hermitian family. Putting

$$
\psi(\eta) \equiv \alpha \varphi(\eta)
$$

with a real constant $\alpha>0$, we obtain

$$
\sigma_{3}\left(\eta_{1}, \eta_{2}\right) \equiv \alpha \sigma_{1}\left(\eta_{1}, \eta_{2}\right)
$$

From the definition of $\sigma_{1}(\eta)$ and $\sigma_{3}(\eta)$, this means

$$
\begin{array}{cc}
\beta_{j 3}=\alpha \beta_{j 1}, & \beta_{j 4}=\alpha \beta_{j 2}, \\
\tilde{\beta}_{j 3}=-\alpha \tilde{\beta}_{j 1}, & \tilde{\beta}_{j 4}=-\alpha \tilde{\beta}_{j 2},
\end{array}
$$

for $j=1,2$. Then through the similarity transformation with

$$
T=\left[\begin{array}{ccc}
\sqrt{\alpha} & 0 & 0 \\
0 & 1 & 0 \\
0 & 0 & 1
\end{array}\right],
$$

$\left\langle A, B_{1}, B_{2}\right\rangle$ is equivalent to a hermitian family.

In order to make our problem easier, let us divide the case according to the degree of $\varphi(\eta)$ and $\psi(\eta)$. Assume first that they are constants or linear polynomials. Then clearly (5.17) means that each of them is a positive constant multiple of the other. Therefore $\left\langle A, B_{1}, B_{2}\right\rangle$ is equivalent to a hermitian family and we may exclude this case. Assume now that they are (homogeneous) quadratic polynomials. If one of them is an indefinite form, the zero points of $\varphi(\eta)$ and $\psi(\eta)$ on the unit circle of $\mathbf{R}^{2}$ would coincide by virtue of (5.17) and so we would have $\varphi(\eta) \equiv \alpha \psi(\eta)$ for some positive constant $\alpha$, and hence $\left\langle A, B_{1}, B_{2}\right\rangle$ would be a hermitian family. Therefore, $\varphi(\eta)$ and $\psi(\eta)$ must be definite forms, which can be assumed to be positive, because otherwise we may take $-\varphi(\eta),-\psi(\eta),-\mu(\eta)$ and $-\tilde{\mu}(\eta)$ instead of $\varphi(\eta), \psi(\eta), \mu(\eta)$ and $\tilde{\mu}(\eta)$. Also, we can exclude the case where $\varphi(\eta) / \psi(\eta)$ is a constant, as above. Thus the lemma is proved when $\varphi$ and $\psi$ are quadratic. Assume finally that $\varphi\left(\eta_{1}, \eta_{2}\right)$ and $\psi\left(\eta_{1}, \eta_{2}\right)$ are (homogeneous) cubic polynomials. In this case, $\varphi$ and $\psi$ have three complex linear factors of which just one or three are real. And their real linear factors must be common by virtue of (5.17). Therefore, by the same argument as in the quadratic case, two cases are possible, namely either $\varphi(\eta)$ and $\psi(\eta)$ have the form: 


$$
\begin{aligned}
& \varphi(\eta) \equiv \theta(\eta) \tilde{\varphi}(\eta) \\
& \psi(\eta) \equiv \theta(\eta) \tilde{\psi}(\eta)
\end{aligned}
$$

where $\theta(\eta)$ is linear and both of $\tilde{\varphi}(\eta)$ and $\tilde{\psi}(\eta)$ are (distinct) positive definite quadratics, or each of them is a positive constant multiple of the other (see (5.17) again). The second case is impossible because it implies that $\left\langle A, B_{1}, B_{2}\right\rangle$ is equivalent to a hermitian family. So, only the first case can occur. Then, regarding $\mu(\eta) \theta(\eta)$ as $\mu(\eta), \tilde{\mu}(\eta) \theta(\eta)$ as $\tilde{\mu}(\eta), \tilde{\varphi}(\eta)$ as $\varphi(\eta), \tilde{\psi}(\eta)$ as $\psi(\eta)$ in (5.15) and (5.16), the proof of the lemma is completed also in the present case.

Lemma 5.6. Let the assumptions be the same as in Lemma 5.5. Then $\left\langle A, B_{1}, B_{2}\right\rangle$ is equivalent to a matrix family of the same form but with additional restrictions as follows:

$$
\tilde{\beta}_{11}=\tilde{\beta}_{13}=0, \quad \beta_{12}=\beta_{14}=0 .
$$

Proof. Let $\sigma_{1}(\eta)$ and $\sigma_{2}(\eta)$ be the same as in the proof of Lemma 5.5. Now we prove that a value of

$$
\begin{aligned}
\sigma_{2}\left(\eta_{1}, \eta_{2}\right)+i \tilde{\sigma}_{2}\left(\eta_{1}, \eta_{2}\right) & \equiv \sigma_{1}\left(\eta_{2},-\eta_{1}\right)+i \tilde{\sigma}_{1}\left(\eta_{2},-\eta_{1}\right) \\
& \equiv \varphi\left(\eta_{2},-\eta_{1}\right)\left\{\mu\left(\eta_{2},-\eta_{1}\right)+i \tilde{\mu}\left(\eta_{2},-\eta_{1}\right)\right\}
\end{aligned}
$$

becomes the product of

$$
\sigma_{1}\left(\eta_{1}, \eta_{2}\right)+i \tilde{\sigma}_{1}\left(\eta_{1}, \eta_{2}\right) \equiv \varphi\left(\eta_{1}, \eta_{2}\right)\left\{\mu\left(\eta_{1}, \eta_{2}\right)+i \tilde{\mu}\left(\eta_{1}, \eta_{2}\right)\right\}
$$

and a purely imaginary number for some $\left(\eta_{1}, \eta_{2}\right) \in \mathbb{R}^{2} \backslash\{(0,0)\}$. Let us express the two linear forms $\mu$ and $\tilde{\mu}$ as

$$
\begin{aligned}
& \mu\left(\eta_{1}, \eta_{2}\right) \equiv \mu_{1} \eta_{1}+\mu_{2} \eta_{2}, \\
& \tilde{\mu}\left(\eta_{1}, \eta_{2}\right) \equiv \tilde{\mu}_{1} \eta_{1}+\tilde{\mu}_{2} \eta_{2}
\end{aligned}
$$

with some real constants $\mu_{1}, \mu_{2}, \tilde{\mu}_{1}, \tilde{\mu}_{2}$. Thus we have only to find $\left(\eta_{1}, \eta_{2}\right) \in \mathbb{R}^{2}$ such that

$$
\mu\left(\eta_{2},-\eta_{1}\right)+i \tilde{\mu}\left(\eta_{2},-\eta_{1}\right) \equiv \mu_{1} \eta_{2}-\mu_{2} \eta_{1}+i\left(\tilde{\mu}_{1} \eta_{2}-\tilde{\mu}_{2} \eta_{1}\right)
$$

and

$$
i\left\{\mu\left(\eta_{1}, \eta_{2}\right)+i \tilde{\mu}\left(\eta_{1}, \eta_{2}\right)\right\} \equiv-\left(\tilde{\mu}_{1} \eta_{1}+\tilde{\mu}_{2} \eta_{2}\right)+i\left(\mu_{1} \eta_{1}+\mu_{2} \eta_{2}\right)
$$

are linearly dependent over the field of real numbers. For this purpose, let us consider the real zero points of

$$
\begin{aligned}
& \left(\mu_{1} \eta_{2}-\mu_{2} \eta_{1}\right)\left(\mu_{1} \eta_{1}+\mu_{2} \eta_{2}\right)+\left(\tilde{\mu}_{1} \eta_{2}-\tilde{\mu}_{2} \eta_{1}\right)\left(\tilde{\mu}_{1} \eta_{1}+\tilde{\mu}_{2} \eta_{2}\right) \\
& \quad \equiv-\left(\mu_{1} \mu_{2}+\tilde{\mu}_{1} \tilde{\mu}_{2}\right) \eta_{1}{ }^{2}+\left(\mu_{1}{ }^{2}-\mu_{2}{ }^{2}+\tilde{\mu}_{1}{ }^{2}-\tilde{\mu}_{2}{ }^{2}\right) \eta_{1} \eta_{2}+\left(\mu_{1} \mu_{2}+\tilde{\mu}_{1} \tilde{\mu}_{2}\right) \eta_{2}{ }^{2} .
\end{aligned}
$$


This polynomial in $\eta_{1}, \eta_{2}$ has nontrivial real zero points because its coefficients of $\eta_{1}{ }^{2}$ and $\eta_{2}{ }^{2}$ are of opposite signs. Fixing now $\left(\eta_{1}, \eta_{2}\right)$ as such a nontrivial zero point, we find that

$$
\sigma_{2}\left(\eta_{1}, \eta_{2}\right)+i \tilde{\sigma}_{2}\left(\eta_{1}, \eta_{2}\right)
$$

is the product of

$$
\sigma_{1}\left(\eta_{1}, \eta_{2}\right)+i \tilde{\sigma}_{1}\left(\eta_{1}, \eta_{2}\right)
$$

and a purely imaginary number for the above fixed $\left(\eta_{1}, \eta_{2}\right) \in \mathbb{R}^{2} \backslash\{(0,0)\}$.

Through the similarity transformation with

$$
V \equiv\left[\begin{array}{ccc}
1 & 0 & 0 \\
0 & \eta_{1} /\|\eta\| & -\eta_{2} /\|\eta\| \\
0 & \eta_{2} /\|\eta\| & \eta_{1} /\|\eta\|
\end{array}\right],
$$

we can reduce the matrix family

$$
\left\langle A,\left(\eta_{1}{ }^{2}-\eta_{2}{ }^{2}\right) B_{1}+2 \eta_{1} \eta_{2} B_{2},-2 \eta_{1} \eta_{2} B_{1}+\left(\eta_{1}{ }^{2}-\eta_{2}{ }^{2}\right) B_{2}\right\rangle
$$

to the one where the ratio of the $(1,2)$ - and the $(1,3)$ - entry of $B_{1}$ is purely imaginary. Hence we can assume the $(1,2)$ - entry of $B_{1}$ is real and its $(1,3)$ entry is purely imaginary by use of the similarity transformation with

$$
T=\left[\begin{array}{lll}
c & 0 & 0 \\
0 & 1 & 0 \\
0 & 0 & 1
\end{array}\right] \quad(c \neq 0: \text { complex })
$$

Finally, we can conclude that $(2,1)$ - entry as well as $(1,2)$ - is real and that $(3,1)$ - entry as well as $(1,3)$ - is purely imaginary. This is obtained by applying Proposition 4.3 to $\left\langle A, B_{1}\right\rangle$. Thus the proof is complete.

We can thus specify $B_{1}$ in $\left\langle A, B_{1}, B_{2}\right\rangle$ by Lemma 5.6. Now let us further specify $B_{1}$ by the following Lemma 5.7.

Lemma 5.7. Let a nondegenerate matrix family $\left\langle A, B_{1}, B_{2}\right\rangle$ be spanned by

$$
\begin{aligned}
A & =\left[\begin{array}{lll}
1 & 0 & 0 \\
0 & 0 & 0 \\
0 & 0 & 0
\end{array}\right], \\
B_{1} & =\left[\begin{array}{ccc}
0 & \beta_{11} & i \tilde{\beta}_{12} \\
\beta_{13} & 1 & 0 \\
i \tilde{\beta}_{14} & 0 & -1
\end{array}\right], \\
B_{2} & =\left[\begin{array}{ccc}
0 & \beta_{21}+i \tilde{\beta}_{21} & \beta_{22}+i \tilde{\beta}_{22} \\
\beta_{23}+i \tilde{\beta}_{23} & 0 & 1 \\
\beta_{24}+i \tilde{\beta}_{24} & 1 & 0
\end{array}\right],
\end{aligned}
$$


where $\beta_{j k}, \tilde{\beta}_{j k}(j=1,2, k=1,2,3,4)$ are arbitrary real constants. Suppose that $\left\langle A, B_{1}, B_{2}\right\rangle$ is real-diagonalizable and is not equivalent to any hermitian family. Then $\left\langle A, B_{1}, B_{2}\right\rangle$ is equivalent to a matrix family of the same form but with an additional condition:

$$
\beta_{11}=\beta_{13}>0 \text {. }
$$

Proof. Note first that either $\beta_{11} \neq 0$ or $\tilde{\beta}_{12} \neq 0$ holds, because otherwise (i.e., $\beta_{11}=\tilde{\beta}_{11}=\beta_{12}=\tilde{\beta}_{12}=0$ ) Lemma 5.5 yields a contradiction to the factorization of $\sigma_{1}(\eta)$ and $\tilde{\sigma}_{1}(\eta)$. Note next that the case $\beta_{11}=0$ and $\tilde{\beta}_{12} \neq 0$, can be reduced to the case $\beta_{11} \neq 0$, by the successive use of the two similarity transformations with

$$
\left[\begin{array}{lll}
0 & 1 & 0 \\
1 & 0 & 0 \\
0 & 0 & 1
\end{array}\right], \quad\left[\begin{array}{lll}
i & 0 & 0 \\
0 & 1 & 0 \\
0 & 0 & 1
\end{array}\right]
$$

respectively. Let us now apply Proposition 4.3 to $\left\langle A, B_{1}\right\rangle$ with $\beta_{11} \neq 0$. Thus we have

$$
\beta_{11} \beta_{13}>0 \text {. }
$$

Hence, by use of the similarity transformation with some matrix

$$
\left[\begin{array}{lll}
\rho & 0 & 0 \\
0 & 1 & 0 \\
0 & 0 & 1
\end{array}\right] \quad(\rho \neq 0: \text { real })
$$

$\left\langle A, B_{1}, B_{2}\right\rangle$ turns out to satisfy

$$
\beta_{11}=\beta_{13}>0 \text {. }
$$

Thus the proof is complete.

Lemma 5.8. Let the assumptions be the same as in Lemma 5.7. Let also

$$
\beta_{11}=\beta_{13}>0
$$

be fulfilled. Then there exists a real $\delta$ such that

$$
\begin{aligned}
& \beta_{22}>\frac{1}{2}\left(\beta_{11}+\beta_{21}{ }^{2} / \beta_{11}\right), \\
& \tilde{\beta}_{12}=-\delta\left(\beta_{11}-2 \beta_{22}\right), \quad \tilde{\beta}_{21}=-\delta \beta_{22}, \quad \tilde{\beta}_{22}=-\delta \beta_{21}, \\
& \beta_{24}>\frac{1}{2}\left(\beta_{13}+\beta_{23}{ }^{2} / \beta_{13}\right), \\
& \tilde{\beta}_{14}=\delta\left(\beta_{13}-2 \beta_{24}\right), \quad \tilde{\beta}_{23}=\delta \beta_{24}, \quad \tilde{\beta}_{24}=\delta \beta_{23} .
\end{aligned}
$$

Proof. In the present situation, $\sigma_{1}(\eta), \tilde{\sigma}_{1}(\eta), \sigma_{3}(\eta)$ and $\tilde{\sigma}_{3}(\eta)$ in Lemma 5.5 take the form 


$$
\begin{aligned}
& \sigma_{1}(\eta) \equiv \eta_{1}\left\{\beta_{11}\left(\eta_{1}{ }^{2}-\eta_{2}{ }^{2}\right)+2 \beta_{21} \eta_{1} \eta_{2}+2 \beta_{22} \eta_{2}{ }^{2}\right\}, \\
& \tilde{\sigma}_{1}(\eta) \equiv \eta_{2}\left\{\tilde{\beta}_{12}\left(\eta_{1}{ }^{2}-\eta_{2}{ }^{2}\right)+2 \tilde{\beta}_{21} \eta_{1}{ }^{2}+2 \tilde{\beta}_{22} \eta_{1} \eta_{2}\right\}, \\
& \sigma_{3}(\eta) \equiv \eta_{1}\left\{\beta_{13}\left(\eta_{1}{ }^{2}-\eta_{2}{ }^{2}\right)+2 \beta_{23} \eta_{1} \eta_{2}+2 \beta_{24} \eta_{2}{ }^{2}\right\}, \\
& \tilde{\sigma}_{3}(\eta) \equiv \eta_{2}\left\{\tilde{\beta}_{14}\left(\eta_{1}{ }^{2}-\eta_{2}{ }^{2}\right)+2 \tilde{\beta}_{23} \eta_{1}{ }^{2}+2 \tilde{\beta}_{24} \eta_{1} \eta_{2}\right\} .
\end{aligned}
$$

And the same lemma assures that $\sigma_{1}(\eta) / \eta_{1}$ and $\sigma_{3}(\eta) / \eta_{1}$ are positive definite quadratic forms (cf. $\beta_{11}=\beta_{13}>0$ ). It also assures that there exists a real $\delta$ (possibly zero) such that

$$
\tilde{\sigma}_{1}(\eta) / \eta_{2} \equiv-\delta \sigma_{1}(\eta) / \eta_{1}, \quad \tilde{\sigma}_{3}(\eta) / \eta_{2} \equiv \delta \sigma_{3}(\eta) / \eta_{1} .
$$

The conclusion follows immediately from these facts.

Let us consider the converse.

Lemma 5.9. Let

$$
\begin{aligned}
& \beta_{11}=\beta_{13}>0, \\
& \beta_{22}>\frac{1}{2}\left(\beta_{11}+\beta_{21}{ }^{2} / \beta_{11}\right), \\
& \tilde{\beta}_{12}=-\delta\left(\beta_{11}-2 \beta_{22}\right), \quad \tilde{\beta}_{21}=-\delta \beta_{22}, \quad \tilde{\beta}_{22}=-\delta \beta_{21}, \\
& \beta_{24}>\frac{1}{2}\left(\beta_{13}+\beta_{23}{ }^{2} / \beta_{13}\right), \\
& \tilde{\beta}_{14}=\delta\left(\beta_{13}-2 \beta_{24}\right), \quad \tilde{\beta}_{23}=\delta \beta_{24}, \quad \tilde{\beta}_{24}=\delta \beta_{23}
\end{aligned}
$$

be fulfilled for some real $\delta$. Then the matrix family $\left\langle A, B_{1}, B_{2}\right\rangle$ spanned by

$$
\begin{aligned}
A & =\left[\begin{array}{lll}
1 & 0 & 0 \\
0 & 0 & 0 \\
0 & 0 & 0
\end{array}\right], \\
B_{1} & =\left[\begin{array}{rrr}
0 & \beta_{11} & i \tilde{\beta}_{12} \\
\beta_{13} & 1 & 0 \\
i \tilde{\beta}_{14} & 0 & -1
\end{array}\right], \\
B_{2} & =\left[\begin{array}{ccc}
0 & \beta_{21}+i \tilde{\beta}_{21} & \beta_{22}+i \bar{\beta}_{22} \\
\beta_{23}+i \tilde{\beta}_{23} & 0 & 1 \\
\beta_{24}+i \tilde{\beta}_{24} & 1 & 0
\end{array}\right]
\end{aligned}
$$

is uniformly real-diagonalizable.

Proof. Let us diagonalize

$$
M\left(\xi, \eta_{1}, \eta_{2}\right) \equiv \xi A+\left(\eta_{1}^{2}-\eta_{2}^{2}\right) B_{1}+2 \eta_{1} \eta_{2} B_{2}
$$

Note that 


$$
\left(\eta_{1}, \eta_{2}\right) \rightarrow\left(\eta_{1}^{2}-\eta_{2}^{2}, 2 \eta_{1} \eta_{2}\right)
$$

is a map from $\mathbb{R}^{2}$ onto $\mathbb{R}^{2}$ because

$$
\left(\eta_{1}+i \eta_{2}\right)^{2}=\left(\eta_{1}^{2}-\eta_{2}^{2}\right)+2 i \eta_{1} \eta_{2} .
$$

Let $V(\eta) \equiv V\left(\eta_{1}, \eta_{2}\right)$ be a real orthogonal matrix defined by

$$
V(\eta) \equiv\left[\begin{array}{ccc}
1 & 0 & 0 \\
0 & \eta_{1} /\|\eta\| & -\eta_{2} /\|\eta\| \\
0 & \eta_{2} /\|\eta\| & \eta_{1} /\|\eta\|
\end{array}\right] \quad(\eta \neq(0,0))
$$

and

$$
V(0,0)=I=\left[\begin{array}{lll}
1 & 0 & 0 \\
0 & 1 & 0 \\
0 & 0 & 1
\end{array}\right]
$$

Thus, for $\eta \neq(0,0)$, we have

$$
\begin{aligned}
& V(\eta)^{-1} M\left(\xi, \eta_{1}, \eta_{2}\right) V(\eta) \\
& \quad=\frac{1}{\|\eta\|}\left[\begin{array}{ccc}
\xi & \left(\eta_{1}-i \delta \eta_{2}\right) \varphi\left(\eta_{1}, \eta_{2}\right) & \left(\eta_{2}+i \delta \eta_{1}\right) \varphi\left(\eta_{2},-\eta_{1}\right) \\
\left(\eta_{1}+i \delta \eta_{2}\right) \psi\left(\eta_{1}, \eta_{2}\right) & \|\eta\|^{3} & 0 \\
\left(\eta_{2}-i \delta \eta_{1}\right) \psi\left(\eta_{2},-\eta_{1}\right) & 0 & -\|\eta\|^{3}
\end{array}\right]
\end{aligned}
$$

Here

$$
\begin{aligned}
& \varphi\left(\eta_{1}, \eta_{2}\right) \equiv \beta_{11}\left(\eta_{1}{ }^{2}-\eta_{2}{ }^{2}\right)+2 \beta_{21} \eta_{1} \eta_{2}+2 \beta_{22} \eta_{2}{ }^{2} \\
& \psi\left(\eta_{1}, \eta_{2}\right) \equiv \beta_{13}\left(\eta_{1}{ }^{2}-\eta_{2}{ }^{2}\right)+2 \beta_{23} \eta_{1} \eta_{2}+2 \beta_{24} \eta_{2}{ }^{2}
\end{aligned}
$$

are positive definite quadratic forms as a consequence of the assumption. (See also the comments in the first part of the proof of Lemma 5.5). Let us now introduce a diagonal matrix $D(\eta)$ by

$$
D\left(\eta_{1}, \eta_{2}\right) \equiv\left[\begin{array}{ccc}
1 & 0 & 0 \\
0 & \left(\frac{\psi\left(\eta_{1}, \eta_{2}\right)}{\varphi\left(\eta_{1}, \eta_{2}\right)}\right)^{1 / 2} & 0 \\
0 & 0 & \left(\frac{\psi\left(\eta_{2},-\eta_{1}\right)}{\varphi\left(\eta_{2},-\eta_{1}\right)}\right)^{1 / 2}
\end{array}\right]
$$

for $\eta \neq(0,0)$ and

$$
D(0,0)=I=\left[\begin{array}{lll}
1 & 0 & 0 \\
0 & 1 & 0 \\
0 & 0 & 1
\end{array}\right]
$$


Thus we know that

$$
(V(\eta) D(\eta))^{-1} M\left(\xi, \eta_{1}, \eta_{2}\right) V(\eta) D(\eta)
$$

is hermitian and that

$$
\|V(\eta) D(\eta)\|, \quad\left\|(V(\eta) D(\eta))^{-1}\right\| \leq \mathrm{const} .
$$

Hence $M\left(\xi, \eta_{1}, \eta_{2}\right)$ is uniformly real-diagonalizable.

Proof of Proposition 5.4. To prove the necessity, we specify $B_{1}$ by successive use of Lemma 5.6 and 5.7. Then Lemma 5.8 proves the necessity. To prove the sufficiency, we have only to apply Lemma 5.9.

The matrix family indicated in Proposition 5.4 is generically not equivalent to any hermitian family as will be proved in the following proposition.

Proposition 5.10. Let the matrix family $\left\langle A, B_{1}, B_{2}\right\rangle$ be spanned by

$$
\begin{aligned}
A & =\left[\begin{array}{lll}
1 & 0 & 0 \\
0 & 0 & 0 \\
0 & 0 & 0
\end{array}\right], \\
B_{1} & =\left[\begin{array}{ccc}
0 & \alpha & -i(\alpha-2 \gamma) \delta \\
\alpha & 1 & 0 \\
i\left(\alpha-2 \gamma^{\prime}\right) \delta & 0 & -1
\end{array}\right], \\
B_{2} & =\left[\begin{array}{ccc}
0 & \beta-i \gamma \delta & \gamma-i \beta \delta \\
\beta^{\prime}+i \gamma^{\prime} \delta & 0 & 1 \\
\gamma^{\prime}+i \beta^{\prime} \delta & 1 & 0
\end{array}\right],
\end{aligned}
$$

where the real constants $\alpha, \beta, \beta^{\prime}, \gamma, \gamma^{\prime}, \delta$ satisfy

$$
\alpha>0, \quad\left|\beta-\beta^{\prime}\right|+\left|\gamma-\gamma^{\prime}\right|>0 .
$$

Then $\left\langle A, B_{1}, B_{2}\right\rangle$ is not equivalent to any hermitian family.

Proof. Assume, to the contrary, that there exists a nonsingular $T$ such that

$$
T^{-1} A T, \quad T^{-1} B_{1} T, \quad T^{-1} B_{2} T
$$

are simultaneously hermitian. Now we can proceed just in the same way as in the proof of Lemma 5.2 and we may assume that

$$
T=\left[\begin{array}{lll}
1 & 0 & 0 \\
0 & \rho & 0 \\
0 & b & d
\end{array}\right] \quad(\rho>0: \text { real, } b, d: \text { complex }) .
$$

Further the $(2,3)$ - and $(3,2)$ - entries of $T^{-1} B_{1} T$ must be complex conjugate, and so must its $(1,2)$ - and $(2,1)$ - entries. From this, we have $\rho=1, b=0$, 
namely,

$$
T=\left[\begin{array}{lll}
1 & 0 & 0 \\
0 & 1 & 0 \\
0 & 0 & d
\end{array}\right] \quad(d: \text { complex })
$$

Let us now consider $T^{-1} B_{2} T$. Its $(2,3)$ - and $(3,2)$ - entries must be complex conjugate, namely, $\bar{d}=d^{-1}$. This means that $|d|=1$ and $T$ is unitary. Therefore $A, B_{1}, B_{2}$ themselves must be simultaneously hermitian. This contradicts the assumptions.

Let us work on the third matrix families mentioned at the beginning of this section, namely,

$$
\left\langle\left[\begin{array}{lll}
1 & 0 & 0 \\
0 & 0 & 0 \\
0 & 0 & 0
\end{array}\right],\left[\begin{array}{lll}
0 & 1 & 0 \\
1 & 0 & 1 \\
0 & 0 & 0
\end{array}\right], C\right\rangle
$$

with some matrix $C$, or its transposed family. Note that they are nonuniformly real-diagonalizable by virtue of Proposition 4.6. We shall consider (5.18) without loss of generality.

Proposition 5.11. Let a nondegenerate matrix family $\langle A, B, C\rangle$ be spanned by

$$
\begin{aligned}
& A=\left[\begin{array}{lll}
1 & 0 & 0 \\
0 & 0 & 0 \\
0 & 0 & 0
\end{array}\right], \quad B=\left[\begin{array}{lll}
0 & 1 & 0 \\
1 & 0 & 1 \\
0 & 0 & 0
\end{array}\right], \\
& C=\left[\begin{array}{ccc}
0 & \gamma_{1}+i \tilde{\gamma}_{1} & \gamma_{2}+i \tilde{\gamma}_{2} \\
\gamma_{3}+i \tilde{\gamma}_{3} & 0 & \gamma_{5}+i \tilde{\gamma}_{5} \\
\gamma_{4}+i \tilde{\gamma}_{4} & 0 & 0
\end{array}\right] \neq 0
\end{aligned}
$$

where $\gamma_{j}(j=1, \ldots, 5), \quad \tilde{\gamma}_{j}(j=1, \ldots, 5)$ are certain real constants. Then $\langle A, B, C\rangle$ is (non-uniformly) real-diagonalizable if and only if it is equivalent to

$$
\left\langle\left[\begin{array}{lll}
1 & 0 & 0 \\
0 & 0 & 0 \\
0 & 0 & 0
\end{array}\right],\left[\begin{array}{lll}
0 & 1 & 0 \\
1 & 0 & 1 \\
0 & 0 & 0
\end{array}\right],\left[\begin{array}{ccc}
0 & -i & \alpha+i \tilde{\alpha} \\
i & 0 & \beta+i \tilde{\beta} \\
0 & 0 & 0
\end{array}\right]\right\rangle
$$

where $\alpha, \tilde{\alpha}, \beta, \tilde{\beta}$ are arbitrary real constants.

Proof. First notice that, replacing $C$ by $C-\gamma_{1} B$, we may assume

$$
\gamma_{1}=0 \text {. }
$$

Then the succeeding Lemma 5.12 is applicable. Thus we have also 


$$
\tilde{\gamma}_{3}=-\tilde{\gamma}_{1} \neq 0, \quad \gamma_{3}=\gamma_{4}=\tilde{\gamma}_{4}=0 .
$$

So replacing $C$ by $-\tilde{\gamma}_{1}^{-1} C$, we have the conclusion.

Lemma 5.12. Let a nondegenerate matrix family $\langle A, B, C\rangle$ be spanned by

$$
A=\left[\begin{array}{lll}
1 & 0 & 0 \\
0 & 0 & 0 \\
0 & 0 & 0
\end{array}\right], \quad B=\left[\begin{array}{lll}
0 & 1 & 0 \\
1 & 0 & 1 \\
0 & 0 & 0
\end{array}\right], \quad C=\left[\begin{array}{ccc}
0 & i \tilde{\gamma}_{1} & \gamma_{2}+i \tilde{\gamma}_{2} \\
\gamma_{3}+i \tilde{\gamma}_{3} & 0 & \gamma_{5}+i \tilde{\gamma}_{5} \\
\gamma_{4}+i \tilde{\gamma}_{4} & 0 & 0
\end{array}\right] \neq 0
$$

where $\gamma_{j}(j=2, \ldots, 5), \quad \tilde{\gamma}_{j}(j=1, \ldots, 5)$ are certain real constants. Then $\langle A, B, C\rangle$ is (non-uniformly) real-diagonalizable if and only if

$$
\tilde{\gamma}_{3}=-\tilde{\gamma}_{1} \neq 0, \quad \gamma_{3}=\gamma_{4}=\tilde{\gamma}_{4}=0 .
$$

Proof. Taking an arbitrary $\eta \in \mathbb{R}$, the subfamily

$$
\langle A, \eta B+C\rangle=\left\langle\left[\begin{array}{lll}
1 & 0 & 0 \\
0 & 0 & 0 \\
0 & 0 & 0
\end{array}\right],\left[\begin{array}{ccc}
0 & \eta+i \tilde{\gamma}_{1} & \gamma_{2}+i \tilde{\gamma}_{2} \\
\eta+\gamma_{3}+i \tilde{\gamma}_{3} & 0 & \eta+\gamma_{5}+i \tilde{\gamma}_{5} \\
\gamma_{4}+i \tilde{\gamma}_{4} & 0 & 0
\end{array}\right]\right\rangle
$$

is also real-diagonalizable. From Proposition 4.5,

$$
\left(\gamma_{4}+i \tilde{\gamma}_{4}\right)\left(\eta+i \tilde{\gamma}_{1}\right)=0
$$

and

$$
\left(\eta+i \tilde{\gamma}_{1}\right)\left(\eta+\gamma_{3}+i \tilde{\gamma}_{3}\right)+\left(\gamma_{2}+i \tilde{\gamma}_{2}\right)\left(\gamma_{4}+i \tilde{\gamma}_{4}\right)>0
$$

unless $\eta+\gamma_{5}=\tilde{\gamma}_{5}=0$. The first equality implies one of the requirements,

$$
\gamma_{4}=\tilde{\gamma}_{4}=0 \text {. }
$$

Thus the second inequality reduces to

$$
\left(\eta+i \tilde{\gamma}_{1}\right)\left(\eta+\gamma_{3}+i \tilde{\gamma}_{3}\right)>0
$$

unless $\eta+\gamma_{5}=\tilde{\gamma}_{5}=0$. This means also some of the other requirements,

$$
\gamma_{3}=0, \quad \tilde{\gamma}_{3}=-\tilde{\gamma}_{1} .
$$

Now it remains to show $\tilde{\gamma}_{1} \neq 0$. We assume that

$$
\tilde{\gamma}_{1}=0 \text {. }
$$

Because of $\tilde{\gamma}_{3}=-\tilde{\gamma}_{1}$ and $\gamma_{3}=0$ as well as the above $\gamma_{4}=\tilde{\gamma}_{4}=0$, we would obtain

$$
C=\left[\begin{array}{lll}
0 & 0 & * \\
0 & 0 & * \\
0 & 0 & 0
\end{array}\right] \neq 0
$$


where each * is a complex constant. However, this $C$ can not be realdiagonalizable. Thus we are led to a contradiction.

Proposition 5.13. Let a nondegenerate matrix family $\langle A, B, C\rangle$ be spanned by

$$
A=\left[\begin{array}{lll}
1 & 0 & 0 \\
0 & 0 & 0 \\
0 & 0 & 0
\end{array}\right], \quad B=\left[\begin{array}{lll}
0 & 1 & 0 \\
1 & 0 & 1 \\
0 & 0 & 0
\end{array}\right], \quad C=\left[\begin{array}{ccc}
0 & \gamma_{1}+i \tilde{\gamma}_{1} & \gamma_{2}+i \tilde{\gamma}_{2} \\
\gamma_{3}+i \tilde{\gamma}_{3} & 1 & \gamma_{5}+i \tilde{\gamma}_{5} \\
\gamma_{4}+i \tilde{\gamma}_{4} & 0 & -1
\end{array}\right]
$$

where $\gamma_{j}(j=1, \ldots, 5), \tilde{\gamma}_{j}(j=1, \ldots, 5)$ are all real constants. Then $\langle A, B, C\rangle$ is (non-uniformly) real-diagonalizable if and only if it is equivalent to

$$
\left\langle\left[\begin{array}{lll}
1 & 0 & 0 \\
0 & 0 & 0 \\
0 & 0 & 0
\end{array}\right],\left[\begin{array}{lll}
0 & 1 & 0 \\
1 & 0 & 1 \\
0 & 0 & 0
\end{array}\right],\left[\begin{array}{ccc}
0 & \beta-i \delta & -\gamma+\frac{i}{2} \beta \delta \\
\beta(1-\alpha)+i \delta & 1 & i \delta \\
-2 \alpha & 0 & -1
\end{array}\right]\right\rangle
$$

where the real constants satisfy

$$
0<\alpha<1, \quad \gamma>\frac{1}{8}\left(\beta^{2}-4 \delta^{2}\right) .
$$

Proof. First note that, replacing $C$ by $C-\gamma_{5} B$, we may assume

$$
\gamma_{5}=0 \text {. }
$$

Then the following Lemma 5.14 is applicable and we have also

$$
\begin{aligned}
& \tilde{\gamma}_{2}=-\frac{1}{2} \gamma_{1} \tilde{\gamma}_{1}, \quad \gamma_{3}=\frac{1}{2} \gamma_{1}\left(\gamma_{4}+2\right), \quad \tilde{\gamma}_{3}=-\tilde{\gamma}_{1}, \\
& \tilde{\gamma}_{4}=0, \quad \tilde{\gamma}_{5}=-\tilde{\gamma}_{1}, \\
& -2<\gamma_{4}<0, \quad \gamma_{2}<\frac{1}{8}\left\{4 \tilde{\gamma}_{1}^{2}-\gamma_{1}^{2}\right\} .
\end{aligned}
$$

So introducing new parameters $\alpha, \beta, \gamma, \delta$ by

$$
\gamma_{1}=\beta, \quad \tilde{\gamma}_{1}=-\delta, \quad \gamma_{2}=-\gamma, \quad \gamma_{4}=-2 \alpha,
$$

we have the conclusion.

Lemma 5.14. Let a nondegenerate matrix family $\langle A, B, C\rangle$ be spanned by

$$
A=\left[\begin{array}{lll}
1 & 0 & 0 \\
0 & 0 & 0 \\
0 & 0 & 0
\end{array}\right], \quad B=\left[\begin{array}{lll}
0 & 1 & 0 \\
1 & 0 & 1 \\
0 & 0 & 0
\end{array}\right], \quad C=\left[\begin{array}{ccc}
0 & \gamma_{1}+i \tilde{\gamma}_{1} & \gamma_{2}+i \tilde{\gamma}_{2} \\
\gamma_{3}+i \tilde{\gamma}_{3} & 1 & i \tilde{\gamma}_{5} \\
\gamma_{4}+i \tilde{\gamma}_{4} & 0 & -1
\end{array}\right]
$$

where $\gamma_{j}(j=1, \ldots, 4), \tilde{\gamma}_{j}(j=1, \ldots, 5)$ are all real constants. Then $\langle A, B, C\rangle$ is (non-uniformly) real-diagonalizable if and only if 


$$
\begin{aligned}
& \tilde{\gamma}_{2}=-\frac{1}{2} \gamma_{1} \tilde{\gamma}_{1}, \quad \gamma_{3}=\frac{1}{2} \gamma_{1}\left(\gamma_{4}+2\right), \quad \tilde{\gamma}_{3}=-\tilde{\gamma}_{1}, \\
& \tilde{\gamma}_{4}=0, \quad \tilde{\gamma}_{5}=-\tilde{\gamma}_{1}, \\
& -2<\gamma_{4}<0, \quad \gamma_{2}<\frac{1}{8}\left\{4 \tilde{\gamma}_{1}^{2}-\gamma_{1}{ }^{2}\right\} .
\end{aligned}
$$

Proof. Let $\eta \in \mathbb{R}$ be arbitrarily fixed. Consider the similarity transformation for the subfamily $\langle A, \eta B+C\rangle$ with

This leaves

$$
T=\left[\begin{array}{ccc}
1 & 0 & 0 \\
0 & 1 & -\frac{1}{2}\left(\eta+i \tilde{\gamma}_{5}\right) \\
0 & 0 & 1
\end{array}\right]
$$

intact and we also get

$$
\left[\begin{array}{lll}
1 & 0 & 0 \\
0 & 0 & 0 \\
0 & 0 & 0
\end{array}\right]
$$

$$
T^{-1}(\eta B+C) T=\left[\begin{array}{ccc}
0 & \sigma_{1}(\eta)+i \tilde{\sigma}_{1}(\eta) & \sigma_{2}(\eta)+i \tilde{\sigma}_{2}(\eta) \\
\sigma_{3}(\eta)+i \tilde{\sigma}_{3}(\eta) & 1 & 0 \\
\sigma_{4}(\eta)+i \tilde{\sigma}_{4}(\eta) & 0 & -1
\end{array}\right]
$$

where

$$
\begin{aligned}
& \sigma_{1}(\eta) \equiv \eta+\gamma_{1}, \\
& \tilde{\sigma}_{1}(\eta) \equiv \tilde{\gamma}_{1}, \\
& \sigma_{2}(\eta) \equiv-\frac{1}{2}\left\{\eta^{2}+\gamma_{1} \eta-\tilde{\gamma}_{1} \tilde{\gamma}_{5}-2 \gamma_{2}\right\}, \\
& \tilde{\sigma}_{2}(\eta) \equiv-\frac{1}{2}\left\{\left(\tilde{\gamma}_{1}+\tilde{\gamma}_{5}\right) \eta+\gamma_{1} \tilde{\gamma}_{5}-2 \tilde{\gamma}_{2}\right\}, \\
& \sigma_{3}(\eta) \equiv \frac{1}{2}\left\{\left(\gamma_{4}+2\right) \eta-\tilde{\gamma}_{4} \tilde{\gamma}_{5}+2 \gamma_{3}\right\}, \\
& \tilde{\sigma}_{3}(\eta) \equiv \frac{1}{2}\left\{\tilde{\gamma}_{4} \eta+\gamma_{4} \tilde{\gamma}_{5}+2 \tilde{\gamma}_{3}\right\}, \\
& \sigma_{4}(\eta) \equiv \gamma_{4}, \\
& \tilde{\sigma}_{4}(\eta) \equiv \tilde{\gamma}_{4} .
\end{aligned}
$$

By virtue of Proposition 4.3, we can apply Lemma 3.2 to $\sigma_{2}(\eta)+i \tilde{\sigma}_{2}(\eta)$ and $\sigma_{4}(\eta)+i \tilde{\sigma}_{4}(\eta)$. Then $\mu(\eta), \tilde{\mu}(\eta), \psi(\eta)$ must be constants while $\varphi(\eta)$ must be a quadratic. Thus we have $\tilde{\sigma}_{2}(\eta) \equiv 0$ and $\tilde{\sigma}_{4}(\eta) \equiv 0$, that is,

$$
\tilde{\gamma}_{5}=-\tilde{\gamma}_{1}, \quad \tilde{\gamma}_{2}=\frac{1}{2} \gamma_{1} \tilde{\gamma}_{5}=-\frac{1}{2} \gamma_{1} \tilde{\gamma}_{1}, \quad \tilde{\gamma}_{4}=0 .
$$

So $\sigma_{j}(\eta), \tilde{\sigma}_{j}(\eta)(j=1, \ldots, 4)$ are reduced to 


$$
\begin{aligned}
\sigma_{1}(\eta) & \equiv \eta+\gamma_{1} \\
\tilde{\sigma}_{1}(\eta) & \equiv \tilde{\gamma}_{1} \\
\sigma_{2}(\eta) & \equiv-\frac{1}{2}\left\{\eta^{2}+\gamma_{1} \eta+\tilde{\gamma}_{1}^{2}-2 \gamma_{2}\right\} \\
\tilde{\sigma}_{2}(\eta) & \equiv 0 \\
\sigma_{3}(\eta) & \equiv \frac{1}{2}\left\{\left(\gamma_{4}+2\right) \eta+2 \gamma_{3}\right\} \\
\tilde{\sigma}_{3}(\eta) & \equiv \frac{1}{2}\left\{-\tilde{\gamma}_{1} \gamma_{4}+2 \tilde{\gamma}_{3}\right\} \\
\sigma_{4}(\eta) & \equiv \gamma_{4} \\
\tilde{\sigma}_{4}(\eta) & \equiv 0
\end{aligned}
$$

Now, applying Lemma 3.2 to $\sigma_{1}+i \tilde{\sigma}_{1}$ and $\sigma_{3}+i \tilde{\sigma}_{3}$, we have or equivalently

$$
\sigma_{3}(\eta) \equiv \frac{1}{2}\left(\gamma_{4}+2\right) \sigma_{1}(\eta), \quad \tilde{\sigma}_{3}(\eta) \equiv-\frac{1}{2}\left(\gamma_{4}+2\right) \tilde{\sigma}_{1}(\eta)
$$

$$
\gamma_{3}=\frac{1}{2} \gamma_{1}\left(\gamma_{4}+2\right), \quad \tilde{\gamma}_{3}=-\tilde{\gamma}_{1}
$$

So $\sigma_{j}(\eta), \tilde{\sigma}_{j}(\eta)(j=1, \ldots, 4)$ are reduced to

$$
\begin{aligned}
\sigma_{1}(\eta) & \equiv \eta+\gamma_{1} \\
\tilde{\sigma}_{1}(\eta) & \equiv \tilde{\gamma}_{1} \\
\sigma_{2}(\eta) & \equiv-\frac{1}{2}\left\{\eta^{2}+\gamma_{1} \eta+\tilde{\gamma}_{1}^{2}-2 \gamma_{2}\right\} \\
\tilde{\sigma}_{2}(\eta) & \equiv 0 \\
\sigma_{3}(\eta) & \equiv \frac{1}{2}\left(\gamma_{4}+2\right)\left(\eta+\gamma_{1}\right) \\
\tilde{\sigma}_{3}(\eta) & \equiv-\frac{1}{2} \tilde{\gamma}_{1}\left(\gamma_{4}+2\right) \\
\sigma_{4}(\eta) & \equiv \gamma_{4} \\
\tilde{\sigma}_{4}(\eta) & \equiv 0
\end{aligned}
$$

So applying Lemma 3.2 again, we know that $\sigma_{2}(\eta)$ is negative definite and that

$$
\gamma_{4}<0, \quad \gamma_{4}>-2 \text {. }
$$

The first condition is equivalent to

$$
4\left(\tilde{\gamma}_{1}^{2}-2 \gamma_{2}\right)-\gamma_{1}^{2}>0
$$

namely,

$$
\gamma_{2}<\frac{1}{8}\left\{4 \tilde{\gamma}_{1}^{2}-\gamma_{1}^{2}\right\} .
$$

The converse is clear from the above calculations. 
Let us write down the summary of this section as a theorem.

Theorem 5.15. Let $\left\langle A_{1}, A_{2}, A_{3}\right\rangle$ be a nondegenerate $3 \times 3$ matrix family with multiple eigenvalues. Then the following 1) and 2) hold.

1) Suppose $\left\langle A_{1}, A_{2}, A_{3}\right\rangle$ is uniformly real-diagonalizable and is not equivalent to any hermitian family. Then $\left\langle A_{1}, A_{2}, A_{3}\right\rangle$ is equivalent either to

$$
\left\langle\left[\begin{array}{lll}
1 & 0 & 0 \\
0 & 0 & 0 \\
0 & 0 & 0
\end{array}\right],\left[\begin{array}{lll}
0 & 1 & 0 \\
1 & 0 & 0 \\
0 & 0 & 0
\end{array}\right],\left[\begin{array}{ccc}
0 & -c+\frac{1}{c} & c+\frac{1}{c} \\
c-\frac{1}{c} & 0 & 0 \\
c+\frac{1}{c} & 0 & 0
\end{array}\right]\right\rangle
$$

where $c \neq 0$ is an arbitrary complex constant, or to

$$
\left\langle\left[\begin{array}{lll}
1 & 0 & 0 \\
0 & 0 & 0 \\
0 & 0 & 0
\end{array}\right],\left[\begin{array}{lll}
0 & 1 & 0 \\
1 & 0 & 0 \\
0 & 0 & 0
\end{array}\right],\left[\begin{array}{rrr}
0 & -i & -i \\
i & 0 & 0 \\
0 & 0 & 0
\end{array}\right]\right\rangle
$$

or to its transpose,

$$
\left\langle\left[\begin{array}{lll}
1 & 0 & 0 \\
0 & 0 & 0 \\
0 & 0 & 0
\end{array}\right],\left[\begin{array}{lll}
0 & 1 & 0 \\
1 & 0 & 0 \\
0 & 0 & 0
\end{array}\right],\left[\begin{array}{rrr}
0 & -i & 0 \\
i & 0 & 0 \\
i & 0 & 0
\end{array}\right]\right\rangle
$$

or

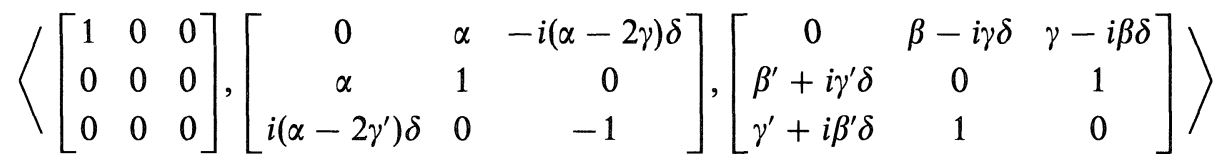

where the real constants $\alpha, \beta, \beta^{\prime}, \gamma, \gamma^{\prime}$ and $\delta$ satisfy

$$
\begin{gathered}
\alpha>0, \quad \gamma>\frac{1}{2}\left(\alpha+\frac{\beta^{2}}{\alpha}\right), \quad \gamma^{\prime}>\frac{1}{2}\left(\alpha+\frac{\beta^{2}}{\alpha}\right), \\
\left|\beta-\beta^{\prime}\right|+\left|\gamma-\gamma^{\prime}\right|>0 .
\end{gathered}
$$

2) Suppose now that $\langle A, B, C\rangle$ is non-uniformly real-diagonalizable. Then $\langle A, B, C\rangle$ is equivalent to either

$$
\left\langle\left[\begin{array}{lll}
1 & 0 & 0 \\
0 & 0 & 0 \\
0 & 0 & 0
\end{array}\right],\left[\begin{array}{lll}
0 & 1 & 0 \\
1 & 0 & 1 \\
0 & 0 & 0
\end{array}\right],\left[\begin{array}{rrr}
0 & -i & c_{1} \\
i & 0 & c_{2} \\
0 & 0 & 0
\end{array}\right]\right\rangle
$$

where $c_{1}, c_{2}$ are arbitrary complex constants, or 


$$
\left\langle\left[\begin{array}{lll}
1 & 0 & 0 \\
0 & 0 & 0 \\
0 & 0 & 0
\end{array}\right],\left[\begin{array}{lll}
0 & 1 & 0 \\
1 & 0 & 1 \\
0 & 0 & 0
\end{array}\right],\left[\begin{array}{ccc}
0 & \beta-i \delta & -\gamma+\frac{i}{2} \beta \delta \\
\beta(1-\alpha)+i \delta & 1 & i \delta \\
-2 \alpha & 0 & -1
\end{array}\right]\right\rangle
$$

where the real constants $\alpha, \beta, \gamma$ and $\delta$ satisfy

$$
0<\alpha<1, \quad \gamma>\frac{1}{8}\left(\beta^{2}-4 \delta^{2}\right)
$$

or their transposes.

\section{§6. Families Spanned by Four Matrices}

Let us begin with matrix families of the form:

$$
\left\langle\left[\begin{array}{lll}
1 & 0 & 0 \\
0 & 0 & 0 \\
0 & 0 & 0
\end{array}\right],\left[\begin{array}{lll}
0 & * & * \\
* & 0 & 0 \\
* & 0 & 0
\end{array}\right], \ldots\right\rangle
$$

where each * stands for a complex constant.

Proposition 6.1. Let a nondegenerate matrix family $\left\langle A, B_{1}, B_{2}, B_{3}\right\rangle$ be spanned by

$$
A=\left[\begin{array}{lll}
1 & 0 & 0 \\
0 & 0 & 0 \\
0 & 0 & 0
\end{array}\right], \quad B_{k}=\left[\begin{array}{ccc}
0 & b_{k 1} & b_{k 2} \\
b_{k 3} & 0 & 0 \\
b_{k 4} & 0 & 0
\end{array}\right],
$$

where $b_{k j}(k=1,2,3 ; j=1,2,3,4)$ are complex constants. Then $\left\langle A, B_{1}, B_{2}, B_{3}\right\rangle$ is real-diagonalizable if and only if it is equivalent to either

$\left\langle\left[\begin{array}{lll}1 & 0 & 0 \\ 0 & 0 & 0 \\ 0 & 0 & 0\end{array}\right],\left[\begin{array}{lll}0 & 1 & 0 \\ 1 & 0 & 0 \\ 0 & 0 & 0\end{array}\right],\left[\begin{array}{lll}0 & 0 & 1 \\ 0 & 0 & 0 \\ 1 & 0 & 0\end{array}\right],\left[\begin{array}{ccc}0 & -c+\frac{1}{c} & -i\left(c+\frac{1}{c}\right) \\ c-\frac{1}{c} & 0 & 0 \\ i\left(c+\frac{1}{c}\right) & 0 & 0\end{array}\right]\right\rangle$

with $c \neq 0$ an arbitrary complex constant, or

$\left\langle\left[\begin{array}{lll}1 & 0 & 0 \\ 0 & 0 & 0 \\ 0 & 0 & 0\end{array}\right],\left[\begin{array}{lll}0 & 1 & 0 \\ 1 & 0 & 0 \\ 0 & 0 & 0\end{array}\right],\left[\begin{array}{ccc}0 & -\alpha & 1 \\ \alpha & 0 & 0 \\ 1 & 0 & 0\end{array}\right],\left[\begin{array}{ccc}0 & -i(1+\gamma)\left(\beta+i \frac{\alpha}{\gamma}\right) \\ i & 0 & 0 \\ (1-\gamma)\left(\beta-i \frac{\alpha}{\gamma}\right) & 0 & 0\end{array}\right]\right\rangle$

where $0<\alpha<1, \beta \neq 0, \gamma \neq 0$ are real constants satisfying $\beta^{2} \gamma^{2}<1-\alpha^{2}$, or 


$$
\left\langle\left[\begin{array}{lll}
1 & 0 & 0 \\
0 & 0 & 0 \\
0 & 0 & 0
\end{array}\right],\left[\begin{array}{lll}
0 & 1 & 0 \\
1 & 0 & 0 \\
0 & 0 & 0
\end{array}\right],\left[\begin{array}{ccc}
0 & -\alpha & 1 \\
\alpha & 0 & 0 \\
1 & 0 & 0
\end{array}\right],\left[\begin{array}{ccc}
0 & -i & i(\alpha+\gamma) \\
i & 0 & 0 \\
i(\alpha-\gamma) & 0 & 0
\end{array}\right]\right\rangle
$$

where $0<\alpha<1$ and $\gamma$ are real constant, or

$$
\left\langle\left[\begin{array}{lll}
1 & 0 & 0 \\
0 & 0 & 0 \\
0 & 0 & 0
\end{array}\right],\left[\begin{array}{lll}
0 & 1 & 0 \\
1 & 0 & 0 \\
0 & 0 & 0
\end{array}\right],\left[\begin{array}{ccc}
0 & -\alpha & 1 \\
\alpha & 0 & 0 \\
1 & 0 & 0
\end{array}\right],\left[\begin{array}{ccc}
0 & -i & (i \alpha+\gamma) \\
i & 0 & 0 \\
(i \alpha-\gamma) & 0 & 0
\end{array}\right]\right\rangle
$$

where $\alpha$ and $\gamma$ are real constants satisfying $0<\alpha<1$ and $\alpha^{2}+\gamma^{2}<1$.

Moreover, in all of these cases, $\left\langle A, B_{1}, B_{2}, B_{3}\right\rangle$ is uniformly real-diagonalizable.

Proof. By Propositions 4.1 and 4.2, we may specify

$$
B_{1}=\left[\begin{array}{lll}
0 & 1 & 0 \\
1 & 0 & 0 \\
0 & 0 & 0
\end{array}\right]
$$

Hence, by the same argument as in the proof of Proposition 5.1, we may also assume

$$
b_{k 1}+b_{k 3}=0 \quad(k=2,3) .
$$

By a suitable change of basis, we may further assume either

$$
b_{21}\left(=b_{23}\right)=0
$$

or

$$
b_{21}=-1 \quad\left(b_{23}=1\right), \quad b_{31}=-i \quad\left(b_{33}=i\right) .
$$

Let us begin with the first case. Applying Proposition 4.1 to $\left\langle A, B_{2}\right\rangle$, we have

$$
b_{22} b_{24}>0 \text {. }
$$

So, after using the similarity transformation with

$$
T=\left[\begin{array}{lll}
1 & 0 & 0 \\
0 & 1 & 0 \\
0 & 0 & d
\end{array}\right] \quad(d: \text { complex })
$$

we may assume $b_{22}=b_{24}=1$ (recall also $b_{21}=b_{23}=0$ ), that is,

$$
B_{2}=\left[\begin{array}{lll}
0 & 0 & 1 \\
0 & 0 & 0 \\
1 & 0 & 0
\end{array}\right]
$$


Because $\left\langle A, \eta B_{2}+B_{4}\right\rangle\left(\eta\right.$ : arbitrarily fixed) is real-diagonalizable, $b_{32}+b_{34}$ must be real. Replacing $B_{3}$ by

$$
B_{3}-\frac{1}{2}\left(b_{32}+b_{34}\right) B_{2}
$$

we may further assume

$$
b_{32}=-b_{34}
$$

as well as $b_{31}=-b_{33}$. On the other hand, a necessary and sufficient condition for $\left\langle A, B_{1}, B_{2}, B_{3}\right\rangle$ to be real-diagonalizable is that

$$
\left\langle A, \eta_{1} B_{1}+\eta_{2} B_{2}+\eta_{3} B_{3}\right\rangle
$$

is real-diagonalizable for any $\left(\eta_{1}, \eta_{2}, \eta_{3}\right) \in \mathbb{R}^{3}$. By virtue of Proposition 4.1, the last condition is equivalent to

$$
b_{31} b_{33}+b_{32} b_{34}=-b_{33}{ }^{2}-b_{34}{ }^{2}>0 \text {. }
$$

Replacing $B_{3}$ by its suitable real scalar multiple, we may assume

$$
-b_{33}{ }^{2}-b_{34}^{2}=4 \text {. }
$$

So putting

$$
b_{33}=c-\frac{1}{c}
$$

we have

$$
b_{34}= \pm i\left(c+\frac{1}{c}\right) .
$$

Here the minus sign can be excluded by the similarity transformation with

$$
T=\left[\begin{array}{rrr}
1 & 0 & 0 \\
0 & 1 & 0 \\
0 & 0 & -1
\end{array}\right]
$$

Thus we have obtained the first family of the requirement.

Let us now consider the second case. Applying Proposition 4.1 to $\left\langle A, B_{2}\right\rangle$, we know

$$
b_{21} b_{23}+b_{22} b_{24}=-1+b_{22} b_{24}>0 .
$$

So using a similarity transformation with

$$
T=\left[\begin{array}{lll}
1 & 0 & 0 \\
0 & 1 & 0 \\
0 & 0 & d
\end{array}\right] \quad(d \neq 0: \text { complex })
$$


we can assume that $b_{22}=b_{24}>0$. Thus we may assume

$$
B_{2}=\left[\begin{array}{ccc}
0 & -\alpha & 1 \\
\alpha & 0 & 0 \\
1 & 0 & 0
\end{array}\right] \quad(0<\alpha<1),
$$

replacing $B_{2}$ by its suitable real scalar multiple. Let us apply Proposition 4.1 to $\left\langle A, \xi B_{2}+\eta B_{3}\right\rangle$ with $(\xi, \eta) \in \mathbb{R}^{2} \backslash\{(0,0)\}$ arbitrarily fixed. Thus it suffices to find the condition that

$$
\left(1-\alpha^{2}\right) \xi^{2}+\left(b_{32}+b_{34}-2 i \alpha\right) \xi \eta+\left(1+b_{32} b_{34}\right) \eta^{2}
$$

is real and positive for all $(\xi, \eta) \in \mathbb{R}^{2} \backslash\{(0,0)\}$. Since $b_{32}+b_{34}-2 i \alpha$ is real, we may put

$$
b_{32}=c+i \alpha+\beta, \quad b_{34}=-c+i \alpha+\beta
$$

where $c$ is complex and $\beta$ is real. Thus (6.9) becomes

$$
\left(1-\alpha^{2}\right) \xi^{2}+2 \beta \xi \eta+\left\{1-c^{2}+(i \alpha+\beta)^{2}\right\} \eta^{2} .
$$

We split the case according to $\beta \neq 0$ or $\beta=0$. Let us begin with the case $\beta \neq 0$. Since $(i \alpha+\beta)^{2}-c^{2}$ is real, we may put

$$
c=i \frac{\alpha}{\gamma}+\beta \gamma
$$

for some real $\gamma \neq 0$. The quadratic form (6.11) then becomes

$$
\left(1-\alpha^{2}\right) \xi^{2}+2 \beta \xi \eta+\left\{\frac{\alpha^{2}}{\gamma^{2}}-\alpha^{2}+\beta^{2}-\beta^{2} \gamma^{2}+1\right\} \eta^{2} .
$$

Now, one quarter of the discriminant of this quadratic form is

$$
\beta^{2}-\left(1-\alpha^{2}\right)\left\{\frac{\alpha^{2}}{\gamma^{2}}-\alpha^{2}+\beta^{2}-\beta^{2} \gamma^{2}+1\right\}=\left(\frac{\alpha^{2}}{\gamma^{2}}+1-\alpha^{2}\right)\left\{\beta^{2} \gamma^{2}-\left(1-\alpha^{2}\right)\right\} .
$$

So our condition is, (recall $0<\alpha<1$ ),

$$
\beta^{2} \gamma^{2}<1-\alpha^{2}
$$

Let us turn to the case $\beta=0$ in (6.11). This now becomes

$$
\left(1-\alpha^{2}\right) \xi^{2}+\left(1-c^{2}-\alpha^{2}\right) \eta^{2} \text {. }
$$

This quadratic form is positive definite if and only if $c$ is a pure imaginary or is a real satisfying

$$
c^{2}+\alpha^{2}<1 .
$$

Putting $c=i \gamma$ or $c=\gamma$, we obtain the last two families of the requirement. 
Finally, to prove the uniform real-diagonalizability, we need only repeat the same procedure as in the proof of Proposition 5.1.

Most of the matrix families indicated in Proposition 6.1 are not equivalent to any hermitian family as will be shown next.

Proposition 6.2. Neither of the following 1), 2), 3), 4) is equivalent to any hermitian family.

1)

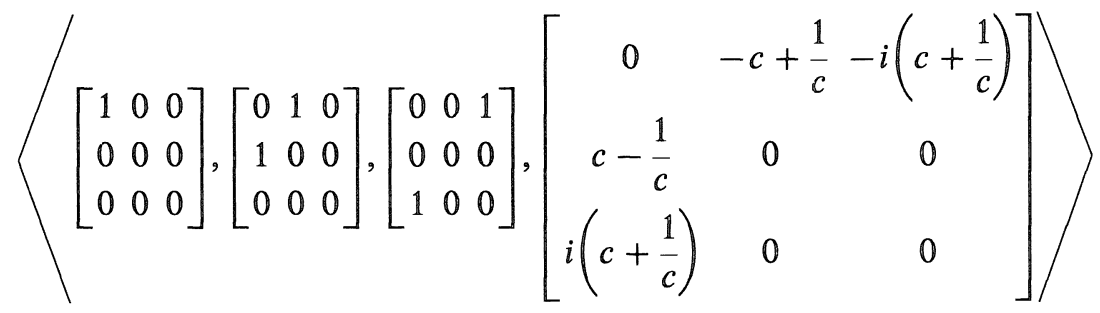

with an arbitrary complex constant $c \neq 0$ satisfying $|c| \neq 1$.

2)

$$
\left\langle\left[\begin{array}{lll}
1 & 0 & 0 \\
0 & 0 & 0 \\
0 & 0 & 0
\end{array}\right],\left[\begin{array}{lll}
0 & 1 & 0 \\
1 & 0 & 0 \\
0 & 0 & 0
\end{array}\right],\left[\begin{array}{ccc}
0 & -\alpha & 1 \\
\alpha & 0 & 0 \\
1 & 0 & 0
\end{array}\right],\left[\begin{array}{ccc}
0 & -i(1+\gamma)\left(\beta+i \frac{\alpha}{\gamma}\right) \\
i & 0 & 0 \\
(1-\gamma)\left(\beta-i \frac{\alpha}{\gamma}\right) & 0 & 0
\end{array}\right]\right\rangle
$$

where $0<\alpha<1, \beta \neq 0, \gamma \neq 0$ are real constants satisfying $\beta^{2} \gamma^{2}<1-\alpha^{2}$.

3) $\left\langle\left[\begin{array}{lll}1 & 0 & 0 \\ 0 & 0 & 0 \\ 0 & 0 & 0\end{array}\right],\left[\begin{array}{lll}0 & 1 & 0 \\ 1 & 0 & 0 \\ 0 & 0 & 0\end{array}\right],\left[\begin{array}{ccc}0 & -\alpha & 1 \\ \alpha & 0 & 0 \\ 1 & 0 & 0\end{array}\right],\left[\begin{array}{ccc}0 & -i & i(\alpha+\gamma) \\ i & 0 & 0 \\ i(\alpha-\gamma) & 0 & 0\end{array}\right]\right\rangle$

where $0<\alpha<1$ and $\gamma$ are real constants.

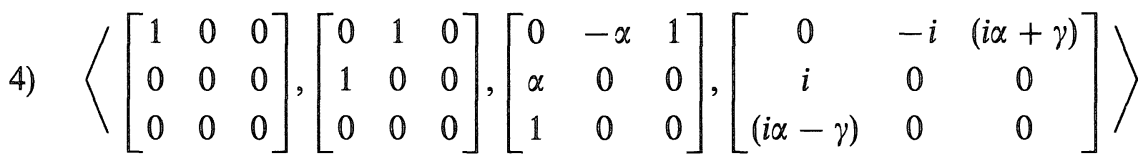

where $\alpha$ and $\gamma$ are real constants satisfying $0<\alpha<1$ and $\alpha^{2}+\gamma^{2}<1$.

Proof. Let us begin with the first family. Denote the matrices by $A, B_{1}$, $B_{2}, B_{3}$. Assume, to the contrary, that there exists a nonsingular matrix $T$ such that

$$
T^{-1} A T, \quad T^{-1} B_{1} T, \quad T^{-1} B_{2} T, \quad T^{-1} B_{3} T
$$

are simultaneously hermitian. By the same argument as in the proof of Lemma 5.2 , we may assume 


$$
T=\left[\begin{array}{lll}
1 & 0 & 0 \\
0 & 1 & 0 \\
0 & 0 & d
\end{array}\right] \quad(d \neq 0: \text { complex })
$$

Since the $(1,3)$ - and $(3,1)$ - entries of $T^{-1} B_{2} T$ are complex conjugate, we have $\bar{d}=1 / d$, that is, $|d|=1$. Thus $T$ is unitary and (6.15) means that

$$
A, \quad B_{1}, \quad B_{2}, \quad B_{3}
$$

are hermitian. However, $B_{3}$ is not hermitian because $|c| \neq 1$. We are thus led to a contradiction.

The same argument is valid for the families 2), 3), 4). So we omit the proof for these cases.

Let us turn to the other types of matrix families. In Proposition 5.3, we have already proved that any real-diagonalizable matrix family of the form:

$$
\left\langle\left[\begin{array}{lll}
1 & 0 & 0 \\
0 & 0 & 0 \\
0 & 0 & 0
\end{array}\right],\left[\begin{array}{rrr}
0 & * & * \\
* & 1 & 0 \\
* & 0 & -1
\end{array}\right],\left[\begin{array}{rrr}
0 & * & * \\
* & 0 & 0 \\
* & 0 & 0
\end{array}\right], \ldots\right\rangle,
$$

is equivalent to a hermitian family. We will prove that the real-diagonalizable family of the form:

$$
\left\langle\left[\begin{array}{lll}
1 & 0 & 0 \\
0 & 0 & 0 \\
0 & 0 & 0
\end{array}\right],\left[\begin{array}{rrr}
0 & * & * \\
* & 1 & 0 \\
* & 0 & -1
\end{array}\right],\left[\begin{array}{rrr}
0 & * & * \\
* & 0 & 1 \\
* & 1 & 0
\end{array}\right],\left[\begin{array}{rrr}
0 & * & * \\
* & 0 & 0 \\
* & 0 & 0
\end{array}\right], \ldots\right\rangle,
$$

is also equivalent to a hermitian family.

Proposition 6.3. Let a nondegenerate matrix family $\left\langle A, B_{1}, B_{2}, C_{1}, \ldots, C_{n}\right\rangle$ $(n \geq 1)$ be spanned by

$$
\begin{aligned}
A & =\left[\begin{array}{lll}
1 & 0 & 0 \\
0 & 0 & 0 \\
0 & 0 & 0
\end{array}\right], \\
B_{1} & =\left[\begin{array}{ccc}
0 & \beta_{11}+i \tilde{\beta}_{11} & \beta_{12}+i \tilde{\beta}_{12} \\
\beta_{13}+i \tilde{\beta}_{13} & 1 & 0 \\
\beta_{14}+i \tilde{\beta}_{14} & 0 & -1
\end{array}\right], \\
B_{2} & =\left[\begin{array}{ccc}
0 & \beta_{21}+i \tilde{\beta}_{21} & \beta_{22}+i \tilde{\beta}_{22} \\
\beta_{23}+i \tilde{\beta}_{23} & 0 & 1 \\
\beta_{24}+i \tilde{\beta}_{24} & 1 & 0
\end{array}\right], \\
C_{j} & =\left[\begin{array}{ccc}
0 & \gamma_{j 1}+i \tilde{\gamma}_{j 1} & \gamma_{j 2}+i \tilde{\gamma}_{j 2} \\
\gamma_{j 3}+i \tilde{\gamma}_{j 3} & 0 & 0 \\
\gamma_{j 4}+i \tilde{\gamma}_{j 4} & 0 & 0
\end{array}\right],
\end{aligned}
$$


where $\beta_{j k}, \tilde{\beta}_{j k}, \gamma_{j k}, \tilde{\gamma}_{j k}$ are arbitrary real constants. If $\left\langle A, B_{1}, B_{2}, C_{1}, \ldots, C_{n}\right\rangle$ is real-diagonalizable, then it is equivalent to a hermitian family.

Proof. Consider

$$
\begin{aligned}
& \left(\eta_{1}{ }^{2}-\eta_{2}{ }^{2}\right) B_{1}+2 \eta_{1} \eta_{2} B_{2}+\zeta_{1} C_{1}+\cdots+\zeta_{n} C_{n} \\
& \quad \equiv\left[\begin{array}{ccc}
0 & \rho_{1}(\eta, \zeta)+i \tilde{\rho}_{1}(\eta, \zeta) & \rho_{2}(\eta, \zeta)+i \tilde{\rho}_{2}(\eta, \zeta) \\
\rho_{3}(\eta, \zeta)+i \tilde{\rho}_{3}(\eta, \zeta) & \eta_{1}{ }^{2}-\eta_{2}{ }^{2} & 2 \eta_{1} \eta_{2} \\
\rho_{4}(\eta, \zeta)+i \tilde{\rho}_{4}(\eta, \zeta) & 2 \eta_{1} \eta_{2} & -\eta_{1}{ }^{2}+\eta_{2}{ }^{2}
\end{array}\right]
\end{aligned}
$$

with any fixed $(\eta, \zeta) \in \mathbb{R}^{2+n} \backslash\{(0,0)\}$. Here

$$
\begin{gathered}
\rho_{k}(\eta, \zeta) \equiv \beta_{1 k}\left(\eta_{1}{ }^{2}-\eta_{2}{ }^{2}\right)+2 \beta_{2 k} \eta_{1} \eta_{2}+\sum_{j=1}^{n} \gamma_{j k} \zeta_{j}, \\
\tilde{\rho}_{k}(\eta, \zeta) \equiv \tilde{\beta}_{1 k}\left(\eta_{1}{ }^{2}-\eta_{2}{ }^{2}\right)+2 \tilde{\beta}_{2 k} \eta_{1} \eta_{2}+\sum_{j=1}^{n} \tilde{\gamma}_{j k} \zeta_{j}
\end{gathered}
$$

for $k=1,2,3,4$. Note that

$$
\left\langle A,\left(\eta_{1}{ }^{2}-\eta_{2}{ }^{2}\right) B_{1}+2 \eta_{1} \eta_{2} B_{2}+\sum \zeta_{k} C_{k}\right\rangle \subset\left\langle A, B_{1}, B_{2}, C_{1}, \ldots, C_{n}\right\rangle
$$

is clearly real-diagonalizable. Define a real orthogonal matrix $V(\eta)$ by

$$
V(\eta) \equiv\left[\begin{array}{ccc}
1 & 0 & 0 \\
0 & \eta_{1} /\|\eta\| & -\eta_{2} /\|\eta\| \\
0 & \eta_{2} /\|\eta\| & \eta_{1} /\|\eta\|
\end{array}\right]
$$

where

$$
\|\eta\|=\left(\eta_{1}{ }^{2}+\eta_{2}{ }^{2}\right)^{1 / 2} .
$$

By the similarity transformation with this $V(\eta)$,

$$
\left\langle A,\left(\eta_{1}^{2}-\eta_{2}^{2}\right) B_{1}+2 \eta_{1} \eta_{2} B_{2}+\sum \zeta_{k} C_{k}\right\rangle
$$

is equivalent to

$\left\langle\left[\begin{array}{lll}1 & 0 & 0 \\ 0 & 0 & 0 \\ 0 & 0 & 0\end{array}\right],\|\eta\|^{-1} \cdot\left[\begin{array}{ccc}0 & \sigma_{1}(\eta, \zeta)+i \tilde{\sigma}_{1}(\eta, \zeta) & \sigma_{2}(\eta, \zeta)+i \tilde{\sigma}_{2}(\eta, \zeta) \\ \sigma_{3}(\eta, \zeta)+i \tilde{\sigma}_{3}(\eta, \zeta) & \|\eta\|^{3} & 0 \\ \sigma_{4}(\eta, \zeta)+i \tilde{\sigma}_{4}(\eta, \zeta) & 0 & -\|\eta\|^{3}\end{array}\right]\right\rangle$

where

$$
\begin{aligned}
\sigma_{1}(\eta, \zeta) \equiv & \left(\eta_{1}^{2}-\eta_{2}^{2}\right)\left(\beta_{11} \eta_{1}+\beta_{12} \eta_{2}\right)+2 \eta_{1} \eta_{2}\left(\beta_{21} \eta_{1}+\beta_{22} \eta_{2}\right) \\
& +\sum_{j=1}^{n}\left(\gamma_{j 1} \eta_{1}+\gamma_{j 2} \eta_{2}\right) \zeta_{j} \\
\tilde{\sigma}_{1}(\eta, \zeta) \equiv & \left(\eta_{1}^{2}-\eta_{2}^{2}\right)\left(\tilde{\beta}_{11} \eta_{1}+\tilde{\beta}_{12} \eta_{2}\right)+2 \eta_{1} \eta_{2}\left(\tilde{\beta}_{21} \eta_{1}+\tilde{\beta}_{22} \eta_{2}\right) \\
& +\sum_{j=1}^{n}\left(\tilde{\gamma}_{j 1} \eta_{1}+\tilde{\gamma}_{j 2} \eta_{2}\right) \zeta_{j}
\end{aligned}
$$




$$
\begin{aligned}
\sigma_{2}(\eta, \zeta) \equiv & \left(\eta_{1}{ }^{2}-\eta_{2}{ }^{2}\right)\left(\beta_{12} \eta_{1}+\beta_{11} \eta_{2}\right)+2 \eta_{1} \eta_{2}\left(\beta_{22} \eta_{1}-\beta_{21} \eta_{2}\right) \\
& +\sum_{j=1}^{n}\left(\gamma_{j 2} \eta_{1}-\gamma_{j 1} \eta_{2}\right) \zeta_{j}, \\
\tilde{\sigma}_{2}(\eta, \zeta) \equiv & \left(\eta_{1}{ }^{2}-\eta_{2}{ }^{2}\right)\left(\tilde{\beta}_{12} \eta_{1}+\tilde{\beta}_{11} \eta_{2}\right)+2 \eta_{1} \eta_{2}\left(\tilde{\beta}_{22} \eta_{1}-\tilde{\beta}_{21} \eta_{2}\right) \\
& +\sum_{j=1}^{n}\left(\tilde{\gamma}_{j 2} \eta_{1}-\tilde{\gamma}_{j 1} \eta_{2}\right) \zeta_{j}, \\
\sigma_{3}(\eta, \zeta) \equiv & \left(\eta_{1}{ }^{2}-\eta_{2}{ }^{2}\right)\left(\beta_{13} \eta_{1}+\beta_{14} \eta_{2}\right)+2 \eta_{1} \eta_{2}\left(\beta_{23} \eta_{1}+\beta_{24} \eta_{2}\right) \\
& +\sum_{j=1}^{n}\left(\gamma_{j 3} \eta_{1}+\gamma_{j 4} \eta_{2}\right) \zeta_{j}, \\
\tilde{\sigma}_{3}(\eta, \zeta) \equiv & \left(\eta_{1}{ }^{2}-\eta_{2}{ }^{2}\right)\left(\tilde{\beta}_{13} \eta_{1}+\tilde{\beta}_{14} \eta_{2}\right)+2 \eta_{1} \eta_{2}\left(\tilde{\beta}_{23} \eta_{1}+\tilde{\beta}_{24} \eta_{2}\right) \\
& +\sum_{j=1}^{n}\left(\tilde{\gamma}_{j 3} \eta_{1}+\tilde{\gamma}_{j 4} \eta_{2}\right) \zeta_{j}, \\
\sigma_{4}(\eta, \zeta) \equiv & \left(\eta_{1}{ }^{2}-\eta_{2}{ }^{2}\right)\left(\beta_{14} \eta_{1}-\beta_{13} \eta_{2}\right)+2 \eta_{1} \eta_{2}\left(\beta_{24} \eta_{1}-\beta_{23} \eta_{2}\right) \\
& +\sum_{j=1}^{n}\left(\gamma_{j 4} \eta_{1}-\gamma_{j 3} \eta_{2}\right) \zeta_{j}, \\
\tilde{\sigma}_{4}(\eta, \zeta) \equiv & \left(\eta_{1}{ }^{2}-\eta_{2}{ }^{2}\right)\left(\tilde{\beta}_{14} \eta_{1}-\tilde{\beta}_{13} \eta_{2}\right)+2 \eta_{1} \eta_{2}\left(\tilde{\beta}_{24} \eta_{1}-\tilde{\beta}_{23} \eta_{2}\right) \\
& +\sum_{j=1}^{n}\left(\tilde{\gamma}_{j 4} \eta_{1}-\tilde{\gamma}_{j 3} \eta_{2}\right) \zeta_{j} .
\end{aligned}
$$

Note that all of $\sigma_{k}(\eta, \zeta)$ and $\tilde{\sigma}_{k}(\eta, \zeta)(k=1, \ldots, 4)$ are linear polynomials with respect to $\zeta=\left(\zeta_{1}, \ldots, \zeta_{n}\right)$ and each of the coefficients of $\zeta_{k}(k=1, \ldots, n)$ is a linear polynomials in $\eta=\left(\eta_{1}, \eta_{2}\right)$. Let us apply Proposition 4.3 and Lemma 3.2 to

$$
\sigma_{1}(\eta, \zeta)+i \tilde{\sigma}_{1}(\eta, \zeta)
$$

and

$$
\sigma_{3}(\eta, \zeta)+i \tilde{\sigma}_{3}(\eta, \zeta)
$$

Thus we have

$$
\begin{aligned}
& \operatorname{sgn} \sigma_{1}(\eta, \zeta) \equiv \operatorname{sgn} \sigma_{3}(\eta, \zeta), \\
& \operatorname{sgn} \tilde{\sigma}_{1}(\eta, \zeta) \equiv-\operatorname{sgn} \tilde{\sigma}_{3}(\eta, \zeta) .
\end{aligned}
$$

Recall that $\sigma_{j}(\eta, \zeta)$ and $\tilde{\sigma}_{j}(\eta, \zeta)$ are linear with respect to $\zeta$ and their coefficients of $\zeta$ are linear polynomials in $\eta$. Consequently there exist positive constants $\alpha>0$ and $\tilde{\alpha}>0$ such that

Meanwhile

$$
\sigma_{3}(\eta, \zeta) \equiv \alpha \sigma_{1}(\eta, \zeta), \quad \tilde{\sigma}_{3}(\eta, \zeta) \equiv-\tilde{\alpha} \tilde{\sigma}_{1}(\eta, \zeta)
$$

$$
\begin{aligned}
& \left\{\sigma_{1}(\eta, \zeta)+i \tilde{\sigma}_{1}(\eta, \zeta)\right\}\left\{\sigma_{3}(\eta, \zeta)+i \tilde{\sigma}_{3}(\eta, \zeta)\right\} \\
& \quad \equiv\left\{\sigma_{1}(\eta, \zeta)+i \tilde{\sigma}_{1}(\eta, \zeta)\right\}\left\{\alpha \sigma_{1}(\eta, \zeta)-i \tilde{\alpha} \tilde{\sigma}_{1}(\eta, \zeta)\right\} \\
& \quad \equiv \alpha\left\{\sigma_{1}(\eta, \zeta)\right\}^{2}+\tilde{\alpha}\left\{\tilde{\sigma}_{1}(\eta, \zeta)\right\}^{2}+i(\alpha-\tilde{\alpha}) \sigma_{1}(\eta, \zeta) \tilde{\sigma}_{1}(\eta, \zeta)
\end{aligned}
$$


must be real for all $(\eta, \zeta) \in \mathbb{R}^{2+n}$. Thus we obtain $\tilde{\alpha}=\alpha>0$, that is,

$$
\sigma_{3}(\eta, \zeta) \equiv \alpha \sigma_{1}(\eta, \zeta), \quad \tilde{\sigma}_{3}(\eta, \zeta) \equiv-\alpha \tilde{\sigma}_{1}(\eta, \zeta) \quad(\alpha>0) .
$$

Hence, these identities imply

$$
\beta_{j 3}=\alpha \beta_{j 1}, \quad \beta_{j 4}=\alpha \beta_{j 2}, \quad \tilde{\beta}_{j 3}=-\alpha \tilde{\beta}_{j 1}, \quad \tilde{\beta}_{j 4}=-\alpha \tilde{\beta}_{j 2},
$$

for $j=1,2$ and

$$
\gamma_{k 3}=\alpha \gamma_{k 1}, \quad \gamma_{k 4}=\alpha \gamma_{k 2}, \quad \tilde{\gamma}_{k 3}=-\alpha \tilde{\gamma}_{k 1}, \quad \tilde{\gamma}_{k 4}=-\alpha \tilde{\gamma}_{k 2}
$$

for $k=1, \ldots, n$. Therefore $A, B_{1}, B_{2}, C_{1}, \ldots, C_{n}$ can be transformed simultaneously to hermitian matrices through the similarity transformation with

$$
T=\left[\begin{array}{ccc}
\sqrt{\alpha} & 0 & 0 \\
0 & 1 & 0 \\
0 & 0 & 1
\end{array}\right]
$$

The proof is completed.

Let us now prove that the real-diagonalizable family of the form:

$$
\left\langle\left[\begin{array}{lll}
1 & 0 & 0 \\
0 & 0 & 0 \\
0 & 0 & 0
\end{array}\right],\left[\begin{array}{rrr}
0 & * & * \\
* & 1 & 0 \\
* & 0 & -1
\end{array}\right],\left[\begin{array}{rrr}
0 & * & * \\
* & 0 & 1 \\
* & 1 & 0
\end{array}\right],\left[\begin{array}{ccc}
0 & * & * \\
* & 0 & -i \\
* & i & 0
\end{array}\right]\right\rangle .
$$

is also equivalent to a hermitian family.

Proposition 6.4. Let a nondegenerate matrix family $\left\langle A, B_{1}, B_{2}, B_{3}\right\rangle$ be spanned by

$$
\begin{aligned}
A & =\left[\begin{array}{lll}
1 & 0 & 0 \\
0 & 0 & 0 \\
0 & 0 & 0
\end{array}\right], \\
B_{1} & =\left[\begin{array}{ccc}
0 & \beta_{11}+i \tilde{\beta}_{11} & \beta_{12}+i \tilde{\beta}_{12} \\
\beta_{13}+i \tilde{\beta}_{13} & 1 & 0 \\
\beta_{14}+i \tilde{\beta}_{14} & 0 & -1
\end{array}\right], \\
B_{2} & =\left[\begin{array}{ccc}
0 & \beta_{21}+i \tilde{\beta}_{21} & \beta_{22}+i \tilde{\beta}_{22} \\
\beta_{23}+i \tilde{\beta}_{23} & 0 & 1 \\
\beta_{24}+i \tilde{\beta}_{24} & 1 & 0
\end{array}\right], \\
B_{3} & =\left[\begin{array}{ccc}
0 & \beta_{31}+i \tilde{\beta}_{31} & \beta_{32}+i \tilde{\beta}_{32} \\
\beta_{33}+i \tilde{\beta}_{33} & 0 & -i \\
\beta_{34}+i \tilde{\beta}_{34} & i & 0
\end{array}\right],
\end{aligned}
$$


where $\beta_{j k}, \tilde{\beta}_{j k}(j=1,2,3, k=1,2,3,4)$ are arbitrary real constants. Suppose that $\left\langle A, B_{1}, B_{2}, B_{3}\right\rangle$ is real-diagonalizable. Then $\left\langle A, B_{1}, B_{2}, B_{3}\right\rangle$ is equivalent to a hermitian family.

Proof. Consider

$$
\begin{aligned}
\left(\eta_{1}{ }^{2}\right. & \left.+\eta_{2}{ }^{2}-\eta_{3}{ }^{2}\right) B_{1}+2 \eta_{1} \eta_{3} B_{2}+2 \eta_{2} \eta_{3} B_{3} \\
& \equiv\left[\begin{array}{ccc}
0 & \rho_{1}(\eta)+i \tilde{\rho}_{1}(\eta) & \rho_{2}(\eta)+i \tilde{\rho}_{2}(\eta) \\
\rho_{3}(\eta)+i \tilde{\rho}_{3}(\eta) & \eta_{1}{ }^{2}+\eta_{2}{ }^{2}-\eta_{3}{ }^{2} & 2 \eta_{3}\left(\eta_{1}-i \eta_{2}\right) \\
\rho_{4}(\eta)+i \tilde{\rho}_{4}(\eta) & 2 \eta_{3}\left(\eta_{1}+i \eta_{2}\right) & -\eta_{1}{ }^{2}-\eta_{2}{ }^{2}+\eta_{3}{ }^{2}
\end{array}\right]
\end{aligned}
$$

with any fixed $\eta=\left(\eta_{1}, \eta_{2}, \eta_{3}\right) \in \mathbb{R}^{3} \backslash\{(0,0,0)\}$. Here

$$
\begin{aligned}
& \rho_{j}(\eta) \equiv \beta_{1 j}\left(\eta_{1}{ }^{2}+\eta_{2}{ }^{2}-\eta_{3}{ }^{2}\right)+2 \beta_{2 j} \eta_{1} \eta_{3}+2 \beta_{3 j} \eta_{2} \eta_{3}, \\
& \tilde{\rho}_{j}(\eta) \equiv \tilde{\beta}_{1 j}\left(\eta_{1}{ }^{2}+\eta_{2}{ }^{2}-\eta_{3}{ }^{2}\right)+2 \tilde{\beta}_{2 j} \eta_{1} \eta_{3}+2 \tilde{\beta}_{3 j} \eta_{2} \eta_{3} .
\end{aligned}
$$

for $j=1,2,3,4$. Note that

$$
\left\langle A,\left(\eta_{1}{ }^{2}+\eta_{2}{ }^{2}-\eta_{3}{ }^{2}\right) B_{1}+2 \eta_{1} \eta_{3} B_{2}+2 \eta_{2} \eta_{3} B_{3}\right\rangle \subset\left\langle A, B_{1}, B_{2}, B_{3}\right\rangle
$$

is clearly real-diagonalizable.

We define a unitary matrix $U(\eta)$ by

$$
U(\eta) \equiv\left[\begin{array}{ccc}
1 & 0 & 0 \\
0 & \left(\eta_{1}-i \eta_{2}\right) /\|\eta\| & -\eta_{3} /\|\eta\| \\
0 & \eta_{3} /\|\eta\| & \left(\eta_{1}+i \eta_{2}\right) /\|\eta\|
\end{array}\right]
$$

where

$$
\|\eta\|=\left(\eta_{1}{ }^{2}+\eta_{2}{ }^{2}+\eta_{3}{ }^{2}\right)^{1 / 2}
$$

By the similarity transformation with this $U(\eta)$,

$$
\left\langle A,\left(\eta_{1}{ }^{2}+\eta_{2}{ }^{2}-\eta_{3}{ }^{2}\right) B_{1}+2 \eta_{1} \eta_{3} B_{2}+2 \eta_{2} \eta_{3} B_{3}\right\rangle
$$

is equivalent to

$$
\left\langle\left[\begin{array}{lll}
1 & 0 & 0 \\
0 & 0 & 0 \\
0 & 0 & 0
\end{array}\right],\|\eta\|^{-1} \cdot\left[\begin{array}{ccc}
0 & \sigma_{1}(\eta)+i \tilde{\sigma}_{1}(\eta) & \sigma_{2}(\eta)+i \tilde{\sigma}_{2}(\eta) \\
\sigma_{3}(\eta)+i \tilde{\sigma}_{3}(\eta) & \|\eta\|^{3} & 0 \\
\sigma_{4}(\eta)+i \tilde{\sigma}_{4}(\eta) & 0 & -\|\eta\|^{3}
\end{array}\right]\right\rangle
$$

where

$$
\begin{aligned}
\sigma_{1}(\eta) \equiv & \left(\eta_{1}^{2}+\eta_{2}{ }^{2}-\eta_{3}{ }^{2}\right)\left(\beta_{11} \eta_{1}+\tilde{\beta}_{11} \eta_{2}+\beta_{12} \eta_{3}\right) \\
& +2 \eta_{1} \eta_{3}\left(\beta_{21} \eta_{1}+\tilde{\beta}_{21} \eta_{2}+\beta_{22} \eta_{3}\right) \\
& +2 \eta_{2} \eta_{3}\left(\beta_{31} \eta_{1}+\tilde{\beta}_{31} \eta_{2}+\beta_{32} \eta_{3}\right)
\end{aligned}
$$




$$
\begin{aligned}
& \tilde{\sigma}_{1}(\eta) \equiv\left(\eta_{1}{ }^{2}+\eta_{2}{ }^{2}-\eta_{3}{ }^{2}\right)\left(\tilde{\beta}_{11} \eta_{1}-\beta_{11} \eta_{2}+\tilde{\beta}_{12} \eta_{3}\right) \\
& +2 \eta_{1} \eta_{3}\left(\tilde{\beta}_{21} \eta_{1}-\beta_{21} \eta_{2}+\tilde{\beta}_{22} \eta_{3}\right) \\
& +2 \eta_{2} \eta_{3}\left(\tilde{\beta}_{31} \eta_{1}-\beta_{31} \eta_{2}+\tilde{\beta}_{32} \eta_{3}\right) \\
& \sigma_{2}(\eta) \equiv\left(\eta_{1}{ }^{2}+\eta_{2}{ }^{2}-\eta_{3}{ }^{2}\right)\left(\beta_{12} \eta_{1}-\tilde{\beta}_{12} \eta_{2}-\beta_{11} \eta_{3}\right) \\
& +2 \eta_{1} \eta_{3}\left(\beta_{22} \eta_{1}-\tilde{\beta}_{22} \eta_{2}-\beta_{21} \eta_{3}\right) \\
& +2 \eta_{2} \eta_{3}\left(\beta_{32} \eta_{1}-\tilde{\beta}_{32} \eta_{2}-\beta_{31} \eta_{3}\right) \\
& \tilde{\sigma}_{2}(\eta) \equiv\left(\eta_{1}{ }^{2}+\eta_{2}{ }^{2}-\eta_{3}{ }^{2}\right)\left(\tilde{\beta}_{12} \eta_{1}+\beta_{12} \eta_{2}-\tilde{\beta}_{11} \eta_{3}\right) \\
& +2 \eta_{1} \eta_{3}\left(\tilde{\beta}_{22} \eta_{1}+\beta_{22} \eta_{2}-\tilde{\beta}_{21} \eta_{3}\right) \\
& +2 \eta_{2} \eta_{3}\left(\tilde{\beta}_{32} \eta_{1}+\beta_{32} \eta_{2}-\tilde{\beta}_{31} \eta_{3}\right) \\
& \sigma_{3}(\eta) \equiv\left(\eta_{1}{ }^{2}+\eta_{2}{ }^{2}-\eta_{3}{ }^{2}\right)\left(\beta_{13} \eta_{1}-\tilde{\beta}_{13} \eta_{2}+\beta_{14} \eta_{3}\right) \\
& +2 \eta_{1} \eta_{3}\left(\beta_{23} \eta_{1}-\tilde{\beta}_{23} \eta_{2}+\beta_{24} \eta_{3}\right) \\
& +2 \eta_{2} \eta_{3}\left(\beta_{33} \eta_{1}-\tilde{\beta}_{33} \eta_{2}+\beta_{34} \eta_{3}\right) \text {, } \\
& \tilde{\sigma}_{3}(\eta) \equiv\left(\eta_{1}{ }^{2}+\eta_{2}{ }^{2}-\eta_{3}{ }^{2}\right)\left(\widetilde{\beta}_{13} \eta_{1}+\beta_{13} \eta_{2}+\tilde{\beta}_{14} \eta_{3}\right) \\
& +2 \eta_{1} \eta_{3}\left(\tilde{\beta}_{23} \eta_{1}+\beta_{23} \eta_{2}+\tilde{\beta}_{24} \eta_{3}\right) \\
& +2 \eta_{2} \eta_{3}\left(\tilde{\beta}_{33} \eta_{1}+\beta_{33} \eta_{2}+\tilde{\beta}_{34} \eta_{3}\right) \text {, } \\
& \sigma_{4}(\eta) \equiv\left(\eta_{1}{ }^{2}+\eta_{2}{ }^{2}-\eta_{3}{ }^{2}\right)\left(\beta_{14} \eta_{1}+\tilde{\beta}_{14} \eta_{2}-\beta_{13} \eta_{3}\right) \\
& +2 \eta_{1} \eta_{3}\left(\beta_{24} \eta_{1}+\tilde{\beta}_{24} \eta_{2}-\beta_{23} \eta_{3}\right) \\
& +2 \eta_{2} \eta_{3}\left(\beta_{34} \eta_{1}+\tilde{\beta}_{34} \eta_{2}-\beta_{33} \eta_{3}\right) \text {, } \\
& \tilde{\sigma}_{4}(\eta) \equiv\left(\eta_{1}{ }^{2}+\eta_{2}{ }^{2}-\eta_{3}{ }^{2}\right)\left(\tilde{\beta}_{14} \eta_{1}-\beta_{14} \eta_{2}-\tilde{\beta}_{13} \eta_{3}\right) \\
& +2 \eta_{1} \eta_{3}\left(\tilde{\beta}_{24} \eta_{1}-\beta_{24} \eta_{2}-\tilde{\beta}_{23} \eta_{3}\right) \\
& +2 \eta_{2} \eta_{3}\left(\tilde{\beta}_{34} \eta_{1}-\beta_{34} \eta_{2}-\tilde{\beta}_{33} \eta_{3}\right) \text {. }
\end{aligned}
$$

From Proposition 4.3, either

$$
\left\{\sigma_{2}(\eta)+i \tilde{\sigma}_{2}(\eta)\right\}\left\{\sigma_{4}(\eta)+i \tilde{\sigma}_{4}(\eta)\right\}>0
$$

or

$$
\sigma_{2}(\eta)+i \tilde{\sigma}_{2}(\eta)=\sigma_{4}(\eta)+i \tilde{\sigma}_{4}(\eta)=0
$$

holds for any $\eta \in \mathbb{R}^{3}$. By applying Lemma 3.2, we have

$$
\begin{array}{lll}
\sigma_{2}(\eta) \equiv \mu(\eta) \varphi(\eta), & & \tilde{\sigma}_{2}(\eta) \equiv \tilde{\mu}(\eta) \varphi(\eta), \\
\sigma_{4}(\eta) \equiv \mu(\eta) \psi(\eta), & & \tilde{\sigma}_{4}(\eta) \equiv-\tilde{\mu}(\eta) \psi(\eta)
\end{array}
$$


where $\mu(\eta), \tilde{\mu}(\eta), \varphi(\eta), \psi(\eta)$ are polynomials with real coefficients such that

$$
\operatorname{sgn} \varphi(\eta)=\operatorname{sgn} \psi(\eta)
$$

for all $\eta \in \mathbb{R}^{3}$ unless $\mu(\eta)=\tilde{\mu}(\eta)=0$. Note that $\varphi(\eta)$ and $\psi(\eta)$ are polynomials of the same degree, equal to or less than three (see (6.33) and (6.34) and remark that $\sigma_{2}(\eta)$ and $\sigma_{4}(\eta)$ are both cubic). Similarly, we have also

$$
\begin{aligned}
& \sigma_{1}(\eta) \equiv \mu_{0}(\eta) \varphi_{0}(\eta), \quad \tilde{\sigma}_{1}(\eta) \equiv \tilde{\mu}_{0}(\eta) \varphi_{0}(\eta), \\
& \sigma_{3}(\eta) \equiv \mu_{0}(\eta) \psi_{0}(\eta), \quad \tilde{\sigma}_{3} \equiv-\tilde{\mu}_{0}(\eta) \psi_{0}(\eta)
\end{aligned}
$$

where $\mu_{0}(\eta), \tilde{\mu}_{0}(\eta), \varphi_{0}(\eta), \psi_{0}(\eta)$ are polynomials with real coefficients such that

$$
\operatorname{sgn} \varphi_{0}(\eta)=\operatorname{sgn} \psi_{0}(\eta)
$$

for all $\eta \in \mathbb{R}^{3}$ unless $\mu_{0}(\eta)=\tilde{\mu}_{0}(\eta)=0$.

Before proceeding further, let us prove if each of $\varphi(\eta)$ and $\psi(\eta)$ is a positive constant multiple of the other, then $\left\langle A, B_{1}, B_{2}, B_{3}\right\rangle$ is equivalent to a hermitian family. Putting

$$
\psi(\eta) \equiv \alpha \varphi(\eta)
$$

with a real constant $\alpha>0$, we obtain

$$
\sigma_{4}\left(\eta_{1}, \eta_{2}, \eta_{3}\right) \equiv \alpha \sigma_{2}\left(\eta_{1}, \eta_{2}, \eta_{3}\right) .
$$

From the definition of $\sigma_{2}(\eta)$ and $\sigma_{4}(\eta)$, this means

$$
\begin{array}{ll}
\beta_{j 3}=\alpha \beta_{j 1}, & \beta_{j 4}=\alpha \beta_{j 2}, \\
\tilde{\beta}_{j 3}=-\alpha \tilde{\beta}_{j 1}, & \tilde{\beta}_{j 4}=-\alpha \tilde{\beta}_{j 2},
\end{array}
$$

for $j=1,2,3$. Through the similarity transformation with

$$
T=\left[\begin{array}{ccc}
\sqrt{\alpha} & 0 & 0 \\
0 & 1 & 0 \\
0 & 0 & 1
\end{array}\right],
$$

$\left\langle A, B_{1}, B_{2}, B_{3}\right\rangle$ is equivalent to a hermitian family. Similarly, we can prove if each of $\varphi_{0}(\eta)$ and $\psi_{0}(\eta)$ is a positive multiple of the other then $\left\langle A, B_{1}, B_{2}, B_{3}\right\rangle$ is equivalent to a hermitian family.

Let us now prove that $\left\langle A, B_{1}, B_{2}, B_{3}\right\rangle$ is equivalent to a hermitian family by contradiction. Assume the contrary. By the above-mentioned remark, we may further assume that $\varphi(\eta)$ is not a positive constant multiple of $\psi(\eta)$, nor is $\varphi_{0}(\eta)$ a positive constant multiple of $\psi_{0}(\eta)$.

First let us prove

$$
\sigma_{2}(\eta)+i \tilde{\sigma}_{2}(\eta)=\sigma_{4}(\eta)+i \tilde{\sigma}_{4}(\eta)=0
$$


for some $\eta \in \mathbb{R}^{3} \backslash\{(0,0,0)\}$, by contradiction. Assume the contrary. We may also assume that $\mu(\eta)$ and $\tilde{\mu}(\eta)$ do not simultaneously vanish because

$$
\mu(\eta)=\tilde{\mu}(\eta)=0
$$

would mean

$$
\sigma_{2}(\eta)+i \tilde{\sigma}_{2}(\eta)=\sigma_{4}(\eta)+i \tilde{\sigma}_{4}(\eta)=0
$$

from their definition. Thus the equality

$$
\operatorname{sgn} \varphi(\eta) \equiv \operatorname{sgn} \psi(\eta)
$$

mentioned in Lemma 3.2 is valid also for $\eta$ with $\mu(\eta)=\tilde{\mu}(\eta)=0$, hence for all $\eta \in \mathbb{R}^{3}$. Moreover, $\mu(\eta)$ and $\tilde{\mu}(\eta)$ must have even degree because otherwise they would have a common nontrivial real zero point. Because $\varphi(\eta)$ and $\psi(\eta)$ are linear or cubic (recall $\mu(\eta)$ and $\tilde{\mu}(\eta)$ are of even degree), they have nontrivial zero points which must be common from (6.35). In order to prove this fact rigorously, we need only regard $\varphi(\eta)=0$ and $\psi(\eta)=0$ as two algebraic curves in $\mathbb{R P}^{2}$ and apply the Bézout theorem (see, for example, Brieskorn-Knörrer [1]). Note that the curves $\varphi(\eta)=0$ and $\psi(\eta)=0$ have an odd number of common (complex) points in $\mathbb{C P}^{2}$. Note also that common complex zero points of $\varphi(\eta)$ and $\psi(\eta)$ appear with their conjugates. Therefore, there must be at least one real nontrivial common zero point. Thus we have proved

$$
\sigma_{2}(\eta)+i \tilde{\sigma}_{2}(\eta)=\sigma_{4}(\eta)+i \tilde{\sigma}_{4}(\eta)=0
$$

for some $\eta \in \mathbb{R}^{3} \backslash\{(0,0,0)\}$.

The fact just proved means that through the similarity transformation with unitary matrix

$$
U(\eta) \equiv\left[\begin{array}{ccc}
1 & 0 & 0 \\
0 & \left(\eta_{1}-i \eta_{2}\right) /\|\eta\| & -\eta_{3} /\|\eta\| \\
0 & \eta_{3} /\|\eta\| & \left(\eta_{1}+i \eta_{2}\right) /\|\eta\|
\end{array}\right]
$$

with $\eta=\left(\eta_{1}, \eta_{2}, \eta_{3}\right)$ found above,

$$
\left(\eta_{1}^{2}+\eta_{2}^{2}-\eta_{3}^{2}\right) B_{1}+2 \eta_{1} \eta_{3} B_{2}+2 \eta_{2} \eta_{3} B_{3}
$$

becomes

$$
\|\eta\|^{-1} \cdot\left[\begin{array}{ccc}
0 & \sigma_{1}(\eta)+i \tilde{\sigma}_{1}(\eta) & \sigma_{2}(\eta)+i \tilde{\sigma}_{2}(\eta) \\
\sigma_{3}(\eta)+i \tilde{\sigma}_{3}(\eta) & \|\eta\|^{3} & 0 \\
\sigma_{4}(\eta)+i \tilde{\sigma}_{4}(\eta) & 0 & -\|\eta\|^{3}
\end{array}\right]
$$

where

$$
\sigma_{2}(\eta)+i \tilde{\sigma}_{2}(\eta)=\sigma_{4}(\eta)+i \tilde{\sigma}_{4}(\eta)=0
$$


By using this similarity transformation, we may further assume that

$$
\beta_{12}+i \tilde{\beta}_{12}=\beta_{14}+i \tilde{\beta}_{14}=0
$$

holds for $B_{1}$ of $\left\langle A, B_{1}, B_{2}, B_{3}\right\rangle$. And by another similarity transformation with

$$
T=\left[\begin{array}{ccc}
c & 0 & 0 \\
0 & 1 & 0 \\
0 & 0 & 1
\end{array}\right] \quad(c \neq 0: \text { complex })
$$

the $(1,2)$ - and $(2,1)$ - entries of $B_{1}$, namely, $\beta_{11}+i \tilde{\beta}_{11}$ and $\beta_{13}+i \tilde{\beta}_{13}$ become real (see Proposition 4.3). In other words, we have $\tilde{\beta}_{11}=\tilde{\beta}_{13}=0$. Summing up, we may even assume

$$
\tilde{\beta}_{11}=\tilde{\beta}_{13}=\beta_{12}=\tilde{\beta}_{12}=\beta_{14}=\tilde{\beta}_{14}=0,
$$

without loss of generality. Therefore, $\sigma_{2}(\eta), \tilde{\sigma}_{2}(\eta), \sigma_{4}(\eta), \tilde{\sigma}_{4}(\eta)$ are reduced to

$$
\begin{aligned}
\sigma_{2}(\eta) \equiv & \eta_{3}\left\{-\beta_{11}\left(\eta_{1}^{2}+\eta_{2}^{2}-\eta_{3}^{2}\right)+2 \eta_{1}\left(\beta_{22} \eta_{1}-\tilde{\beta}_{22} \eta_{2}-\beta_{21} \eta_{3}\right)\right. \\
& \left.+2 \eta_{2}\left(\beta_{32} \eta_{1}-\tilde{\beta}_{32} \eta_{2}-\beta_{31} \eta_{3}\right)\right\} \\
\tilde{\sigma}_{2}(\eta) \equiv & 2 \eta_{3}\left\{\eta_{1}\left(\tilde{\beta}_{22} \eta_{1}+\beta_{22} \eta_{2}-\tilde{\beta}_{21} \eta_{3}\right)+\eta_{2}\left(\tilde{\beta}_{32} \eta_{1}+\beta_{32} \eta_{2}-\tilde{\beta}_{31} \eta_{3}\right)\right\} \\
\sigma_{4}(\eta) \equiv & \eta_{3}\left\{-\beta_{13}\left(\eta_{1}^{2}+\eta_{2}^{2}-\eta_{3}^{2}\right)+2 \eta_{1}\left(\beta_{24} \eta_{1}+\tilde{\beta}_{24} \eta_{2}-\beta_{23} \eta_{3}\right)\right. \\
& \left.+2 \eta_{2}\left(\beta_{34} \eta_{1}+\tilde{\beta}_{34} \eta_{2}-\beta_{33} \eta_{3}\right)\right\} \\
\tilde{\sigma}_{4}(\eta) \equiv & 2 \eta_{3}\left\{\eta_{1}\left(\tilde{\beta}_{24} \eta_{1}-\beta_{24} \eta_{2}-\tilde{\beta}_{23} \eta_{3}\right)+\eta_{2}\left(\tilde{\beta}_{34} \eta_{1}-\beta_{34} \eta_{2}-\tilde{\beta}_{33} \eta_{3}\right)\right\}
\end{aligned}
$$

By applying Lemma 3.2 again, we have

$$
\begin{array}{lll}
\sigma_{2}(\eta) \equiv \mu(\eta) \varphi(\eta), & & \tilde{\sigma}_{2}(\eta) \equiv \tilde{\mu}(\eta) \varphi(\eta), \\
\sigma_{4}(\eta) \equiv \mu(\eta) \psi(\eta), & & \tilde{\sigma}_{4}(\eta) \equiv-\tilde{\mu}(\eta) \psi(\eta) .
\end{array}
$$

Here $\varphi(\eta), \psi(\eta)$ must have a common factor $\eta_{3}$. Let us divide the case according to the degree of $\varphi(\eta)$ and $\psi(\eta)$. Assume first that they are linear. In this case, they are both constant multiples of $\eta_{3}$. And the equality

$$
\operatorname{sgn} \varphi(\eta)=\operatorname{sgn} \psi(\eta) \quad \text { unless } \quad \mu(\eta)=\tilde{\mu}(\eta)=0
$$

means that each of $\varphi(\eta)$ and $\psi(\eta)$ is a positive multiple of the other, which contradicts our assumption. Similarly, we reach a contradiction also in the case where $\varphi(\eta)$ and $\psi(\eta)$ are quadratic polynomials which have $\eta_{3}$ as a factor. Finally we assume that $\varphi(\eta)$ and $\psi(\eta)$ are cubic polynomials which have $\eta_{3}$ as a factor. Then $\mu(\eta)$ and $\tilde{\mu}(\eta)$ become nonzero constants. Therefore, $\tilde{\sigma}_{2}(\eta) / \sigma_{2}(\eta)$ and $\tilde{\sigma}_{4}(\eta) / \sigma_{4}(\eta)$ are nonzero constants. Hence (6.41) and (6.42) mean $\beta_{11}=0$, considering the coefficients of $\eta_{3}{ }^{3}$. Similarly (6.43) and (6.44) mean $\beta_{13}=0$. Thus we have 


$$
\beta_{11}=\beta_{13}=0 .
$$

Now if $\sigma_{2}(\eta) / \eta_{3}, \tilde{\sigma}_{2}(\eta) / \eta_{3}, \sigma_{4}(\eta) / \eta_{3}, \tilde{\sigma}_{4}(\eta) / \eta_{3}$ were linear polynomials with respect to $\eta_{3}$ then each of $\varphi(\eta)$ and $\psi(\eta)$ would be a positive constant multiple of the other, because of $\operatorname{sgn} \varphi(\eta)=\operatorname{sgn} \psi(\eta)$. This means that $\sigma_{2}(\eta) / \eta_{3}, \tilde{\sigma}_{2}(\eta) / \eta_{3}$, $\sigma_{4}(\eta) / \eta_{3}, \tilde{\sigma}_{4}(\eta) / \eta_{3}$ are constants with respect to $\eta_{3}$, that is,

$$
\beta_{21}=\tilde{\beta}_{21}=\beta_{31}=\tilde{\beta}_{31}=\beta_{23}=\tilde{\beta}_{23}=\beta_{33}=\tilde{\beta}_{33}=0 .
$$

Now let us consider $\sigma_{1}(\eta)+i \tilde{\sigma}_{1}(\eta), \sigma_{3}(\eta)+i \tilde{\sigma}(\eta)$ :

$$
\begin{aligned}
& \sigma_{1}(\eta) \equiv 2 \eta_{3}{ }^{2}\left(\beta_{22} \eta_{1}+\beta_{32} \eta_{2}\right), \\
& \tilde{\sigma}_{1}(\eta) \equiv 2 \eta_{3}{ }^{2}\left(\tilde{\beta}_{22} \eta_{1}+\tilde{\beta}_{32} \eta_{2}\right), \\
& \sigma_{3}(\eta) \equiv 2 \eta_{3}{ }^{2}\left(\beta_{24} \eta_{1}+\beta_{34} \eta_{2}\right), \\
& \tilde{\sigma}_{3}(\eta) \equiv 2 \eta_{3}{ }^{2}\left(\tilde{\beta}_{24} \eta_{1}+\tilde{\beta}_{34} \eta_{2}\right) .
\end{aligned}
$$

Here we have used (6.40), (6.45), (6.46). Again from Lemma 3.2, there must be a positive constant $\alpha>0$ such that

$$
\begin{aligned}
& \sigma_{3}(\eta) \equiv \alpha \sigma_{1}(\eta), \\
& \tilde{\sigma}_{3}(\eta) \equiv-\alpha \tilde{\sigma}_{1}(\eta) .
\end{aligned}
$$

This would mean that $\left\langle A, B_{1}, B_{2}, B_{3}\right\rangle$ is equivalent to a hermitian family as pointed out before. We are thus led to a contradiction.

Before ending this section, we shall consider non-uniformly realdiagonalizable families. In other words, we shall consider

$$
\left\langle\left[\begin{array}{lll}
1 & 0 & 0 \\
0 & 0 & 0 \\
0 & 0 & 0
\end{array}\right],\left[\begin{array}{lll}
0 & 1 & 0 \\
1 & 0 & 1 \\
0 & 0 & 0
\end{array}\right],\left[\begin{array}{lll}
0 & * & * \\
* & 0 & * \\
* & 0 & 0
\end{array}\right],\left[\begin{array}{lll}
0 & * & * \\
* & 0 & * \\
* & 0 & 0
\end{array}\right]\right\rangle
$$

or

$$
\left\langle\left[\begin{array}{lll}
1 & 0 & 0 \\
0 & 0 & 0 \\
0 & 0 & 0
\end{array}\right],\left[\begin{array}{lll}
0 & 1 & 0 \\
1 & 0 & 1 \\
0 & 0 & 0
\end{array}\right],\left[\begin{array}{rrr}
0 & * & * \\
* & 1 & * \\
* & 0 & -1
\end{array}\right],\left[\begin{array}{lll}
0 & * & * \\
* & 0 & * \\
* & 0 & 0
\end{array}\right]\right\rangle
$$

where each $*$ stands for a complex constant.

Proposition 6.5. Let a matrix family $\left\langle A, B, C_{1}, \ldots, C_{n}\right\rangle(n \geq 2)$ be spanned by

$$
A=\left[\begin{array}{lll}
1 & 0 & 0 \\
0 & 0 & 0 \\
0 & 0 & 0
\end{array}\right], \quad B=\left[\begin{array}{lll}
0 & 1 & 0 \\
1 & 0 & 1 \\
0 & 0 & 0
\end{array}\right]
$$




$$
C_{j}=\left[\begin{array}{ccc}
0 & \gamma_{j 1}+i \tilde{\gamma}_{j 1} & \gamma_{j 2}+i \tilde{\gamma}_{j 2} \\
\gamma_{j 3}+i \tilde{\gamma}_{j 3} & 0 & \gamma_{j 5}+i \tilde{\gamma}_{j 5} \\
\gamma_{j 4}+i \tilde{\gamma}_{j 4} & 0 & 0
\end{array}\right]
$$

where $\gamma_{j k}, \tilde{\gamma}_{j k}(j=1, \ldots, n ; k=1, \ldots, 5)$ are real constants. If $\left\langle A, B, C_{1}, \ldots, C_{n}\right\rangle$ is real-diagonalizable, then it is degenerate.

Proof. Assume, to the contrary, that $\left\langle A, B, C_{1}, \ldots, C_{n}\right\rangle$ is nondegenerate. Then by a suitable change of basis, we may further assume

$$
\gamma_{11}=\tilde{\gamma}_{11}=0
$$

Hence we are led to a contradiction, by applying Lemma 5.12 to $\left\langle A, B, C_{1}\right\rangle$.

Proposition 6.6. Let a nondegenerate matrix family $\langle A, B, C\rangle$ be spanned by

$$
\begin{array}{ll}
A & =\left[\begin{array}{lll}
1 & 0 & 0 \\
0 & 0 & 0 \\
0 & 0 & 0
\end{array}\right], \quad B=\left[\begin{array}{lll}
0 & 1 & 0 \\
1 & 0 & 1 \\
0 & 0 & 0
\end{array}\right], \\
C & =\left[\begin{array}{ccc}
0 & \gamma_{1}+i \tilde{\gamma}_{1} & \gamma_{2}+i \tilde{\gamma}_{2} \\
\gamma_{3}+i \tilde{\gamma}_{3} & 1 & \gamma_{5}+i \tilde{\gamma}_{5} \\
\gamma_{4}+i \tilde{\gamma}_{4} & 0 & -1
\end{array}\right], \quad D=\left[\begin{array}{ccc}
0 & \delta_{1}+i \tilde{\delta}_{1} & \delta_{2}+i \tilde{\delta}_{2} \\
\delta_{3}+i \tilde{\delta}_{3} & 0 & \delta_{5}+i \tilde{\delta}_{5} \\
\delta_{4}+i \tilde{\delta}_{4} & 0 & 0
\end{array}\right]
\end{array}
$$

where $\gamma_{j}, \tilde{\gamma}_{j}, \delta_{j}, \tilde{\delta}_{j}(j=1, \ldots, 5)$ are real constants. Then $\langle A, B, C, D\rangle$ is (nonuniformly) real-diagonalizable if and only if it is equivalent to

$$
\left\langle\left[\begin{array}{lll}
1 & 0 & 0 \\
0 & 0 & 0 \\
0 & 0 & 0
\end{array}\right],\left[\begin{array}{lll}
0 & 1 & 0 \\
1 & 0 & 1 \\
0 & 0 & 0
\end{array}\right],\left[\begin{array}{ccc}
0 & \beta & -\gamma \\
\beta(1-\alpha) & 1 & 0 \\
-2 \alpha & 0 & -1
\end{array}\right],\left[\begin{array}{ccc}
0 & -i & \delta+\frac{i}{2} \beta \\
i & 0 & i \\
0 & 0 & 0
\end{array}\right]\right\rangle
$$

where the real constants satisfy

$$
0<\alpha<1, \quad \gamma>\frac{1}{8} \beta^{2}+\frac{1}{2} \delta^{2} .
$$

Remark. By using a further change of basis and similarity transformation, we can assume

$$
\delta=0
$$

in the above matrix family.

Proof. Let us prove the only-if part. First note that, replacing $C$ by $C-\gamma_{5} B, D$ by $D-\delta_{5} B$, we may assume

$$
\gamma_{5}=0, \quad \delta_{5}=0
$$


Let us apply Lemma 5.14 to $\langle A, B, C\rangle$ and $\langle A, B, C+D\rangle$. Thus we have especially

$$
\tilde{\gamma}_{3}=-\tilde{\gamma}_{1}, \quad \tilde{\gamma}_{4}=0, \quad \tilde{\gamma}_{5}=-\tilde{\gamma}_{1},
$$

and

$$
\tilde{\gamma}_{3}+\tilde{\delta}_{3}=-\tilde{\gamma}_{1}-\tilde{\delta}_{1}, \quad \tilde{\gamma}_{5}+\tilde{\delta}_{5}=-\tilde{\gamma}_{1}-\tilde{\delta}_{1} .
$$

Combining these, we have also

$$
\tilde{\delta}_{3}=-\tilde{\delta}_{1}, \quad \tilde{\delta}_{5}=-\tilde{\delta}_{1} .
$$

Then we apply Lemma 5.12 to $\left\langle A, B, D-\delta_{1} B\right\rangle$, we have

$$
\tilde{\delta}_{1} \neq 0, \quad \delta_{1}=\delta_{3}, \quad \delta_{4}=\tilde{\delta}_{4}=0 .
$$

Therefore, replacing $C$ by $C-\left(\tilde{\gamma}_{1} / \tilde{\delta}_{1}\right) D, D$ by $-\left(1 / \tilde{\delta}_{1}\right) D$, we may assume

$$
\gamma_{5}=\tilde{\gamma}_{1}=\tilde{\gamma}_{3}=\tilde{\gamma}_{4}=\tilde{\gamma}_{5}=0 \text {, }
$$

$$
\tilde{\delta}_{1}=-1, \quad \tilde{\delta}_{3}=\tilde{\delta}_{5}=1, \quad \delta_{1}=\delta_{3}, \quad \delta_{4}=\tilde{\delta}_{4}=\delta_{5}=0
$$

Now applying Lemma 5.14 to $\langle A, B, C\rangle$, we obtain

$$
\tilde{\gamma}_{2}=\gamma_{1} \tilde{\gamma}_{1}=0, \quad \gamma_{3}=\frac{1}{2} \gamma_{1}\left(\gamma_{4}+2\right), \quad-2<\gamma_{4}<0, \quad \gamma_{2}<-\frac{1}{8} \gamma_{1}^{2}
$$

Next let us apply the same Lemma 5.14 to $\langle A, B, C+\eta D\rangle$ with arbitrarily fixed $\eta \in \mathbb{R}$. So we must have

$$
\left(\tilde{\gamma}_{2}+\tilde{\delta}_{2} \eta\right) \equiv-\frac{1}{2}\left(\gamma_{1}+\delta_{1} \eta\right)\left(\tilde{\gamma}_{1}+\tilde{\delta}_{1} \eta\right)
$$

namely

$$
\tilde{\delta}_{2} \eta \equiv \frac{\eta}{2}\left(\gamma_{1}+\delta_{1} \eta\right)
$$

holds for all $\eta \in \mathbb{R}$. Thus we obtain

$$
\delta_{1}\left(=\delta_{3}\right)=0, \quad \tilde{\delta}_{2}=\frac{1}{2} \gamma_{1} .
$$

Now, it suffices to consider $\langle A, B, C+\eta D\rangle$ with arbitrarily fixed $\eta \in \mathbb{R}$ because $\langle A, B, D\rangle$ is real-diagonalizable as easily seen. So let us apply Lemma 5.14. Thus the required condition is that the following hold as well as (6.49), (6.50), (6.51), (6.52).

$$
\gamma_{2}+\delta_{2} \eta<\frac{1}{8}\left(4 \eta^{2}-\gamma_{1}^{2}\right) \quad \text { for all } \quad \eta \in \mathbb{R} .
$$

As easily seen, the last inequality holds if and only if

$$
\gamma_{2}<-\frac{1}{2} \delta_{2}^{2}-\frac{1}{8} \gamma_{1}^{2}
$$


Finally, introducing new real parameters $\alpha, \beta, \gamma, \delta$ by

$$
\gamma_{1}=\beta, \quad \gamma_{2}=-\gamma, \quad \gamma_{4}=-2 \alpha, \quad \delta_{2}=\delta,
$$

we complete the proof of the only-if part. The if part is clear by the last argument.

Let us summarize the results obtained in this section as a theorem.

Theorem 6.7. Let $\left\langle A_{1}, A_{2}, A_{3}, A_{4}\right\rangle$ be a nondegenerate $3 \times 3$ matrix family. Then the following 1) and 2) hold.

1) Suppose that the family $\left\langle A_{1}, A_{2}, A_{3}, A_{4}\right\rangle$ has multiple eigenvalues and is not equivalent to any hermitian family. Then it is uniformly real-diagonalizable if and only if it is equivalent to either

$\left\langle\left[\begin{array}{lll}1 & 0 & 0 \\ 0 & 0 & 0 \\ 0 & 0 & 0\end{array}\right],\left[\begin{array}{lll}0 & 1 & 0 \\ 1 & 0 & 0 \\ 0 & 0 & 0\end{array}\right],\left[\begin{array}{lll}0 & 0 & 1 \\ 0 & 0 & 0 \\ 1 & 0 & 0\end{array}\right],\left[\begin{array}{ccc}0 & -c+\frac{1}{c} & -i\left(c+\frac{1}{c}\right) \\ c-\frac{1}{c} & 0 & 0 \\ i\left(c+\frac{1}{c}\right) & 0 & 0\end{array}\right]\right\rangle$

with $c$ a complex constant satisfying $|c| \neq 0,1$, or

$\left\langle\left[\begin{array}{lll}1 & 0 & 0 \\ 0 & 0 & 0 \\ 0 & 0 & 0\end{array}\right],\left[\begin{array}{lll}0 & 1 & 0 \\ 1 & 0 & 0 \\ 0 & 0 & 0\end{array}\right],\left[\begin{array}{ccc}0 & -\alpha & 1 \\ \alpha & 0 & 0 \\ 1 & 0 & 0\end{array}\right],\left[\begin{array}{ccc}0 & -i & (1+\gamma)\left(\beta+i \frac{\alpha}{\gamma}\right) \\ i & 0 & 0 \\ (1-\gamma)\left(\beta-i \frac{\alpha}{\gamma}\right) & 0 & 0\end{array}\right]\right\rangle$

where $0<\alpha<1, \beta \neq 0, \gamma \neq 0$ are real constants satisfying $\beta^{2} \gamma^{2}<1-\alpha^{2}$, or

$$
\left\langle\left[\begin{array}{lll}
1 & 0 & 0 \\
0 & 0 & 0 \\
0 & 0 & 0
\end{array}\right],\left[\begin{array}{lll}
0 & 1 & 0 \\
1 & 0 & 0 \\
0 & 0 & 0
\end{array}\right],\left[\begin{array}{ccc}
0 & -\alpha & 1 \\
\alpha & 0 & 0 \\
1 & 0 & 0
\end{array}\right],\left[\begin{array}{ccc}
0 & -i & i(\alpha+\gamma) \\
i & 0 & 0 \\
i(\alpha-\gamma) & 0 & 0
\end{array}\right]\right\rangle
$$

where $0<\alpha<1$ and $\gamma$ are real constants, or

$$
\left\langle\left[\begin{array}{lll}
1 & 0 & 0 \\
0 & 0 & 0 \\
0 & 0 & 0
\end{array}\right],\left[\begin{array}{lll}
0 & 1 & 0 \\
1 & 0 & 0 \\
0 & 0 & 0
\end{array}\right],\left[\begin{array}{ccc}
0 & -\alpha & 1 \\
\alpha & 0 & 0 \\
1 & 0 & 0
\end{array}\right],\left[\begin{array}{ccc}
0 & -i & (i \alpha+\gamma) \\
i & 0 & 0 \\
(i \alpha-\gamma) & 0 & 0
\end{array}\right]\right\rangle
$$

where $\alpha$ and $\gamma$ are real constants satisfying $0<\alpha<1$ and $\alpha^{2}+\gamma^{2}<1$.

2) The family $\left\langle A_{1}, A_{2}, A_{3}, A_{4}\right\rangle$ is non-uniformly real-diagonalizable (necessarily with multiple eigenvalues) if and only if it is equivalent to 


$$
\left\langle\left[\begin{array}{lll}
1 & 0 & 0 \\
0 & 0 & 0 \\
0 & 0 & 0
\end{array}\right],\left[\begin{array}{lll}
0 & 1 & 0 \\
1 & 0 & 1 \\
0 & 0 & 0
\end{array}\right],\left[\begin{array}{ccc}
0 & \beta & -\gamma \\
\beta(1-\alpha) & 1 & 0 \\
-2 \alpha & 0 & -1
\end{array}\right],\left[\begin{array}{ccc}
0 & -i & \delta+\frac{i}{2} \beta \\
i & 0 & i \\
0 & 0 & 0
\end{array}\right]\right\rangle
$$

where the real constants $\alpha, \beta, \gamma$ and $\delta$ satisfy

or their transposes.

$$
0<\alpha<1, \quad \gamma>\frac{1}{8} \beta^{2}+\frac{1}{2} \delta^{2},
$$

\section{§7. Families Spanned by Five or More Matrices}

Let us begin with the families of the form:

$$
\left.\left\langle\left[\begin{array}{lll}
1 & 0 & 0 \\
0 & 0 & 0 \\
0 & 0 & 0
\end{array}\right],\left[\begin{array}{lll}
0 & * & * \\
* & 0 & 0 \\
* & 0 & 0
\end{array}\right],\left[\begin{array}{lll}
0 & * & * \\
* & 0 & 0 \\
* & 0 & 0
\end{array}\right], \ldots\right\rangle\right\rangle
$$

Proposition 7.1. Let a nondegenerate matrix family $\left\langle A, \mathbb{B}_{1}, \ldots, \mathbb{B}_{4}\right\rangle$ be spanned by

$$
A=\left[\begin{array}{ccc}
1 & 0 & 0 \\
0 & 0 & 0 \\
0 & 0 & 0
\end{array}\right], \quad B_{k}=\left[\begin{array}{ccc}
0 & b_{k 1} & b_{k 2} \\
b_{k 3} & 0 & 0 \\
b_{k 4} & 0 & 0
\end{array}\right]
$$

where $b_{k j}(k, j=1,2,3,4)$ are complex constants. Then $\left\langle A, B_{1}, \ldots, \mathbb{B}_{4}\right\rangle$ is realdiagonalizable if and only if it is equivalent to

$$
\left\langle\left[\begin{array}{lll}
1 & 0 & 0 \\
0 & 0 & 0 \\
0 & 0 & 0
\end{array}\right],\left[\begin{array}{lll}
0 & 1 & 0 \\
1 & 0 & 0 \\
0 & 0 & 0
\end{array}\right],\left[\begin{array}{lll}
0 & 0 & 1 \\
0 & 0 & 0 \\
1 & 0 & 0
\end{array}\right],\left[\begin{array}{ccc}
0 & -\alpha & -i \\
\alpha & 0 & 0 \\
i & 0 & 0
\end{array}\right],\left[\begin{array}{ccc}
0 & -i & \alpha \\
i & 0 & 0 \\
-\alpha & 0 & 0
\end{array}\right]\right\rangle
$$

with a real constant $\alpha$ satisfying $0 \leq \alpha<1$. Moreover, in this case, $\left\langle A, B_{1}, \ldots, B_{4}\right\rangle$ is uniformly real-diagonalizable.

Proof. Repeating the same argument as in the proof of Proposition 6.1, we may assume

$$
B_{1}=\left[\begin{array}{lll}
0 & 1 & 0 \\
1 & 0 & 0 \\
0 & 0 & 0
\end{array}\right], \quad B_{2}=\left[\begin{array}{lll}
0 & 0 & 1 \\
0 & 0 & 0 \\
1 & 0 & 0
\end{array}\right]
$$

and

$$
b_{k 1}=-b_{k 3}, \quad b_{k 2}=-b_{k 4} \quad(k=3,4) .
$$

By a suitable change of basis, we may also assume that $b_{33}$ is real and $b_{43}$ is purely imaginary. Applying Proposition 4.1 to $\left\langle A, B_{3}\right\rangle$ and to $\left\langle A, B_{4}\right\rangle$, we 
have

$$
b_{31} b_{33}+b_{32} b_{34}=-b_{33}^{2}-b_{34}^{2}>0,
$$

and

$$
b_{41} b_{43}+b_{42} b_{44}=-b_{43}^{2}-b_{44}^{2}>0 \text {. }
$$

So we know that every entry of $B_{3}$ and $B_{4}$ is either real or purely imaginary. Let us apply Proposition 4.1 to $\left\langle A, \xi B_{3}+\eta B_{4}\right\rangle$ with $\xi$, $\eta$ arbitrarily fixed reals. Thus the necessary and sufficient condition is that the inequality

$$
-\left(b_{33} \xi+b_{43} \eta\right)^{2}-\left(b_{34} \xi+b_{44} \eta\right)^{2}>0
$$

holds for all $(\xi, \eta) \in \mathbb{R}^{2} \backslash\{(0,0)\}$. From this, the conclusion immediately follows. As for uniform real-diagonalizability, we need only proceed in a similar way to that for the proof of Proposition 5.1.

Proposition 7.2. Let a matrix family $\left\langle A, B_{1}, \ldots, B_{n}\right\rangle(n \geq 5)$ be spanned by

$$
A=\left[\begin{array}{lll}
1 & 0 & 0 \\
0 & 0 & 0 \\
0 & 0 & 0
\end{array}\right], \quad B_{j}=\left[\begin{array}{ccc}
0 & b_{j 1} & b_{j 2} \\
b_{j 3} & 0 & 0 \\
b_{j 4} & 0 & 0
\end{array}\right] \quad(j=1, \ldots, n)
$$

where $b_{j k}(j=1, \ldots, 5 ; k=1, \ldots, 4)$ are complex constants. If $\left\langle A, B_{1}, \ldots, B_{n}\right\rangle$ is real-diagonalizable, then it is degenerate.

Proof. Assume, to the contrary, that $\left\langle A, B_{1}, \ldots, B_{n}\right\rangle$ is nondegenerate. Hence we can proceed just in the same way as in the proof of the preceding Proposition 7.1. So we may also assume

$$
B_{1}=\left[\begin{array}{lll}
0 & 1 & 0 \\
1 & 0 & 0 \\
0 & 0 & 0
\end{array}\right], \quad B_{2}=\left[\begin{array}{lll}
0 & 0 & 1 \\
0 & 0 & 0 \\
1 & 0 & 0
\end{array}\right]
$$

and

$$
b_{k 1}=-b_{k 3}, \quad b_{k 2}=-b_{k 4} \quad(k=3,4,5) .
$$

By a suitable change of basis, we may also assume that $b_{33}$ is real, $b_{43}$ is purely imaginary and

$$
b_{53}=0 \text {. }
$$

Applying Proposition 4.1 to $\left\langle A, B_{3}\right\rangle,\left\langle A, B_{4}\right\rangle$, and $\left\langle A, B_{5}\right\rangle$ we have

$$
\begin{aligned}
& b_{31} b_{33}+b_{32} b_{34}=-b_{33}{ }^{2}-b_{34}{ }^{2}>0, \\
& b_{41} b_{43}+b_{42} b_{44}=-b_{43}{ }^{2}-b_{44}{ }^{2}>0, \\
& b_{51} b_{53}+b_{52} b_{54}=-b_{54}{ }^{2}>0 .
\end{aligned}
$$


Thus, multiplying $B_{3}$ and $B_{5}$ by suitable real scalars, we may assume

$$
B_{3}=\left[\begin{array}{ccc}
0 & -\alpha & -i \\
\alpha & 0 & 0 \\
i & 0 & 0
\end{array}\right], \quad B_{5}=\left[\begin{array}{ccc}
0 & 0 & -i \\
0 & 0 & 0 \\
i & 0 & 0
\end{array}\right] \quad(\alpha: \text { real })
$$

Since $\left\langle A, B_{1}, \ldots, B_{n}\right\rangle$ is nondegenerate, we have $B_{3}-B_{5} \neq 0$, that is $\alpha \neq 0$. Now, $B_{3}-B_{5}$ must have imaginary eigenvalues $\pm i \alpha$. We are thus led to a contradiction.

As already proved in Proposition 5.3, the family of the form:

$$
\left\langle\left[\begin{array}{lll}
1 & 0 & 0 \\
0 & 0 & 0 \\
0 & 0 & 0
\end{array}\right],\left[\begin{array}{rrr}
0 & * & * \\
* & 1 & 0 \\
* & 0 & -1
\end{array}\right],\left[\begin{array}{rrr}
0 & * & * \\
* & 0 & 0 \\
* & 0 & 0
\end{array}\right],\left[\begin{array}{lll}
0 & * & * \\
* & 0 & 0 \\
* & 0 & 0
\end{array}\right], \ldots\right\rangle
$$

is equivalent to a hermitian family. Similarly, by virtue of Proposition 6.3, the family of the form:

$$
\left\langle\left[\begin{array}{lll}
1 & 0 & 0 \\
0 & 0 & 0 \\
0 & 0 & 0
\end{array}\right],\left[\begin{array}{rrr}
0 & * & * \\
* & 1 & 0 \\
* & 0 & -1
\end{array}\right],\left[\begin{array}{lll}
0 & * & * \\
* & 0 & 1 \\
* & 1 & 0
\end{array}\right],\left[\begin{array}{lll}
0 & * & * \\
* & 0 & 0 \\
* & 0 & 0
\end{array}\right], \ldots\right\rangle
$$

is also equivalent to a hermitian family. Let us now prove the family of the form:

$$
\left.\left\langle\left[\begin{array}{lll}
1 & 0 & 0 \\
0 & 0 & 0 \\
0 & 0 & 0
\end{array}\right],\left[\begin{array}{rrr}
0 & * & * \\
* & 1 & 0 \\
* & 0 & -1
\end{array}\right],\left[\begin{array}{rrr}
0 & * & * \\
* & 0 & 1 \\
* & 1 & 0
\end{array}\right],\left[\begin{array}{rrr}
0 & * & * \\
* & 0 & -i \\
* & i & 0
\end{array}\right],\left[\begin{array}{rrr}
0 & * & * \\
* & 0 & 0 \\
* & 0 & 0
\end{array}\right], \ldots\right\rangle\right\rangle .
$$

is also equivalent to a hermitian family.

Proposition 7.3. Let a nondegenerate matrix family $\left\langle A, \mathbb{B}_{1}, \mathbb{B}_{2}, B_{3}, C_{1}, \ldots\right.$, $C_{n}>(n \geq 1)$ be spanned by

$$
\begin{aligned}
A & =\left[\begin{array}{lll}
1 & 0 & 0 \\
0 & 0 & 0 \\
0 & 0 & 0
\end{array}\right], \\
B_{1} & =\left[\begin{array}{ccc}
0 & \beta_{11}+i \tilde{\beta}_{11} & \beta_{12}+i \tilde{\beta}_{12} \\
\beta_{13}+i \tilde{\beta}_{13} & 1 & 0 \\
\beta_{14}+i \tilde{\beta}_{14} & 0 & -1
\end{array}\right], \\
B_{2} & =\left[\begin{array}{ccc}
0 & \beta_{21}+i \tilde{\beta}_{21} & \beta_{22}+i \tilde{\beta}_{22} \\
\beta_{23}+i \tilde{\beta}_{23} & 0 & 1 \\
\beta_{24}+i \tilde{\beta}_{24} & 1 & 0
\end{array}\right]
\end{aligned}
$$




$$
\begin{aligned}
B_{3} & =\left[\begin{array}{ccc}
0 & \beta_{31}+i \tilde{\beta}_{31} & \beta_{32}+i \tilde{\beta}_{32} \\
\beta_{33}+i \tilde{\beta}_{33} & 0 & -i \\
\beta_{34}+i \tilde{\beta}_{34} & i & 0
\end{array}\right], \\
C_{j} & =\left[\begin{array}{ccc}
0 & \gamma_{j 1}+i \tilde{\gamma}_{j 1} & \gamma_{j 2}+i \tilde{\gamma}_{j 2} \\
\gamma_{j 3}+i \tilde{\gamma}_{j 3} & 0 & 0 \\
\gamma_{j 4}+i \tilde{\gamma}_{j 4} & 0 & 0
\end{array}\right],
\end{aligned}
$$

where $\beta_{j k}, \tilde{\beta}_{j k}, \gamma_{j k}, \tilde{\gamma}_{j k}$ are arbitrary real constants. If $\left\langle A, B_{1}, B_{2}, B_{3}, C_{1}, \ldots, C_{n}\right\rangle$ is real-diagonalizable, then it is equivalent to a hermitian family.

Remark. The proof is similar essentially to that of Proposition 6.3 rather than to that of Proposition 6.4.

Proof. Consider

$$
\begin{aligned}
\left(\eta_{1}{ }^{2}\right. & \left.+\eta_{2}{ }^{2}-\eta_{3}{ }^{2}\right) B_{1}+2 \eta_{1} \eta_{3} B_{2}+2 \eta_{2} \eta_{3} B_{3}+\sum_{k=1}^{\eta} \zeta_{k} C_{k} \\
& \equiv\left[\begin{array}{ccc}
0 & \rho_{1}(\eta, \zeta)+i \tilde{\rho}_{1}(\eta, \zeta) & \rho_{2}(\eta, \zeta)+i \tilde{\rho}_{2}(\eta, \zeta) \\
\rho_{3}(\eta, \zeta)+i \tilde{\rho}_{3}(\eta, \zeta) & \eta_{1}{ }^{2}+\eta_{2}{ }^{2}-\eta_{3}{ }^{2} & 2 \eta_{3}\left(\eta_{1}-i \eta_{2}\right) \\
\rho_{4}(\eta, \zeta)+i \tilde{\rho}_{4}(\eta, \zeta) & 2 \eta_{3}\left(\eta_{1}+i \eta_{2}\right) & -\eta_{1}{ }^{2}-\eta_{2}{ }^{2}+\eta_{3}{ }^{2}
\end{array}\right]
\end{aligned}
$$

with any fixed $(\eta, \zeta)=\left(\eta_{1}, \eta_{2}, \eta_{3}, \zeta_{1}, \ldots, \zeta_{n}\right) \in \mathbb{R}^{3+n} \backslash\{(0,0, \ldots, 0)\}$. Here

$$
\begin{aligned}
& \rho_{j}(\eta) \equiv \beta_{1 j}\left(\eta_{1}{ }^{2}+\eta_{2}{ }^{2}-\eta_{3}{ }^{2}\right)+2 \beta_{2 j} \eta_{1} \eta_{3}+2 \beta_{3 j} \eta_{2} \eta_{3}+\sum_{k=1}^{n} \gamma_{k j} \zeta_{k}, \\
& \tilde{\rho}_{j}(\eta) \equiv \tilde{\beta}_{1 j}\left(\eta_{1}{ }^{2}+\eta_{2}{ }^{2}-\eta_{3}{ }^{2}\right)+2 \tilde{\beta}_{2 j} \eta_{1} \eta_{3}+2 \tilde{\beta}_{3 j} \eta_{2} \eta_{3}+\sum_{k=1}^{n} \tilde{\gamma}_{k j} \zeta_{k} .
\end{aligned}
$$

for $j=1,2,3,4$. Note that

$$
\begin{aligned}
& \left\langle A,\left(\eta_{1}{ }^{2}+\eta_{2}{ }^{2}-\eta_{3}{ }^{2}\right) B_{1}+2 \eta_{1} \eta_{3} B_{2}+2 \eta_{2} \eta_{3} B_{3}+\sum_{k=1}^{n} \zeta_{k} C_{k}\right\rangle \\
& \subset\left\langle A, B_{1}, B_{2}, B_{3}, C_{1}, \ldots, C_{n}\right\rangle
\end{aligned}
$$

is clearly real-diagonalizable. We define a unitary matrix $U(\eta)$ by

$$
U(\eta) \equiv\left[\begin{array}{ccc}
1 & 0 & 0 \\
0 & \left(\eta_{1}-i \eta_{2}\right) /\|\eta\| & -\eta_{3} /\|\eta\| \\
0 & \eta_{3} /\|\eta\| & \left(\eta_{1}+i \eta_{2}\right) /\|\eta\|
\end{array}\right]
$$

where

$$
\|\eta\|=\left(\eta_{1}{ }^{2}+\eta_{2}{ }^{2}+\eta_{3}{ }^{2}\right)^{1 / 2} .
$$

By the similarity transformation with this $U(\eta)$,

$$
\left\langle A,\left(\eta_{1}{ }^{2}+\eta_{2}{ }^{2}-\eta_{3}{ }^{2}\right) B_{1}+2 \eta_{1} \eta_{3} B_{2}+2 \eta_{2} \eta_{3} B_{3}+\sum_{k=1}^{n} \zeta_{k} C_{k}\right\rangle
$$

is equivalent to 
$\left\langle\left[\begin{array}{lll}1 & 0 & 0 \\ 0 & 0 & 0 \\ 0 & 0 & 0\end{array}\right],\|\eta\|^{-1} \cdot\left[\begin{array}{ccc}0 & \sigma_{1}(\eta, \zeta)+i \tilde{\sigma}_{1}(\eta, \zeta) & \sigma_{2}(\eta, \zeta)+i \tilde{\sigma}_{2}(\eta, \zeta) \\ \sigma_{3}(\eta, \zeta)+i \tilde{\sigma}_{3}(\eta, \zeta) & \|\eta\|^{3} & 0 \\ \sigma_{4}(\eta, \zeta)+i \tilde{\sigma}_{4}(\eta, \zeta) & 0 & -\|\eta\|^{3}\end{array}\right]\right\rangle$

where

$$
\begin{aligned}
& \sigma_{1}(\eta) \equiv\left(\eta_{1}{ }^{2}+\eta_{2}{ }^{2}-\eta_{3}{ }^{2}\right)\left(\beta_{11} \eta_{1}+\tilde{\beta}_{11} \eta_{2}+\beta_{12} \eta_{3}\right) \\
& +2 \eta_{1} \eta_{3}\left(\beta_{21} \eta_{1}+\tilde{\beta}_{21} \eta_{2}+\beta_{22} \eta_{3}\right)+2 \eta_{2} \eta_{3}\left(\beta_{31} \eta_{1}+\tilde{\beta}_{31} \eta_{2}+\beta_{32} \eta_{3}\right) \\
& +\sum_{k=1}^{n}\left(\gamma_{k 1} \eta_{1}+\tilde{\gamma}_{k 1} \eta_{2}+\gamma_{k 2} \eta_{3}\right) \zeta_{k} \\
& \tilde{\sigma}_{1}(\eta) \equiv\left(\eta_{1}{ }^{2}+\eta_{2}{ }^{2}-\eta_{3}{ }^{2}\right)\left(\tilde{\beta}_{11} \eta_{1}-\beta_{11} \eta_{2}+\tilde{\beta}_{12} \eta_{3}\right) \\
& +2 \eta_{1} \eta_{3}\left(\tilde{\beta}_{21} \eta_{1}-\beta_{21} \eta_{2}+\tilde{\beta}_{22} \eta_{3}\right)+2 \eta_{2} \eta_{3}\left(\tilde{\beta}_{31} \eta_{1}-\beta_{31} \eta_{2}+\tilde{\beta}_{32} \eta_{3}\right) \\
& +\sum_{k=1}^{n}\left(\tilde{\gamma}_{k 1} \eta_{1}-\gamma_{k 1} \eta_{2}+\tilde{\gamma}_{k 2} \eta_{3}\right) \zeta_{k}, \\
& \sigma_{2}(\eta) \equiv\left(\eta_{1}{ }^{2}+\eta_{2}{ }^{2}-\eta_{3}{ }^{2}\right)\left(\beta_{12} \eta_{1}-\tilde{\beta}_{12} \eta_{2}-\beta_{11} \eta_{3}\right) \\
& +2 \eta_{1} \eta_{3}\left(\beta_{22} \eta_{1}-\tilde{\beta}_{22} \eta_{2}-\beta_{21} \eta_{3}\right)+2 \eta_{2} \eta_{3}\left(\beta_{32} \eta_{1}-\tilde{\beta}_{32} \eta_{2}-\beta_{31} \eta_{3}\right) \\
& +\sum_{k=1}^{n}\left(\gamma_{k 2} \eta_{1}-\tilde{\gamma}_{k 2} \eta_{2}-\gamma_{k 1} \eta_{3}\right) \zeta_{k} \\
& \tilde{\sigma}_{2}(\eta) \equiv\left(\eta_{1}{ }^{2}+\eta_{2}{ }^{2}-\eta_{3}{ }^{2}\right)\left(\tilde{\beta}_{12} \eta_{1}+\beta_{12} \eta_{2}-\tilde{\beta}_{11} \eta_{3}\right) \\
& +2 \eta_{1} \eta_{3}\left(\tilde{\beta}_{22} \eta_{1}+\beta_{22} \eta_{2}-\tilde{\beta}_{21} \eta_{3}\right)+2 \eta_{2} \eta_{3}\left(\tilde{\beta}_{32} \eta_{1}+\beta_{32} \eta_{2}-\tilde{\beta}_{31} \eta_{3}\right) \\
& +\sum_{k=1}^{n}\left(\tilde{\gamma}_{k 2} \eta_{1}+\gamma_{k 2} \eta_{2}-\tilde{\gamma}_{k 1} \eta_{3}\right) \zeta_{k} \\
& \sigma_{3}(\eta) \equiv\left(\eta_{1}{ }^{2}+\eta_{2}{ }^{2}-\eta_{3}{ }^{2}\right)\left(\beta_{13} \eta_{1}-\tilde{\beta}_{13} \eta_{2}+\beta_{14} \eta_{3}\right) \\
& +2 \eta_{1} \eta_{3}\left(\beta_{23} \eta_{1}-\tilde{\beta}_{23} \eta_{2}+\beta_{24} \eta_{3}\right)+2 \eta_{2} \eta_{3}\left(\beta_{33} \eta_{1}-\tilde{\beta}_{33} \eta_{2}+\beta_{34} \eta_{3}\right) \\
& +\sum_{k=1}^{n}\left(\gamma_{k 3} \eta_{1}-\tilde{\gamma}_{k 3} \eta_{2}+\gamma_{k 4} \eta_{3}\right) \zeta_{k}, \\
& \tilde{\sigma}_{3}(\eta) \equiv\left(\eta_{1}{ }^{2}+\eta_{2}{ }^{2}-\eta_{3}{ }^{2}\right)\left(\tilde{\beta}_{13} \eta_{1}+\beta_{13} \eta_{2}+\tilde{\beta}_{14} \eta_{3}\right) \\
& +2 \eta_{1} \eta_{3}\left(\tilde{\beta}_{23} \eta_{1}+\beta_{23} \eta_{2}+\tilde{\beta}_{24} \eta_{3}\right)+2 \eta_{2} \eta_{3}\left(\tilde{\beta}_{33} \eta_{1}+\beta_{33} \eta_{2}+\tilde{\beta}_{34} \eta_{3}\right) \\
& +\sum_{k=1}^{n}\left(\tilde{\gamma}_{k 3} \eta_{1}+\gamma_{k 3} \eta_{2}+\tilde{\gamma}_{k 4} \eta_{3}\right) \zeta_{k}, \\
& \sigma_{4}(\eta) \equiv\left(\eta_{1}{ }^{2}+\eta_{2}{ }^{2}-\eta_{3}{ }^{2}\right)\left(\beta_{14} \eta_{1}+\tilde{\beta}_{14} \eta_{2}-\beta_{13} \eta_{3}\right) \\
& +2 \eta_{1} \eta_{3}\left(\beta_{24} \eta_{1}+\tilde{\beta}_{24} \eta_{2}-\beta_{23} \eta_{3}\right)+2 \eta_{2} \eta_{3}\left(\beta_{34} \eta_{1}+\tilde{\beta}_{34} \eta_{2}-\beta_{33} \eta_{3}\right) \\
& +\sum_{k=1}^{n}\left(\gamma_{k 4} \eta_{1}+\tilde{\gamma}_{k 4} \eta_{2}-\gamma_{k 4} \eta_{3}\right) \zeta_{k}, \\
& \tilde{\sigma}_{4}(\eta) \equiv\left(\eta_{1}{ }^{2}+\eta_{2}{ }^{2}-\eta_{3}{ }^{2}\right)\left(\tilde{\beta}_{14} \eta_{1}-\beta_{14} \eta_{2}-\tilde{\beta}_{13} \eta_{3}\right) \\
& +2 \eta_{1} \eta_{3}\left(\tilde{\beta}_{24} \eta_{1}-\beta_{24} \eta_{2}-\tilde{\beta}_{23} \eta_{3}\right)+2 \eta_{2} \eta_{3}\left(\tilde{\beta}_{34} \eta_{1}-\beta_{34} \eta_{2}-\tilde{\beta}_{33} \eta_{3}\right) \\
& +\sum_{k=1}^{n}\left(\tilde{\gamma}_{k 4} \eta_{1}-\gamma_{k 4} \eta_{2}-\tilde{\gamma}_{k 4} \eta_{3}\right) \zeta_{k} \text {. }
\end{aligned}
$$


Note that all of $\sigma_{k}(\eta, \zeta)$ and $\tilde{\sigma}_{k}(\eta, \zeta)(k=1, \ldots, 4)$ are linear polynomials with respect to $\zeta=\left(\zeta_{1}, \ldots, \zeta_{n}\right)$ and each of their coefficients of $\zeta_{k}(k=1, \ldots, n)$ is a linear polynomial in $\eta=\left(\eta_{1}, \eta_{2}, \eta_{3}\right)$. Let us apply Proposition 4.3 and Lemma 3.2 to

$$
\sigma_{1}(\eta, \zeta)+i \tilde{\sigma}_{1}(\eta, \zeta)
$$

and

$$
\sigma_{3}(\eta, \zeta)+i \tilde{\sigma}_{3}(\eta, \zeta)
$$

Thus we have

$$
\begin{aligned}
& \operatorname{sgn} \sigma_{1}(\eta, \zeta) \equiv \operatorname{sgn} \sigma_{3}(\eta, \zeta) \\
& \operatorname{sgn} \tilde{\sigma}_{1}(\eta, \zeta) \equiv-\operatorname{sgn} \tilde{\sigma}_{3}(\eta, \zeta)
\end{aligned}
$$

Recall that $\sigma(\eta, \zeta)$ and $\tilde{\sigma}(\eta, \zeta)$ are linear with respect to $\zeta$ and their coefficients of $\zeta$ are linear polynomials in $\eta$. Therefore the same reasoning is valid as for Proposition 6.3. And we obtain

$$
\sigma_{3}(\eta, \zeta) \equiv \alpha \sigma_{1}(\eta, \zeta), \quad \tilde{\sigma}_{3}(\eta, \zeta) \equiv-\alpha \tilde{\sigma}_{1}(\eta, \zeta)
$$

for some $\alpha>0$. As a consequence, we have

$$
\beta_{j 3}=\alpha \beta_{j 1}, \quad \beta_{j 4}=\alpha \beta_{j 2}, \quad \tilde{\beta}_{j 3}=-\alpha \tilde{\beta}_{j 1}, \quad \tilde{\beta}_{j 4}=-\alpha \tilde{\beta}_{j 2},
$$

for $j=1,2,3$ and

$$
\gamma_{k 3}=\alpha \gamma_{k 1}, \quad \gamma_{k 4}=\alpha \gamma_{k 2}, \quad \tilde{\gamma}_{k 3}=-\alpha \tilde{\gamma}_{k 1}, \quad \tilde{\gamma}_{k 4}=-\alpha \tilde{\gamma}_{k 2}
$$

for $k=1, \ldots, n$. Therefore $A, B_{1}, B_{2}, B_{3}, C_{1}, \ldots, C_{n}$ can be transformed to hermitian matrices by the similarity transformation with

$$
T=\left[\begin{array}{ccc}
\sqrt{\alpha} & 0 & 0 \\
0 & 1 & 0 \\
0 & 0 & 1
\end{array}\right]
$$

The proof is completed.

Finally, let us turn our attention to non-uniformly real-diagonalizable families. We need only consider

$$
\left\langle\left[\begin{array}{lll}
1 & 0 & 0 \\
0 & 0 & 0 \\
0 & 0 & 0
\end{array}\right],\left[\begin{array}{lll}
0 & 1 & 0 \\
1 & 0 & 1 \\
0 & 0 & 0
\end{array}\right],\left[\begin{array}{lll}
0 & * & * \\
* & 0 & * \\
* & 0 & 0
\end{array}\right],\left[\begin{array}{lll}
0 & * & * \\
* & 0 & * \\
* & 0 & 0
\end{array}\right], \ldots\right\rangle
$$

or

$$
\left\langle\left[\begin{array}{lll}
1 & 0 & 0 \\
0 & 0 & 0 \\
0 & 0 & 0
\end{array}\right],\left[\begin{array}{lll}
0 & 1 & 0 \\
1 & 0 & 1 \\
0 & 0 & 0
\end{array}\right],\left[\begin{array}{rrr}
0 & * & * \\
* & 1 & * \\
* & 0 & -1
\end{array}\right],\left[\begin{array}{lll}
0 & * & * \\
* & 0 & * \\
* & 0 & 0
\end{array}\right], \ldots\right\rangle .
$$


Here, we can ignore the first one because we have discussed it in Proposition 6.5. So let us consider the second one.

Proposition 7.4. Let a matrix family $\left\langle A, B, C, D_{1}, \ldots, \mathbb{D}_{n}\right\rangle(n \geq 2)$ be spanned by

$$
\begin{array}{ll}
A & =\left[\begin{array}{lll}
1 & 0 & 0 \\
0 & 0 & 0 \\
0 & 0 & 0
\end{array}\right], \quad B=\left[\begin{array}{lll}
0 & 1 & 0 \\
1 & 0 & 1 \\
0 & 0 & 0
\end{array}\right], \quad C=\left[\begin{array}{ccc}
0 & \gamma_{1}+i \tilde{\gamma}_{1} & \gamma_{2}+i \tilde{\gamma}_{2} \\
\gamma_{3}+i \tilde{\gamma}_{3} & 1 & \gamma_{5}+i \tilde{\gamma}_{5} \\
\gamma_{4}+i \tilde{\gamma}_{4} & 0 & -1
\end{array}\right], \\
\mathbb{D}_{k} & =\left[\begin{array}{ccc}
0 & \delta_{k 1}+i \tilde{\delta}_{k 1} & \delta_{k 2}+i \tilde{\delta}_{k 2} \\
\delta_{k 3}+i \tilde{\delta}_{k 3} & 0 & \delta_{k 5}+i \tilde{\delta}_{k 5} \\
\delta_{k 4}+i \tilde{\delta}_{k 4} & 0 & 0
\end{array}\right]
\end{array}
$$

where $\gamma_{j}, \tilde{\gamma}_{j}, \delta_{j}, \tilde{\delta}_{j}(k=1, \ldots, n ; j=1, \ldots, 5)$ are real constants. If the family $\left\langle A, B, C, D_{1}, \ldots, D_{n}\right\rangle$ is real-diagonalizable, then it is degenerate.

Proof. Apply Proposition 6.5 to $\left\langle A, B, D_{1}, \ldots, D_{n}\right\rangle$.

The results obtained in this section are summarized as follows.

Theorem 7.5. Let $\left\langle A_{1}, \ldots, A_{n}\right\rangle$ be a nondegenerate $3 \times 3$ matrix family with multiple eigenvalues. Then the following 1) and 2) hold.

1) Suppose $n \geq 6$ and that $\left\langle A_{1}, \ldots, A_{n}\right\rangle$ is real-diagonalizable. Then it is equivalent to a hermitian family.

2) Suppose $n=5$ and that $\left\langle A_{1}, \ldots, A_{n}\right\rangle$ is real-diagonalizable. Then it is uniformly real-diagonalizable. In this case, it is equivalent either to

$$
\left\langle\left[\begin{array}{lll}
1 & 0 & 0 \\
0 & 0 & 0 \\
0 & 0 & 0
\end{array}\right],\left[\begin{array}{lll}
0 & 1 & 0 \\
1 & 0 & 0 \\
0 & 0 & 0
\end{array}\right],\left[\begin{array}{lll}
0 & 0 & 1 \\
0 & 0 & 0 \\
1 & 0 & 0
\end{array}\right],\left[\begin{array}{rrr}
0 & -\alpha & -i \\
\alpha & 0 & 0 \\
i & 0 & 0
\end{array}\right],\left[\begin{array}{rrr}
0 & -i & \alpha \\
i & 0 & 0 \\
-\alpha & 0 & 0
\end{array}\right]\right\rangle
$$

with a real constant $\alpha$ satisfying $0<\alpha<1$, or to a hermitian family.

\section{\$\&. Summary}

As a summary of this paper, we reprint here Theorem 7.5, 6.8, 5.15, 4.7, in this order. However, we rename them for the present section to look natural as a classification table.

Theorem 8.1 . Let $\left\langle A_{1}, \ldots, A_{n}\right\rangle$ be a nondegenerate $3 \times 3$ matrix family with multiple eigenvalues. Then the following 1) and 2) hold.

1) Suppose $n \geq 6$ and that $\left\langle A_{1}, \ldots, A_{n}\right\rangle$ is real-diagonalizable. Then it is equivalent to a hermitian family.

2) Suppose $n=5$ and that $\left\langle A_{1}, \ldots, A_{n}\right\rangle$ is real-diagonalizable. Then it is 
uniformly real-diagonalizable. In this case, it is equivalent either to

$$
\left\langle\left[\begin{array}{lll}
1 & 0 & 0 \\
0 & 0 & 0 \\
0 & 0 & 0
\end{array}\right],\left[\begin{array}{lll}
0 & 1 & 0 \\
1 & 0 & 0 \\
0 & 0 & 0
\end{array}\right],\left[\begin{array}{lll}
0 & 0 & 1 \\
0 & 0 & 0 \\
1 & 0 & 0
\end{array}\right],\left[\begin{array}{rrr}
0 & -\alpha & -i \\
\alpha & 0 & 0 \\
i & 0 & 0
\end{array}\right],\left[\begin{array}{rrr}
0 & -i & \alpha \\
i & 0 & 0 \\
-\alpha & 0 & 0
\end{array}\right]\right\rangle
$$

with a real constant $\alpha$ satisfying $0<\alpha<1$, or to a hermitian family.

Theorem 8.2. Let $\left\langle A_{1}, A_{2}, A_{3}, A_{4}\right\rangle$ be a nondegenerate $3 \times 3$ matrix family. Then the following 1) and 2) hold.

1) Suppose that the family $\left\langle A_{1}, A_{2}, A_{3}, A_{4}\right\rangle$ has multiple eigenvalues and is not equivalent to any hermitian family. Then it is uniformly real-diagonalizable if and only if it is equivalent to either

$$
\left\langle\left[\begin{array}{lll}
1 & 0 & 0 \\
0 & 0 & 0 \\
0 & 0 & 0
\end{array}\right],\left[\begin{array}{lll}
0 & 1 & 0 \\
1 & 0 & 0 \\
0 & 0 & 0
\end{array}\right],\left[\begin{array}{lll}
0 & 0 & 1 \\
0 & 0 & 0 \\
1 & 0 & 0
\end{array}\right],\left[\begin{array}{ccc}
0 & -c+\frac{1}{c} & -i\left(c+\frac{1}{c}\right) \\
c-\frac{1}{c} & 0 & 0 \\
i\left(c+\frac{1}{c}\right) & 0 & 0
\end{array}\right]\right\rangle
$$

with a complex constant $c$ satisfying $|c| \neq 0,1$, or

$\left\langle\left[\begin{array}{lll}1 & 0 & 0 \\ 0 & 0 & 0 \\ 0 & 0 & 0\end{array}\right],\left[\begin{array}{lll}0 & 1 & 0 \\ 1 & 0 & 0 \\ 0 & 0 & 0\end{array}\right],\left[\begin{array}{rrr}0 & -\alpha & 1 \\ \alpha & 0 & 0 \\ 1 & 0 & 0\end{array}\right],\left[\begin{array}{ccc}0 & -i(1+\gamma)\left(\beta+i \frac{\alpha}{\gamma}\right) \\ i & 0 & 0 \\ (1-\gamma)\left(\beta-i \frac{\alpha}{\gamma}\right) & 0 & 0\end{array}\right]\right\rangle$

where $0<\alpha<1, \beta \neq 0, \gamma \neq 0$ are real constants satisfying $\beta^{2} \gamma^{2}<1-\alpha^{2}$, or

$$
\left\langle\left[\begin{array}{lll}
1 & 0 & 0 \\
0 & 0 & 0 \\
0 & 0 & 0
\end{array}\right],\left[\begin{array}{lll}
0 & 1 & 0 \\
1 & 0 & 0 \\
0 & 0 & 0
\end{array}\right],\left[\begin{array}{ccc}
0 & -\alpha & 1 \\
\alpha & 0 & 0 \\
1 & 0 & 0
\end{array}\right],\left[\begin{array}{ccc}
0 & -i & i(\alpha+\gamma) \\
i & 0 & 0 \\
i(\alpha-\gamma) & 0 & 0
\end{array}\right]\right\rangle
$$

where $0<\alpha<1$ and $\gamma$ are real constants, or

$$
\left\langle\left[\begin{array}{lll}
1 & 0 & 0 \\
0 & 0 & 0 \\
0 & 0 & 0
\end{array}\right],\left[\begin{array}{lll}
0 & 1 & 0 \\
1 & 0 & 0 \\
0 & 0 & 0
\end{array}\right],\left[\begin{array}{ccc}
0 & -\alpha & 1 \\
\alpha & 0 & 0 \\
1 & 0 & 0
\end{array}\right],\left[\begin{array}{ccc}
0 & -i & (i \alpha+\gamma) \\
i & 0 & 0 \\
(i \alpha-\gamma) & 0 & 0
\end{array}\right]\right\rangle
$$

where $\alpha$ and $\gamma$ are real constants satisfying $0<\alpha<1$ and $\alpha^{2}+\gamma^{2}<1$.

2) The family $\left\langle A_{1}, A_{2}, A_{3}, A_{4}\right\rangle$ is non-uniformly real-diagonalizable (necessarily with multiple eigenvalues) if and only if it is equivalent to 


$$
\left\langle\left[\begin{array}{lll}
1 & 0 & 0 \\
0 & 0 & 0 \\
0 & 0 & 0
\end{array}\right],\left[\begin{array}{lll}
0 & 1 & 0 \\
1 & 0 & 1 \\
0 & 0 & 0
\end{array}\right],\left[\begin{array}{ccc}
0 & \beta & -\gamma \\
\beta(1-\alpha) & 1 & 0 \\
-2 \alpha & 0 & -1
\end{array}\right],\left[\begin{array}{ccc}
0 & -i & \delta+\frac{i}{2} \beta \\
i & 0 & i \\
0 & 0 & 0
\end{array}\right]\right\rangle
$$

where the real constants $\alpha, \beta, \gamma$ and $\delta$ satisfy

$$
0<\alpha<1, \quad \gamma>\frac{1}{8} \beta^{2}+\frac{1}{2} \delta^{2},
$$

or their transposes.

Theorem 8.3. Let $\left\langle A_{1}, A_{2}, A_{3}\right\rangle$ be a nondegenerate $3 \times 3$ matrix family. Then the following 1) and 2) hold.

1) Suppose that the family $\left\langle A_{1}, A_{2}, A_{3}\right\rangle$ has multiple eigenvalues and is not equivalent to any hermitian family. Then it is uniformly real-diagonalizable if and only if it is equivalent to either

$$
\left\langle\left[\begin{array}{lll}
1 & 0 & 0 \\
0 & 0 & 0 \\
0 & 0 & 0
\end{array}\right],\left[\begin{array}{lll}
0 & 1 & 0 \\
1 & 0 & 0 \\
0 & 0 & 0
\end{array}\right],\left[\begin{array}{ccc}
0 & -c+\frac{1}{c} & c+\frac{1}{c} \\
c-\frac{1}{c} & 0 & 0 \\
c+\frac{1}{c} & 0 & 0
\end{array}\right]\right\rangle
$$

where $c$ is an arbitrary complex constant satisfying $|c| \neq 0,1$, or

$$
\left\langle\left[\begin{array}{lll}
1 & 0 & 0 \\
0 & 0 & 0 \\
0 & 0 & 0
\end{array}\right],\left[\begin{array}{lll}
0 & 1 & 0 \\
1 & 0 & 0 \\
0 & 0 & 0
\end{array}\right],\left[\begin{array}{rrr}
0 & -i & -i \\
i & 0 & 0 \\
0 & 0 & 0
\end{array}\right]\right\rangle
$$

or its transpose

$$
\left\langle\left[\begin{array}{lll}
1 & 0 & 0 \\
0 & 0 & 0 \\
0 & 0 & 0
\end{array}\right],\left[\begin{array}{lll}
0 & 1 & 0 \\
1 & 0 & 0 \\
0 & 0 & 0
\end{array}\right],\left[\begin{array}{rrr}
0 & -i & 0 \\
i & 0 & 0 \\
i & 0 & 0
\end{array}\right]\right\rangle
$$

or

$$
\left\langle\left[\begin{array}{lll}
1 & 0 & 0 \\
0 & 0 & 0 \\
0 & 0 & 0
\end{array}\right],\left[\begin{array}{ccc}
0 & \alpha & -i(\alpha-2 \gamma) \delta \\
\alpha & 1 & 0 \\
i\left(\alpha-2 \gamma^{\prime}\right) \delta & 0 & -1
\end{array}\right],\left[\begin{array}{ccc}
0 & \beta-i \gamma \delta & \gamma-i \beta \delta \\
\beta^{\prime}+i \gamma^{\prime} \delta & 0 & 1 \\
\gamma^{\prime}+i \beta^{\prime} \delta & 1 & 0
\end{array}\right]\right\rangle
$$

where the real constants $\alpha, \beta, \beta^{\prime}, \gamma, \gamma^{\prime}$ and $\delta$ satisfy

$$
\alpha>0, \quad \gamma>\frac{1}{2}\left(\alpha+\frac{\beta^{2}}{\alpha}\right), \quad \gamma^{\prime}>\frac{1}{2}\left(\alpha+\frac{\beta^{\prime 2}}{\alpha}\right), \quad\left|\beta-\beta^{\prime}\right|+\left|\gamma-\gamma^{\prime}\right|>0 .
$$


2) The family $\left\langle A_{1}, A_{2}, A_{3}\right\rangle$ is non-uniformly real-diagonalizable (necessarily having multiple eigenvalues) if and only if $\left\langle A_{1}, A_{2}, A_{3}\right\rangle$ is equivalent to

$$
\left\langle\left[\begin{array}{lll}
1 & 0 & 0 \\
0 & 0 & 0 \\
0 & 0 & 0
\end{array}\right],\left[\begin{array}{lll}
0 & 1 & 0 \\
1 & 0 & 1 \\
0 & 0 & 0
\end{array}\right],\left[\begin{array}{ccc}
0 & -i & c_{1} \\
i & 0 & c_{2} \\
0 & 0 & 0
\end{array}\right]\right\rangle
$$

where $c_{1}, c_{2}$ are arbitrary complex constants, or

$$
\left\langle\left[\begin{array}{lll}
1 & 0 & 0 \\
0 & 0 & 0 \\
0 & 0 & 0
\end{array}\right],\left[\begin{array}{lll}
0 & 1 & 0 \\
1 & 0 & 1 \\
0 & 0 & 0
\end{array}\right],\left[\begin{array}{ccc}
0 & \beta-i \delta & -\gamma+\frac{i}{2} \beta \delta \\
\beta(1-\alpha)+i \delta & 1 & i \delta \\
-2 \alpha & 0 & -1
\end{array}\right]\right\rangle
$$

where the real constants $\alpha, \beta, \gamma$ and $\delta$ satisfy

$$
0<\alpha<1, \quad \gamma>\frac{1}{8}\left(\beta^{2}-4 \delta^{2}\right)
$$

or their transposes.

Theorem 8.4. Let $\left\langle A_{1}, A_{2}\right\rangle$ be a nondegenerate $3 \times 3$ matrix family. Then the following holds.

1) Suppose that the family $\left\langle A_{1}, A_{2}\right\rangle$ has multiple eigenvalues and is uniformly real-diagonalizable. Then $\left\langle A_{1}, A_{2}\right\rangle$ is equivalent to a hermitian family.

2) The family $\left\langle A_{1}, A_{2}\right\rangle$ is non-uniformly real-diagonalizable (consequently, it must have multiple eigenvalues) if and only if $\left\langle A_{1}, A_{2}\right\rangle$ is equivalent to either

$$
\left\langle\left[\begin{array}{lll}
1 & 0 & 0 \\
0 & 0 & 0 \\
0 & 0 & 0
\end{array}\right],\left[\begin{array}{lll}
0 & 1 & 0 \\
1 & 0 & 1 \\
0 & 0 & 0
\end{array}\right]\right\rangle
$$

or its transpose.

\section{Acknowledgments}

The author would like to express his sincere gratitude to Professor Masaya Yamaguti of Ryukoku University and Professor Takaaki Nishida of Kyoto University for their encouragement to publish the present paper.

\section{References}

[1] Brieskorn, E. and Knörrer, H., Plane Algebraic Curves, Birkhäuser, 1986.

[2] Friedland, S., Robbin, J. M. and Sylvester, J. H., On the crossing rule, Comm. Pure Appl. Math., 37 (1984), 19-37.

[3] Gårding, L., Linear hyperbolic partial differential equations with constant coefficients, Acta Math., 85 (1951), 1-62. 
[4] Kasahara, K. and Yamaguti, M., Strongly hyperbolic systems of linear partial equations with constant coefficients, Mem. Coll. Sci. Univ. Kyoto, Ser. A, 33 (1960), 1-23.

[5] Lax, P. D., Differential equations, difference equations and matrix theory, Comm. Pure Appl. Math., $\mathbb{1} \mathbb{1}$ (1958), 175-194.

[6] Oshime, Y., Canonical forms of $3 \times 3$ strongly hyperbolic systems with real constant coefficients, J. Math. Kyoto Univ., 31 (1991), 937-982.

[7] — On the canonical forms of $3 \times 3$ nondiagonalizable hyperbolic systems with real constant coefficients, J. Math. Kyoto Univ., 31 (1991), 983-1021.

[8] Strang, G., On strong hyperbolicity, J. Math. Kyoto Univ., 6 (1967), 397-417.

[9] Yamaguti, M. and Kasahara, K, Sur le système hyperbolique à coefficients constants, Proc. Japan Acad., 35 (1959), 547-550.

[10] Oshime, Y., A special type of $3 \times 3$ strictly hyperbolic systems with complex constant coefficients, Sci. Eng. Rev. Doshiha Univ., 32 (1991), 239-258. 